Article

\title{
Future Projections for Wind, Wind Shear and Helicity in the Iberian Peninsula
}

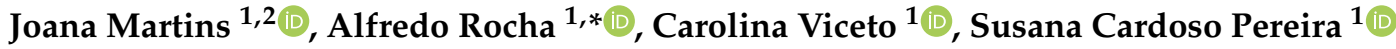 \\ and João A. Santos 2 (D) \\ 1 CESAM-Department of Physics, University of Aveiro, 3810-193 Aveiro, Portugal; \\ joanamartins@utad.pt (J.M.); carolinaviceto@ua.pt (C.V.); susana.cardoso@ua.pt (S.C.P.) \\ 2 Centre for the Research and Technology of Agro-Environmental and Biological Sciences, Universidade de \\ Trás-os-Montes e Alto Douro, UTAD, 5000-801 Vila Real, Portugal; jsantos@utad.pt \\ * Correspondence: alfredo.rocha@ua.pt
}

Received: 5 August 2020; Accepted: 11 September 2020; Published: 18 September 2020

check for updates

\begin{abstract}
Wind is among the most important climatic elements. Its characteristics are determinant for a wide range of natural processes and human activities. However, ongoing climate change is modifying these characteristics, which may have important implications. Climatic changes on wind speed and direction, wind shear intensity, and helicity, over the 21st century and for 26 cities in the Iberian Peninsula, under the Representative Concentration Pathway (RCP) 8.5 anthropogenic forcing scenario, are assessed. For this purpose, the Weather Research and Forecasting (WRF) model was used, with initial and boundary conditions being obtained from simulations with the Max Planck Institute for Meteorology Earth System Model (MPI-ESM-LR) climate model and ERA-Interim reanalysis. Quantile-quantile bias correction was applied to the simulated data prior to subsequent analysis. Overall, the results hint at a reduction in the intensity of both near-surface and $850 \mathrm{hPa}$ (approx. 5\%) wind in the future. Nevertheless, for the $300 \mathrm{hPa}$ level, a decrease in summertime wind speed is accompanied by a slight increase in the remaining months. Furthermore, significant increases in the number of occurrences of extreme wind events were also identified, mainly in northwestern Iberia. For wind shear, an intensity increase is projected throughout most of the year (approx. 5\% in the upper quantiles), mainly in southwestern Iberia. Helicity is also projected to undergo a strengthening, mostly in summer months and over southwestern Iberia, with greater emphasis on events of longer duration and intensity. This study highlights some important projected changes in the wind structure and profile under future anthropogenic forcing. This knowledge may support decisions on climate change adaptation options and risk reduction of several major sectors, such as energy and aviation, thus deserving further research.
\end{abstract}

Keywords: climate change; wind shear; helicity; jet stream; extreme events; Iberian Peninsula

\section{Introduction}

Climate change impacts on the wind can have noteworthy consequences across vast areas of the Earth. Changes in the average wind speed can influence potential evapotranspiration and, consequently, the availability of water in the soil, which can contribute to droughts [1]. Wind also plays a central role in forest fire development [2]. Prolonged wind periods in mid-latitudes can lead to sea-level rise in coastal regions [3], while prolonged periods in which a given wind direction prevails can cause changes in waves and coastal stability [4]. The structure of the vertical wind profile can also significantly affect aviation [5]. There are still other wind properties that may influence the development of storms, such as the position of the jet stream in the extratropical latitudes, which is decisive for the development of storms $[6,7]$ as well as in triggering hydrological deficits and droughts $[8,9]$. Furthermore, wind is 
today considered to be a relevant energy resource in an economy in transition from fossil energies to renewable energies [10].

Many studies have revealed changes in the intensity of the near-surface wind in several regions worldwide [11]. For instance, in Europe, there are several countries where this reduction has been reported, such as France [12], Czech Republic [13], Netherlands [14], Turkey [15], Spain, and Portugal $[10,16]$. Some studies have shown that the causes that are associated with the decrease in the intensity of surface wind may be associated with changes in air temperature [15], increased aerosol emission [11], and also changes in large-scale and regional-scale circulation patterns [17], such as in the North Atlantic Oscillation, NAO [18-20]. One of the reasons for studying climate change projections of wind speed under a future climate scenario is the exploitation of wind energy potential. According to [21], an increase in the wind energy resource in Central Europe (Baltic Sea and neighbouring countries) and a decrease in the Mediterranean region are expected to occur, mainly at the end of this century and under scenarios of strong radiative anthropogenic forcing. Moreover, an increase in intra-annual variability in the Baltic Sea, and neighbouring areas, and a decrease in the Mediterranean Basin are projected, but there are no significant changes in intra-annual variability for the rest of Europe.

It is of particular interest to study the consequences of climate change concerning the jet stream, since it dictates aviation routes and flight times, mainly in the case of the transatlantic flights. Climate models indicate an approximate $1^{\circ}$ latitude poleward shift and a slight strengthening of the jet stream by 2100 [22]. Another study [23] identifies contrasting responses, depending on the season and longitude. In winter, a slowing of the jet stream and increase in its waviness over North America were projected, whereas an increase in current intensity and a decrease in waviness can be anticipated over Europe. While the warming of the upper tropical troposphere shifts the jet stream polewards in winter, the opposite effect is found when a weakening of the polar vortex occurs. The position and intensity of the jet stream are fundamental features in the development of extratropical depressions.

However, despite the changes in the mean climate state, it is also necessary to consider changes in other statistical properties, namely in the variability and extreme events. Extreme wind intensities are a threat to the safety of human life, of maritime activities, aviation, and infrastructure integrity [24], among others. Extreme winds at the surface are strongly associated with storms [25]. An intense storm can combine winds and extreme precipitation. The lack of wind over an extended period may also be considered an extreme event, which can, as an example, lead to an excessive accumulation of urban pollution [26]. Wind extremes are often considered in the context of extreme phenomena or natural hazards, frequently associated with tropical and extratropical cyclones, storms, downbursts, and tornadoes [24].

The storm-relative helicity (SRH) is a sounding-derived parameter related to severe weather. It is commonly studied, because it assesses the potential for cyclonic rotation in the updraft of right-moving supercells (e.g., [27-29]). SRH between 0 and $3 \mathrm{~km}$ can be used to differentiate a non-tornadic storm from a tornadic one (Enhanced Fujita scale EF1 or more) [30]. However, it is impossible to distinguish weak tornadoes from non-tornadic thunderstorm events, as they present similar values. The formation of an organized convective storm system depends on the wind shear intensity. High values of wind shear can lead to long-lived storm cells and help them evolve into multi-cells or supercells [31]. It is also known that greater values of wind shear $(0-6 \mathrm{~km})$ create a higher probability of a stronger tornado [30]. With around 240 per year, tornados in some regions of Europe are relatively frequent and potentially dangerous [32,33]. In the Iberian Peninsula, Catalonia is the region where tornadoes are most frequent [34-36]. By examining tornado reports from 1950 to 2015 in Europe, it was found that they are relatively common on the western Mediterranean coastal areas [37]. Some authors have studied the environmental conditions that favour tornadic events [32,38,39]. An increase in extreme winds in tropical cyclones is also projected for the future and several regional studies support the hypothesis of increased storm risk that is associated with wind during the winter months in Europe, mainly due to the change in their trajectories $[25,40-44]$. 
It is possible to find some studies for the 21st century regarding extreme events in Portugal related to variations in temperature and precipitation [45-47]. However, there is insufficient information regarding the occurrence of extreme winds or storms, particularly their association with strong wind shear and helicity. One previous study reveals that projections for the 21st century indicate a robust increase in the wind between June and November for the tropical Atlantic Ocean and the East Pacific region. At the Caribbean Sea, Tropical North Atlantic, and Eastern Tropical Pacific, the wind shear increases between 0.5 to $1 \mathrm{~m} \mathrm{~s}^{-1}$ for each ${ }^{\circ} \mathrm{C}$ of global warming. Changes in the wind shear over the Atlantic Ocean are related to changes in the zonal wind in the upper troposphere [48]. A second study presents a preliminary comparison between the simulation for the 21st century CCSM3 (Community Climate System Model 3), following the IPCC A2 emission scenario, and the 20th-century conditions. A slight increase in CAPE (convective available potential energy) is shown for the cold season, followed by a slight decrease for the warm season, which is accompanied by a small variation in the mean wind shear. However, there is an enhancement of the atmospheric conditions that are favourable to the occurrence of severe events in most locations, resulting from increased CAPE and wind shear. Nearby regions of the Mediterranean Sea experience an increase in CAPE and more favourable conditions to these events [49], while regions across northern Europe undergo little change [50].

However, Global Climate Models (GCMs), when considering their current spatial resolution, are unable to properly resolve such small-scale mechanisms, as is the example of mesoscale convective tornadoes and storms, which are drivers of severe winds, although these winds have intensities greater than the 99th percentile [51]. The IPCC report "Managing the Risks of Extreme Events and Disasters to Advance Climate Change Adaptation" [24] states that several recent studies reported changes in wind speed in different regions worldwide. However, there is a lack of confidence in projections for small-scale phenomena, such as tornadoes, as competing physical processes can affect future trends and because climate models (global) do not simulate such processes. Thus, there is an urgent need to reduce the uncertainty of climatic projections, namely those related to the wind. Additionally, there is usually little confidence in projections related to extreme winds, owing to the few studies on this.

The present study aims at making a comparison between the historical climate period (1986-2005) and the future climate period (2080-2099) on a regional scale (26 cities on Iberia), while taking into account some variables derived from the wind vector: intensity (wind speed), direction (wind bearing), wind shear, and helicity. For the projection of the future climate, the RCP8.5 (Representative Concentration Pathway 8.5) anthropogenic radiative forcing scenario is used in the regional climate model simulations. Changes of mean wind speed and prevailing wind direction will be identified near the surface. Furthermore, it is possible to study the changes that will occur at upper tropospheric levels, such as changes in the position and intensity of the jet stream. The wind shear and the storm-relative environmental helicity (SREH) will provide a better understanding of the vertical wind changes, allowing for an estimation of the frequency of storms caused by intense vertical movements.

\section{Data and Methods}

The WRF (Weather Research and Forecasting) model was used in the present study. The simulations were carried out with three nested domains and with a two-way coupling procedure [52]. The domains (Figure 1a) have a horizontal resolution of $81 \mathrm{~km}$ (outer/parent domain, D1), $27 \mathrm{~km}$ (intermediate domain, D2) and $9 \mathrm{~km}$ (inner domain, D3). The initial and boundary conditions for these experiments were retrieved from simulations previously generated by the MPI-ESM-LR global climate model (Max Planck Institute Earth System Model-Low Resolution) and the ERA-Interim reanalysis [53,54]. The data were extracted for domain D3 (horizontal resolution of $9 \mathrm{~km}$ ), from which the nearest-point approach was applied to obtain data for the 26 cities in the Iberian Peninsula (Table 1). This network is sufficiently dense for a general assessment of the potential changes in the tropospheric wind characteristics over the Iberian Peninsula. In fact, changes in the mid-latitude wind characteristics above the boundary layer (free atmosphere) tend to be largely dominated by planetary and synoptic-scale anomaly patterns. 


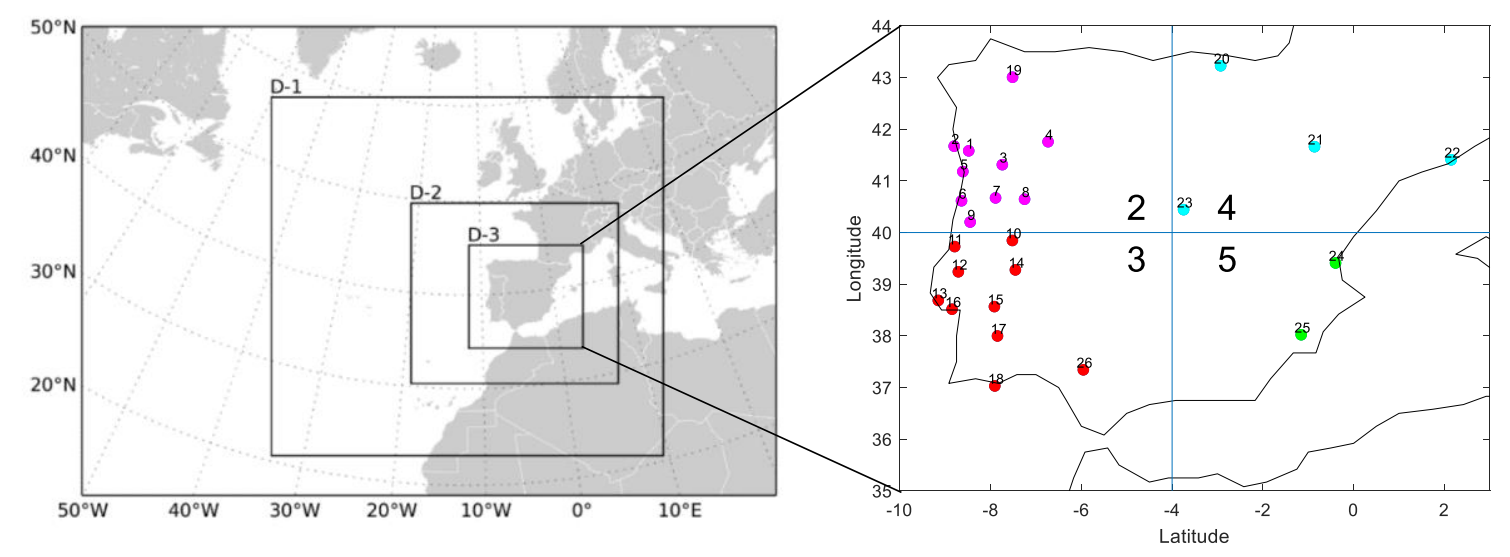

(a)

(b)

Figure 1. (a) The domain used in the Weather Research and Forecasting (WRF) regional implementation, with 81,27 , and $9 \mathrm{~km}$ of horizontal resolution [52] and (b) division of the studied area per regions in the Iberian Peninsula.

Table 1. List of 26 cities considered in this study, along with their corresponding latitude and longitude (negative values of longitude refer to western hemisphere).

\begin{tabular}{|c|c|c|c|}
\hline \multicolumn{4}{|c|}{ Portugal } \\
\hline \multicolumn{2}{|c|}{ Cities } & \multirow{2}{*}{$\begin{array}{c}\text { Latitude }\left({ }^{\circ}\right) \\
41.54\end{array}$} & \multirow{2}{*}{$\begin{array}{c}\text { Longitude }\left({ }^{\circ}\right) \\
-8.43\end{array}$} \\
\hline 1 & Braga & & \\
\hline 2 & Viana do Castelo & 41.67 & -8.75 \\
\hline 3 & Vila Real & 41.31 & -7.74 \\
\hline 4 & Bragança & 41.80 & -6.78 \\
\hline 5 & Porto & 41.17 & -8.58 \\
\hline 6 & Aveiro & 10.75 & -8.67 \\
\hline 7 & Viseu & 40.67 & -7.89 \\
\hline 8 & Guarda & 40.65 & -7.25 \\
\hline 9 & Coimbra & 40.16 & -8.40 \\
\hline 10 & Castelo Branco & 39.80 & -7.47 \\
\hline 11 & Leiria & 39.37 & -8.79 \\
\hline 12 & Santarém & 39.24 & -8.71 \\
\hline 13 & Lisboa & 38.50 & -9.08 \\
\hline 14 & Portalegre & 39.28 & -7.45 \\
\hline 15 & Évora & 38.57 & -7.92 \\
\hline 16 & Setúbal & 38.56 & -8.90 \\
\hline 17 & Beja & 38.00 & -7.85 \\
\hline 18 & Faro & 37.08 & -7.83 \\
\hline \multicolumn{4}{|c|}{ Spain } \\
\hline 19 & Lugo & 43.01 & -7.52 \\
\hline 20 & Bilbao & 43.23 & -2.93 \\
\hline 21 & Zaragoza & 41.63 & -0.92 \\
\hline 22 & Barcelona & 41.33 & 2.08 \\
\hline 23 & Madrid & 40.40 & -3.70 \\
\hline 24 & Valencia & 39.50 & -0.33 \\
\hline 25 & Murcia & 37.98 & -1.11 \\
\hline 26 & Sevilla & 37.39 & -6.00 \\
\hline
\end{tabular}

In this study, the recent historical climate (1986-2005) was obtained from both observation-based data, from ERA-Interim reanalysis, and simulated data, which were generated by the MPI-ESM-LR/WRF model chain. For the future climate (2080-2099), the MPI-ESM-LR/WRF model chain was forced by RCP8.5 [55]. The selected 20-year periods are the same as those considered in the 5th IPCC Assessment Report, AR5 [56]. 
The ERA-Interim reanalysis data were used to validate the simulations that were generated by the MPI-ESM-LR over the historical climate. The model was previously successfully validated by Marta-Almeida et al. [52] by comparison with observations. The ERA-Interim forced simulations were still used for bias correction of the simulated data. In this study, a new bias correction approach was applied using a quantile-quantile calibration method, based on a non-parametric function method that corrects the mean, variability, and shape in the cumulative distribution function (CDF) of the simulated climatic variables. This method consists of correcting the simulated distribution individually relative to observations/reanalyses for each quantile between the observed and simulated data for the past period and apply these to the future projections [57]. This method assumes that the distribution function of the variable may change in the future. The advantage of this approach is the correction of the complete distribution, including the tails, which, in this case, comprises the correction of extreme values of the variables considered. The same method has been applied by other authors $[58,59]$.

The zonal and meridional wind component intensities were extracted for the first 19 levels (isobaric pressure surfaces) from the surface (Table A1). From these, the bulk wind shear (0-6 km), S 0-6, and the storm-relative helicity $(0-3 \mathrm{~km})$, SRH 0-3, were calculated by (1) and (2), respectively:

$$
S 0-6=|\vec{V}(6 k m)-\vec{V}(0)|
$$

where $\vec{V}$ represents the horizontal wind.

$$
\text { SRH 0-3 }=\int_{z=0 k m}^{z=3 k m}(\vec{V}-\vec{C}) \cdot \vec{w}_{h} d z
$$

where $\vec{C}$ is the storm velocity (estimated here as the mean wind velocity between 0 and $3 \mathrm{~km}$ ) and $\vec{w}_{h}$ is the horizontal (zonal and meridional) component of relative vorticity. The dot product means that only the horizontal streamwise vorticity component is relevant for helicity.

The SRH 0-3 is representative of streamwise vorticity within the storm inflow layer and it is used as a tornado forecast tool [60], but is also relevant for non-tornadic supercells [28,31,61]. These studies evaluate S 0-6 and SRH 0-3 for various types of non-supercells, supercells, and tornados in the United States of America. Tables 2 and 3 show these values that were obtained from [28,31], respectively, whereas Table 4 shows typical values of SRH 0-3 for tornados from category F0 to F4 (Fujita scale) reported by [62]. These values are intended to be considered when analyzing the results presented in our study.

Firstly, the average values of the five variables (wind at three level, wind shear, and helicity) were calculated, as well as the corresponding differences between the two time periods (future and historical). A statistical significance test analysis was also carried out ( $t$-student) at a significance level of $5 \%$. Secondly, the complete distribution of the variables is assessed for change between both climates. The objective here is to identify changes across the distribution, particularly in their extreme tails. This is performed by calculating the quantiles for both climates and their difference. This helps to identify differences other than in the mean.

Table 2. Reference values for S 0-6 and storm-relative helicity (SRH) 0-3 from [31]. Values in each cell represent median/75th percentile/90th percentile. F represents the tornado category according to the Fujita scale.

\begin{tabular}{cccc}
\hline Variable & Non-Supercell Storms & $\begin{array}{c}\text { Supercell Storms } \\
\text { Tornados F0 and F1 }\end{array}$ & $\begin{array}{c}\text { Tornadic Supercells } \\
\text { Tornados F2-F5 }\end{array}$ \\
\hline S 0-6 $\left(\mathrm{m} \mathrm{s}^{-1}\right)$ & $10.8 / 15.7 / 22.0$ & $19.1 / 22.1 / 25.8$ & $18.4 / 21.8 / 29.0$ \\
SRH 0-3 $\left(\mathrm{m} \mathrm{s}^{2}\right)$ & $55 / 100 / 168$ & $124 / 208 / 304$ & $180 / 279 / 411$ \\
\hline
\end{tabular}


Table 3. Reference values for SRH 0-3 from [28] for different types of storms. Values in each cell represent median/75th percentile/90th percentile. F represents the tornado category according to the Fujita scale.

\begin{tabular}{ccccc}
\hline Variable & $\begin{array}{c}\text { Non-Supercell } \\
\text { Storms }\end{array}$ & $\begin{array}{c}\text { Non-Tornadic } \\
\text { Supercells }\end{array}$ & $\begin{array}{c}\text { Weakly Tornadic } \\
\text { Storms F0-F1 }\end{array}$ & $\begin{array}{c}\text { Significant Tornadic } \\
\text { Supercells F2-F5 }\end{array}$ \\
\hline S 0-6 $\left(\mathrm{m} \mathrm{s}^{-1}\right)$ & $7.8 / 11.0 / 14.3$ & $22.3 / 27.3 / 31.1$ & $22.8 / 26.8 / 31.1$ & $24.5 / 29.2 / 31.8$ \\
SRH 0-3 $\left(\mathrm{m}^{2} \mathrm{~s}^{-2}\right)$ & - & $146 / 233 / 362$ & $184 / 280 / 367.5$ & $223 / 317 / 396$ \\
\hline
\end{tabular}

Table 4. Reference values for SRH 0-3 from [62] for each tornado category according to the Fujita scale.

\begin{tabular}{cccccc}
\hline Variable & F0 & F1 & F2 & F3 & F4 \\
\hline SRH $0-3\left(\mathrm{~m}^{2} \mathrm{~s}^{-2}\right)$ & 66 & 140 & 196 & 226 & 249 \\
\hline
\end{tabular}

Extreme events were identified and categorized into nine classes/types as a function of their duration and intensity, according to the following method: the duration of an event is the number of hours of the event. Intensity of an event is calculated as the average difference between the variable value minus a threshold of that variable (i.e., 90th percentile computed over the full series for the historical period) for the event. Next, for each city, the 25th and 90th percentiles of intensity were determined and, together with the duration threshold of $6 \mathrm{~h}$ and $36 \mathrm{~h}$, were used as the criterion to allocate an event to a category. Hence, all of the events for each variable are allocated to an event type from 1 to 9 . Figure 2 shows the matrix of the types of events considered. Furthermore, the percentiles and the corresponding event classification were first calculated for each city, separately. For further analysis, the event classification was also performed by season (winter: December to February, spring: March to May, summer: June to August, autumn: September to November) and aggregated for the whole Iberia (1) and in four sub-regions $(2,3,4$, and 5, Figure $1 \mathrm{~b})$, which correspond to the quadrants of the study area. Region 2 represents the north-western quadrant, region 3 is southwest, region 4 northeast, and region 5 southeast. This division is somewhat subjective although it represents crude regions of common synoptic behavior. However, we also show results for individual stations and do not fully rely on the regional division. Additionally, most of the analysis is performed and shown for individual stations. Only in 3.4 (extreme events by type) the regional division is used in order to minimize the amount of results to be shown.

Finally, the return periods of the extreme events were calculated for both the number of events per year, using the Weibull distribution moments method [63], and the duration of events, while using the Gumbel distribution method [64]. Among a large pool of theoretical distribution functions, the latter two were eventually chosen, since they provide the best adjustments to the empirical distributions, according to the Kolmogorov-Smirnov test (not shown). The return periods are estimated for each climate when considering that it is a sample of the same population (stationary climate). 


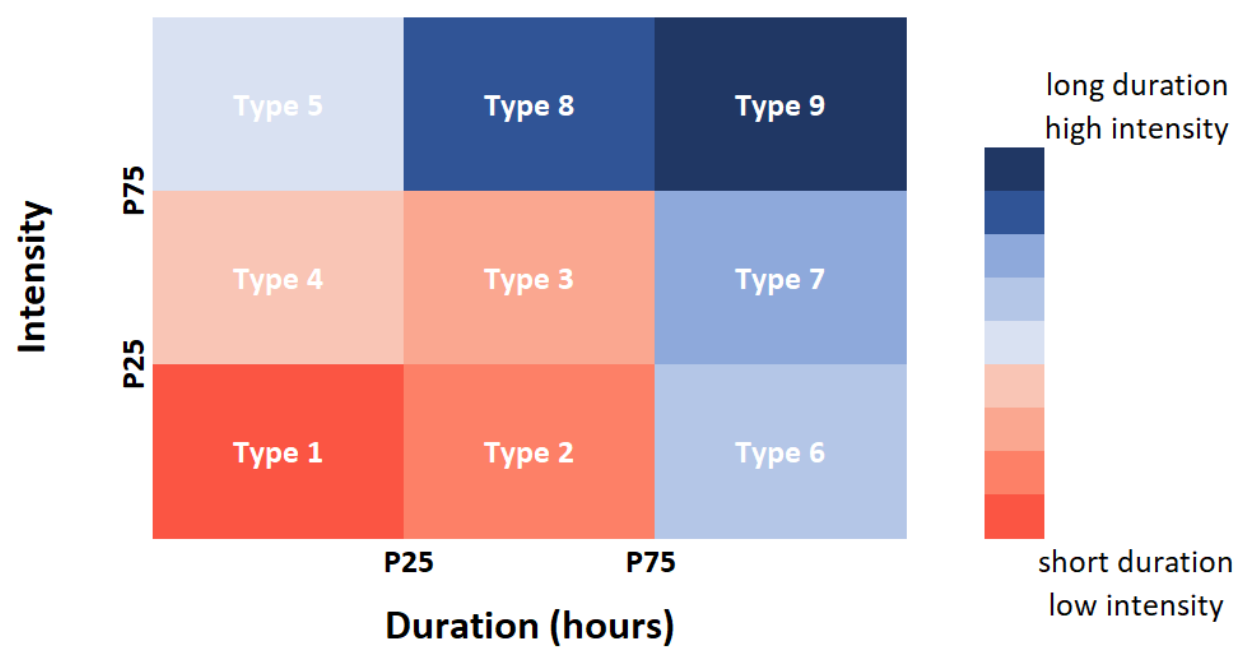

Figure 2. Matrix of the possible extreme events, considering intensity and duration.

\section{Results}

\subsection{Average Changes}

\subsubsection{Surface Wind}

Table 5 shows the intensity of the monthly and annual mean wind at the surface, for each city, for the historical period and the difference between the future and historical periods. In general, the intensity of the average wind decreases for all months, except June, July, and September, when it increases. In regional terms, it can be concluded that, throughout Spain, the average wind speed will decrease, except for Lugo, while in Portugal there is a division between cities to the north and south of Santarém. The cities northwards of Santarém (inclusive) and Faro have lower wind speed at the surface for the future, while cities to the south show average increases in this variable.

Wind roses for each city, for both the historical and future climate, were produced. Wind roses were drawn for 16 directions. Figure 3 represents the wind rose for Lisbon for historical (a) and future (b) periods. This city is representative of the results that were found for the majority of the cities. It is shown that the predominant wind directions will be maintained and, in most cases, will be more frequent.

(a)

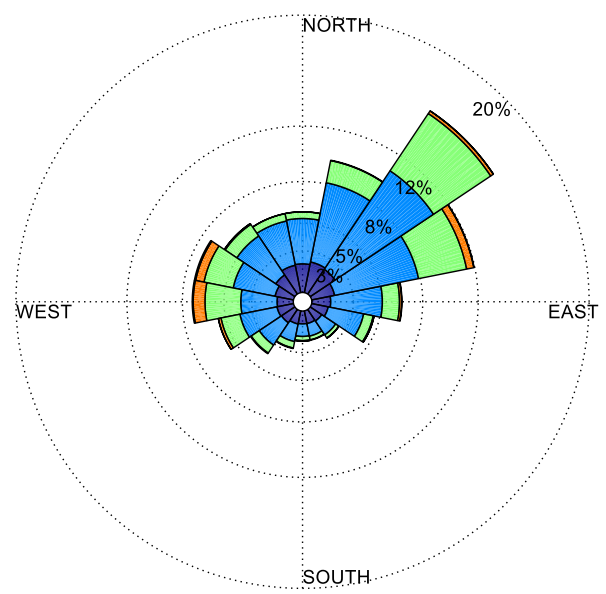

(b)

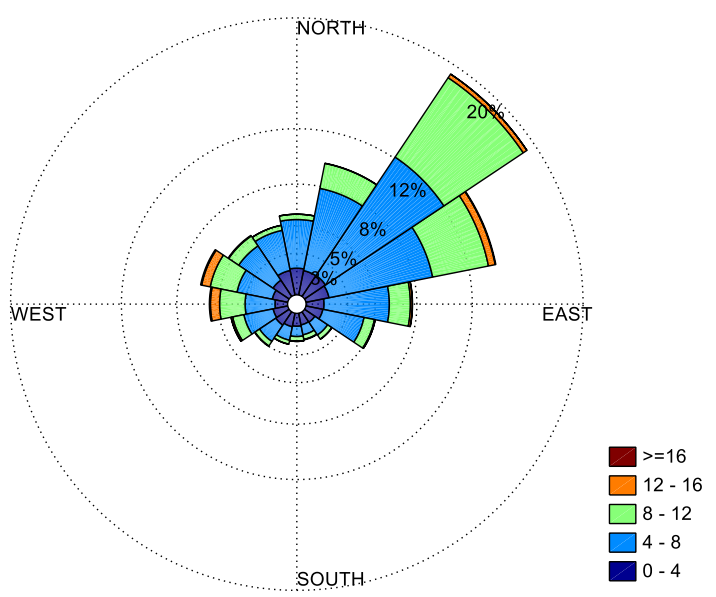

Figure 3. Wind roses for wind at the surface in Lisboa for (a) historical and (b) future climates. Each colour represents an interval of wind speed. Sixteen directional divisions. The circles mark the number of occurrence (in percentage) for each wind direction (16 direction bars were considered). 
Table 5. Monthly and annual averaged wind speed near the surface for each city and the difference between the future and the historical climates. Significant differences ( $t$-student $5 \%$ ) are shown in red (positive) and blue (negative).

\begin{tabular}{|c|c|c|c|c|c|c|c|c|c|c|c|c|c|c|}
\hline \multirow{2}{*}{\multicolumn{2}{|c|}{ CITIES }} & \multicolumn{13}{|c|}{ Months } \\
\hline & & January & February & March & April & May & June & July & August & September & October & November & December & Year \\
\hline \multirow{2}{*}{ Braga } & MPI-hist & 7.05 & 6.63 & 5.66 & 5.20 & 4.28 & 3.69 & 3.74 & 3.77 & 4.07 & 4.78 & 5.76 & 6.53 & 5.09 \\
\hline & Differ. & -0.49 & -0.02 & -0.25 & -0.40 & -0.20 & -0.01 & 0.10 & -0.08 & -0.34 & -0.55 & 0.03 & -0.42 & -0.22 \\
\hline \multirow{2}{*}{$\begin{array}{l}\text { Viana do } \\
\text { Castelo }\end{array}$} & MPI-hist & 8.57 & 8.33 & 7.40 & 6.86 & 6.29 & 5.91 & 6.46 & 6.37 & 6.21 & 6.41 & 7.23 & 8.12 & 7.01 \\
\hline & Differ. & -0.62 & -0.08 & -0.48 & -0.17 & -0.10 & 0.46 & 0.26 & 0.26 & -0.05 & -0.62 & 0.01 & -0.44 & -0.13 \\
\hline \multirow{2}{*}{ Vila Real } & MPI-hist & 6.89 & 6.80 & 6.00 & 5.73 & 5.02 & 4.29 & 4.64 & 4.68 & 4.88 & 5.04 & 5.88 & 6.53 & 5.53 \\
\hline & Differ. & -0.44 & -0.22 & -0.45 & -0.39 & -0.26 & 0.23 & 0.03 & -0.10 & -0.17 & -0.13 & -0.10 & -0.63 & -0.22 \\
\hline \multirow{2}{*}{ Bragança } & MPI-hist & 5.96 & 6.01 & 5.67 & 5.55 & 5.43 & 4.80 & 5.01 & 5.15 & 5.10 & 5.07 & 5.51 & 5.65 & 5.41 \\
\hline & Differ. & -0.35 & -0.34 & -0.49 & -0.14 & -0.23 & 0.14 & 0.02 & -0.36 & -0.16 & -0.03 & -0.30 & -0.72 & -0.25 \\
\hline \multirow{2}{*}{ Porto } & MPI-hist & 7.96 & 7.44 & 6.05 & 5.49 & 4.57 & 3.89 & 4.12 & 4.04 & 4.27 & 4.82 & 6.03 & 7.34 & 5.50 \\
\hline & Differ. & -0.81 & -0.21 & -0.41 & -0.49 & -0.29 & 0.07 & -0.07 & -0.18 & -0.35 & -0.69 & -0.10 & -0.70 & -0.36 \\
\hline \multirow{2}{*}{ Aveiro } & MPI-hist & 9.34 & 8.91 & 7.59 & 7.09 & 6.08 & 5.25 & 5.53 & 5.53 & 5.66 & 6.24 & 7.44 & 8.79 & 6.94 \\
\hline & Differ. & -0.68 & -0.07 & -0.47 & -0.52 & -0.29 & 0.14 & -0.17 & -0.45 & -0.33 & -0.75 & -0.07 & -0.60 & -0.36 \\
\hline \multirow{2}{*}{ Viseu } & MPI-hist & 4.33 & 4.29 & 4.15 & 4.10 & 3.89 & 3.68 & 3.81 & 3.78 & 3.71 & 3.64 & 3.92 & 3.99 & 3.94 \\
\hline & Differ. & -0.25 & -0.15 & -0.33 & -0.16 & -0.03 & 0.17 & -0.01 & -0.17 & -0.14 & -0.15 & -0.19 & -0.26 & -0.14 \\
\hline \multirow{2}{*}{ Guarda } & MPI-hist & 6.26 & 6.02 & 5.68 & 5.03 & 4.20 & 3.78 & 3.73 & 3.79 & 4.34 & 5.34 & 6.10 & 6.00 & 5.02 \\
\hline & Differ. & 0.27 & 0.02 & -0.46 & -0.33 & -0.09 & -0.01 & 0.16 & -0.20 & -0.35 & -0.74 & -0.10 & 0.36 & -0.12 \\
\hline \multirow{2}{*}{ Coimbra } & MPI-hist & 6.58 & 6.15 & 5.48 & 5.05 & 4.34 & 3.87 & 3.98 & 4.01 & 4.28 & 5.02 & 6.05 & 6.50 & 5.10 \\
\hline & Differ. & -0.10 & -0.01 & -0.38 & -0.29 & 0.06 & 0.04 & -0.11 & -0.20 & -0.30 & -0.55 & -0.11 & -0.27 & -0.19 \\
\hline Castelo & MPI-hist & 6.33 & 6.07 & 5.57 & 5.32 & 4.74 & 4.72 & 5.03 & 5.11 & 5.07 & 5.23 & 5.78 & 6.21 & 5.43 \\
\hline Branco & Differ. & -0.32 & -0.32 & -0.50 & -0.32 & 0.08 & 0.29 & 0.29 & -0.10 & -0.02 & 0.07 & -0.16 & -0.70 & -0.14 \\
\hline \multirow[b]{2}{*}{ Leiria } & MPI-hist & 7.28 & 6.76 & 6.08 & 5.90 & 5.30 & 4.62 & 5.02 & 4.97 & 5.00 & 5.42 & 6.51 & 7.17 & 5.83 \\
\hline & Differ. & -0.60 & -0.09 & -0.41 & -0.30 & -0.09 & 0.23 & -0.23 & -0.16 & -0.12 & -0.28 & -0.15 & -0.77 & -0.25 \\
\hline \multirow{2}{*}{ Santarém } & MPI-hist & 6.22 & 5.88 & 5.45 & 5.27 & 4.61 & 4.46 & 4.76 & 5.01 & 4.96 & 5.18 & 5.72 & 5.99 & 5.29 \\
\hline & Differ. & -0.23 & -0.21 & -0.39 & -0.34 & 0.12 & 0.20 & 0.01 & -0.26 & -0.01 & -0.03 & -0.06 & -0.58 & -0.15 \\
\hline \multirow{2}{*}{ Lisboa } & MPI-hist & 6.43 & 6.35 & 6.05 & 6.15 & 5.92 & 6.20 & 6.87 & 6.87 & 6.14 & 5.77 & 5.99 & 6.17 & 6.24 \\
\hline & Differ. & -0.11 & -0.33 & -0.25 & -0.14 & 0.28 & 0.76 & 0.39 & 0.20 & 0.46 & 0.28 & -0.06 & -0.49 & 0.08 \\
\hline \multirow{2}{*}{ Portalegre } & MPI-hist & 6.86 & 6.74 & 6.25 & 6.18 & 5.73 & 6.03 & 6.45 & 6.47 & 5.97 & 5.73 & 6.17 & 6.48 & 6.25 \\
\hline & Differ. & -0.29 & -0.38 & -0.30 & -0.17 & 0.29 & 0.50 & 0.54 & 0.05 & 0.32 & 0.31 & -0.11 & -0.56 & 0.02 \\
\hline
\end{tabular}


Table 5. Cont

\begin{tabular}{|c|c|c|c|c|c|c|c|c|c|c|c|c|c|c|}
\hline \multirow{2}{*}{\multicolumn{2}{|c|}{ CITIES }} & \multicolumn{13}{|c|}{ Months } \\
\hline & & January & February & March & April & May & June & July & August & September & October & November & December & Year \\
\hline \multirow{2}{*}{ Évora } & MPI-hist & 7.15 & 7.08 & 6.55 & 6.81 & 6.43 & 6.93 & 7.49 & 7.40 & 6.57 & 6.19 & 6.48 & 6.80 & 6.82 \\
\hline & Differ. & -0.17 & -0.19 & 0.01 & -0.17 & 0.39 & 0.71 & 0.50 & 0.18 & 0.64 & 0.29 & 0.07 & -0.43 & 0.15 \\
\hline \multirow{2}{*}{ Setúbal } & MPI-hist & 6.76 & 6.73 & 6.38 & 6.56 & 6.32 & 6.79 & 7.40 & 7.34 & 6.49 & 6.04 & 6.21 & 6.44 & 6.62 \\
\hline & Differ. & -0.13 & -0.26 & -0.09 & -0.11 & 0.37 & 0.81 & 0.49 & 0.25 & 0.59 & 0.29 & 0.02 & -0.39 & 0.15 \\
\hline \multirow{2}{*}{ Beja } & MPI-hist & 7.23 & 7.11 & 6.50 & 6.95 & 6.40 & 6.76 & 7.00 & 6.97 & 6.16 & 5.99 & 6.37 & 6.95 & 6.70 \\
\hline & Differ. & -0.38 & -0.28 & 0.00 & -0.40 & 0.23 & 0.55 & 0.53 & 0.13 & 0.70 & 0.07 & 0.05 & -0.72 & 0.04 \\
\hline \multirow{2}{*}{ Faro } & MPI-hist & 6.45 & 6.28 & 5.60 & 5.69 & 4.67 & 4.18 & 3.80 & 4.03 & 4.51 & 5.48 & 6.22 & 6.29 & 5.26 \\
\hline & Differ. & -0.11 & -0.42 & 0.09 & -0.60 & -0.22 & -0.24 & -0.43 & -0.78 & -0.32 & -0.88 & -0.89 & -0.71 & -0.46 \\
\hline \multirow{2}{*}{ Lugo } & MPI-hist & 6.60 & 6.46 & 6.07 & 5.64 & 5.18 & 5.25 & 4.96 & 5.04 & 5.36 & 5.65 & 6.30 & 6.47 & 5.74 \\
\hline & Differ. & 0.13 & 0.27 & 0.07 & 0.00 & 0.09 & -0.01 & 0.77 & 0.61 & -0.02 & -0.21 & -0.10 & 0.18 & 0.15 \\
\hline \multirow[b]{2}{*}{ Bilbao } & MPI-hist & 6.74 & 6.48 & 5.58 & 4.95 & 4.24 & 3.58 & 3.35 & 3.51 & 3.87 & 4.86 & 5.86 & 6.50 & 4.95 \\
\hline & Differ. & -0.25 & -0.31 & -0.33 & -0.49 & -0.34 & -0.26 & -0.19 & -0.40 & -0.19 & -0.57 & -0.33 & -0.07 & -0.31 \\
\hline \multirow{2}{*}{ Zaragoza } & MPI-hist & 7.12 & 6.95 & 6.14 & 6.41 & 5.27 & 4.80 & 4.57 & 4.66 & 4.90 & 5.56 & 6.36 & 7.15 & 5.82 \\
\hline & Differ. & -0.53 & -0.30 & -0.20 & -0.67 & -0.08 & -0.28 & -0.13 & -0.31 & -0.16 & -0.33 & -0.64 & -1.13 & -0.40 \\
\hline \multirow[b]{2}{*}{ Barcelona } & MPI-hist & 5.40 & 5.18 & 4.93 & 4.92 & 4.39 & 4.14 & 3.96 & 4.17 & 4.48 & 4.92 & 5.29 & 5.38 & 4.76 \\
\hline & Differ. & -0.17 & -0.28 & -0.12 & -0.28 & 0.03 & -0.21 & -0.05 & -0.15 & -0.16 & -0.15 & -0.37 & -0.59 & -0.21 \\
\hline \multirow{2}{*}{ Madrid } & MPI-hist & 5.97 & 5.75 & 5.38 & 5.09 & 4.67 & 4.44 & 4.52 & 4.52 & 4.72 & 4.69 & 5.38 & 5.61 & 5.06 \\
\hline & Differ. & -0.12 & -0.12 & -0.17 & -0.10 & 0.09 & -0.06 & 0.14 & -0.30 & -0.28 & 0.19 & -0.27 & -0.40 & -0.12 \\
\hline \multirow{2}{*}{ Valencia } & MPI-hist & 5.67 & 5.38 & 5.27 & 5.14 & 4.47 & 4.39 & 4.11 & 4.32 & 4.66 & 5.26 & 5.91 & 5.90 & 5.04 \\
\hline & Differ. & 0.00 & -0.21 & -0.11 & -0.31 & 0.12 & -0.08 & 0.09 & -0.27 & -0.04 & -0.09 & -0.39 & -0.34 & -0.14 \\
\hline \multirow{2}{*}{ Murcia } & MPI-hist & 4.38 & 4.34 & 4.06 & 4.42 & 3.84 & 3.33 & 3.15 & 3.22 & 3.24 & 3.52 & 3.89 & 4.06 & 3.78 \\
\hline & Differ. & -0.17 & -0.19 & -0.08 & -0.45 & -0.18 & -0.12 & 0.04 & -0.14 & -0.08 & -0.26 & -0.25 & -0.39 & -0.19 \\
\hline \multirow{2}{*}{ Sevilla } & MPI-hist & 5.81 & 5.60 & 5.02 & 5.16 & 3.98 & 3.59 & 3.08 & 3.27 & 3.63 & 4.80 & 5.58 & 5.83 & 4.61 \\
\hline & Differ. & -0.06 & -0.25 & 0.21 & -0.66 & -0.19 & -0.24 & -0.18 & -0.54 & -0.20 & -0.81 & -0.64 & -0.72 & -0.36 \\
\hline
\end{tabular}




\subsubsection{Wind at $850 \mathrm{hPa}$}

Table 6 shows the average monthly and annual wind speed at $850 \mathrm{hPa}$ for each city, for the historical period and the difference between the future and historical periods. As for the wind at the surface, statistically significant negative differences are observed for all cities, particularly for summer (56\% differences), October ( $96 \%$ of the differences), and annual conditions ( $77 \%$ of the differences). Overall, the wind speed is projected to decrease in the future at $850 \mathrm{hPa}$, without much distinction between cities. Differences between near-surface and $850 \mathrm{hPa}$ wind speed represent, to a large extent, the planetary boundary layer (PBL) effects, whereas the $850 \mathrm{hPa}$ is typically in the transition from the PBL to the free atmosphere.

\subsubsection{Wind at $300 \mathrm{hPa}$}

Table 7 presents the wind speed at $300 \mathrm{hPa}$. It depicts an evident distinction between the period from June to October from the remaining months. Within the former period, the differences are negative and statistically significant, while the differences are positive, but rarely statistically significant, for the remaining months. No clear spatial pattern is found among the cities.

\subsubsection{Wind Shear $(0-6 \mathrm{~km})$}

Table 8 shows the wind shear intensity for the 0 to $6 \mathrm{~km}$ layer for each city, for the historical period, and the difference between the future and historical periods. The intensity of the wind shear shows negative differences between July and September, while the differences are mostly positive for the remaining months.

\subsubsection{Helicity $(0-3 \mathrm{~km})$}

Table 9 shows the helicity between 0 and $3 \mathrm{~km}$ for each city, for the historical period and the difference between the future and historical period. More positive and statistically significant differences are observed for the months between June and September. For annual conditions, most of the statistically significant data reveal a positive difference (strengthening of helicity).

The changes in helicity themselves are equal to or higher than the respective average values recorded in the historical period ( $>100 \%)$. For example, for Vila Real, Guarda, Leiria, and Beja, in August, the differences exceed twice the average value of the historical period. It can be concluded that, in general, the helicity will tend to increase, in some regions (for example: Bragança, Guarda, and Beja) by more than $200 \%$ as compared to the historical period. However, for some cities, there will be a decrease in helicity, as in the case of Braga, Bragança, Guarda, Coimbra, and Bilbao. Although Guarda has mostly negative (not significant) differences, significant positive differences of over $200 \%$ are observed in July and August.

The main conclusions presented in Tables 5-9 are summarized in Figures 4-9. For periods and variables that are not shown here, the changes are not temporally or spatially consistent and are, therefore, not shown. Figure 4 shows that wind speed near the surface is expected to decrease most of the time during the year, except for Lisboa and Portalegre (50\%), Setúbal (42\%), and Évora, Beja, and Lugo (33\%).

For wind speed at $850 \mathrm{hPa}$ (Figure 5), the whole region is expected to register a reduction in the future for the May-October period. Upper tropospheric winds ( $300 \mathrm{hPa}$ ) (Figure 6) will reduce for the whole region during all time (i.e., 100\%) for the May-September period. Wind shear 0-6 km is expected to increase for the June-November period (Figure 7) and decrease (Figure 8) between July and October. Storm-relative helicity 0-3 km (Figure 9) will increase during summer (June-September) in most of the region during most of the time. 
Table 6. Monthly and annual averaged wind speed at $850 \mathrm{hPa}$ for each city and the difference between the future and the historical climates. Significant differences ( $t$-student $5 \%$ ) are shown in red (positive) and blue (negative).

\begin{tabular}{|c|c|c|c|c|c|c|c|c|c|c|c|c|c|c|}
\hline \multirow{2}{*}{\multicolumn{2}{|c|}{ CITIES }} & \multicolumn{13}{|c|}{ Months } \\
\hline & & January & February & March & April & May & June & July & August & September & October & November & December & Year \\
\hline \multirow{2}{*}{ Braga } & MPI-hist & 12.69 & 12.01 & 10.70 & 9.38 & 7.63 & 6.61 & 6.41 & 6.44 & 7.33 & 9.29 & 10.83 & 12.09 & 9.27 \\
\hline & Differ. & 0.06 & 0.45 & -0.04 & -0.12 & 0.00 & -0.42 & -0.53 & -0.96 & -0.88 & -1.60 & 0.12 & 0.14 & -0.32 \\
\hline \multirow{2}{*}{$\begin{array}{l}\text { Viana do } \\
\text { Castelo }\end{array}$} & MPI-hist & 12.99 & 12.27 & 10.93 & 9.44 & 7.64 & 6.50 & 6.03 & 6.19 & 7.31 & 9.43 & 11.13 & 12.49 & 9.35 \\
\hline & Differ. & 0.01 & 0.43 & -0.17 & -0.13 & -0.10 & -0.53 & -0.47 & -1.04 & -1.01 & -1.65 & 0.14 & 0.09 & -0.38 \\
\hline \multirow{2}{*}{ Vila Real } & MPI-hist & 12.59 & 11.95 & 10.70 & 9.59 & 7.93 & 7.09 & 7.29 & 7.12 & 7.63 & 9.33 & 10.66 & 11.89 & 9.47 \\
\hline & Differ. & 0.08 & 0.48 & 0.18 & 0.08 & 0.18 & -0.23 & -0.48 & -0.77 & -0.61 & -1.39 & 0.16 & 0.17 & -0.18 \\
\hline \multirow{2}{*}{ Bragança } & MPI-hist & 11.89 & 11.16 & 10.06 & 8.83 & 7.21 & 6.13 & 5.78 & 5.97 & 6.86 & 8.57 & 9.99 & 11.08 & 8.62 \\
\hline & Differ. & -0.45 & 0.11 & -0.21 & -0.01 & -0.06 & -0.67 & -0.48 & -1.10 & -0.75 & -1.16 & -0.08 & -0.18 & -0.42 \\
\hline \multirow[b]{2}{*}{ Porto } & MPI-hist & 12.60 & 12.00 & 10.77 & 9.46 & 7.73 & 6.61 & 6.62 & 6.54 & 7.39 & 9.20 & 10.71 & 11.93 & 9.29 \\
\hline & Differ. & 0.21 & 0.45 & -0.02 & -0.19 & 0.04 & -0.26 & -0.55 & -0.89 & -0.86 & -1.51 & 0.14 & 0.24 & -0.27 \\
\hline \multirow{2}{*}{ Aveiro } & MPI-hist & 12.95 & 12.32 & 10.98 & 9.71 & 7.94 & 6.80 & 7.03 & 6.77 & 7.56 & 9.28 & 10.90 & 12.11 & 9.52 \\
\hline & Differ. & 0.26 & 0.42 & 0.10 & -0.26 & 0.11 & -0.07 & -0.54 & -0.64 & -0.85 & -1.43 & 0.14 & 0.18 & -0.22 \\
\hline \multirow{2}{*}{ Viseu } & MPI-hist & 10.51 & 9.99 & 8.99 & 7.97 & 6.70 & 5.80 & 5.50 & 5.59 & 6.25 & 7.76 & 9.00 & 9.70 & 7.80 \\
\hline & Differ. & -0.12 & -0.26 & -0.23 & -0.10 & -0.09 & -0.41 & -0.32 & -0.78 & -0.50 & -1.16 & -0.25 & -0.14 & -0.36 \\
\hline \multirow{2}{*}{ Guarda } & MPI-hist & 10.25 & 9.88 & 9.10 & 8.26 & 7.11 & 5.95 & 5.69 & 5.77 & 6.35 & 7.86 & 9.07 & 9.50 & 7.89 \\
\hline & Differ. & -0.10 & -0.32 & -0.27 & 0.11 & -0.19 & -0.49 & -0.31 & -0.91 & -0.42 & -1.14 & -0.44 & -0.30 & -0.40 \\
\hline \multirow{2}{*}{ Coimbra } & MPI-hist & 10.10 & 9.75 & 8.82 & 8.08 & 6.87 & 5.79 & 5.41 & 5.48 & 6.17 & 7.53 & 8.75 & 9.39 & 7.67 \\
\hline & Differ. & 0.01 & -0.32 & -0.24 & -0.28 & -0.21 & -0.40 & -0.34 & -0.78 & -0.57 & -1.12 & -0.37 & -0.25 & -0.41 \\
\hline Castelo & MPI-hist & 9.84 & 9.57 & 8.65 & 8.05 & 6.74 & 5.53 & 4.97 & 5.17 & 5.94 & 7.50 & 8.62 & 9.16 & 7.47 \\
\hline Branco & Differ. & 0.06 & -0.17 & -0.12 & -0.36 & -0.36 & -0.60 & -0.28 & -0.76 & -0.50 & -1.21 & -0.33 & -0.12 & -0.40 \\
\hline \multirow[b]{2}{*}{ Leiria } & MPI-hist & 10.17 & 9.85 & 8.96 & 8.33 & 7.08 & 5.88 & 5.44 & 5.53 & 6.19 & 7.65 & 8.85 & 9.49 & 7.77 \\
\hline & Differ. & 0.04 & -0.36 & -0.35 & -0.45 & -0.33 & -0.39 & -0.40 & -0.78 & -0.52 & -1.13 & -0.35 & -0.31 & -0.45 \\
\hline \multirow{2}{*}{ Santarém } & MPI-hist & 9.87 & 9.62 & 8.73 & 8.19 & 6.89 & 5.69 & 5.11 & 5.23 & 6.01 & 7.53 & 8.74 & 9.25 & 7.56 \\
\hline & Differ. & 0.09 & -0.31 & -0.25 & -0.54 & -0.40 & -0.43 & -0.17 & -0.57 & -0.46 & -1.19 & -0.42 & -0.31 & -0.41 \\
\hline \multirow{2}{*}{ Lisboa } & MPI-hist & 10.01 & 9.88 & 8.88 & 8.45 & 7.13 & 5.79 & 5.28 & 5.33 & 6.08 & 7.63 & 8.88 & 9.42 & 7.72 \\
\hline & Differ. & 0.11 & -0.47 & -0.34 & -0.66 & -0.47 & -0.36 & -0.07 & -0.47 & -0.36 & -1.22 & -0.49 & -0.50 & -0.44 \\
\hline \multirow{2}{*}{ Portalegre } & MPI-hist & 10.32 & 10.07 & 8.97 & 8.58 & 7.13 & 5.63 & 5.13 & 5.18 & 5.99 & 7.70 & 8.93 & 9.47 & 7.75 \\
\hline & Differ. & -0.03 & -0.24 & -0.17 & -0.62 & -0.58 & -0.61 & -0.15 & -0.51 & -0.45 & -1.37 & -0.45 & -0.30 & -0.46 \\
\hline
\end{tabular}


Table 6. Cont

\begin{tabular}{|c|c|c|c|c|c|c|c|c|c|c|c|c|c|c|}
\hline \multirow{2}{*}{\multicolumn{2}{|c|}{ CITIES }} & \multicolumn{13}{|c|}{ Months } \\
\hline & & January & February & March & April & May & June & July & August & September & October & November & December & Year \\
\hline \multirow{2}{*}{ Évora } & MPI-hist & 10.53 & 10.38 & 9.02 & 8.81 & 7.17 & 5.43 & 5.02 & 5.09 & 5.88 & 7.60 & 9.04 & 9.59 & 7.78 \\
\hline & Differ. & -0.08 & -0.46 & -0.18 & -0.84 & -0.69 & -0.43 & -0.14 & -0.49 & -0.52 & -1.38 & -0.55 & -0.53 & -0.52 \\
\hline \multirow{2}{*}{ Setúbal } & MPI-hist & 10.19 & 10.11 & 8.95 & 8.61 & 7.20 & 5.68 & 5.17 & 5.25 & 5.99 & 7.61 & 8.97 & 9.47 & 7.75 \\
\hline & Differ. & 0.06 & -0.50 & -0.29 & -0.75 & -0.55 & -0.37 & -0.08 & -0.51 & -0.38 & -1.29 & -0.51 & -0.54 & -0.48 \\
\hline \multirow{2}{*}{ Beja } & MPI-hist & 10.80 & 10.59 & 9.08 & 9.01 & 7.21 & 5.27 & 5.14 & 5.02 & 5.79 & 7.47 & 9.05 & 9.70 & 7.83 \\
\hline & Differ. & -0.20 & -0.53 & -0.15 & -0.95 & -0.74 & -0.32 & -0.32 & -0.44 & -0.53 & -1.34 & -0.56 & -0.74 & -0.57 \\
\hline \multirow{2}{*}{ Faro } & MPI-hist & 9.68 & 9.29 & 7.94 & 8.18 & 6.49 & 5.58 & 4.78 & 5.12 & 5.83 & 7.38 & 8.39 & 9.06 & 7.30 \\
\hline & Differ. & -0.17 & -0.47 & 0.35 & -0.76 & -0.38 & -0.23 & -0.06 & -0.99 & -0.43 & -1.40 & -0.84 & -0.86 & -0.52 \\
\hline \multirow{2}{*}{ Lugo } & MPI-hist & 13.60 & 13.00 & 11.56 & 9.86 & 8.11 & 6.97 & 6.55 & 6.78 & 7.92 & 10.13 & 11.79 & 13.15 & 9.94 \\
\hline & Differ. & -0.10 & 0.46 & -0.03 & 0.20 & 0.00 & -0.61 & -0.74 & -1.39 & -1.04 & -1.74 & 0.13 & 0.11 & -0.40 \\
\hline \multirow{2}{*}{ Bilbao } & MPI-hist & 12.17 & 11.40 & 10.30 & 8.80 & 7.72 & 7.01 & 7.51 & 7.39 & 7.82 & 9.07 & 10.31 & 11.63 & 9.25 \\
\hline & Differ. & -0.34 & 0.62 & 0.20 & 0.83 & 0.52 & 0.12 & -0.26 & -0.93 & -0.57 & -1.04 & 0.00 & 0.03 & -0.08 \\
\hline \multirow{2}{*}{ Zaragoza } & MPI-hist & 12.47 & 11.81 & 10.27 & 9.81 & 7.99 & 6.30 & 5.83 & 6.22 & 6.93 & 8.73 & 10.43 & 12.00 & 9.05 \\
\hline & Differ. & -0.92 & 0.14 & -0.18 & -0.62 & -0.52 & -0.69 & -0.40 & -1.40 & -0.77 & -0.96 & -0.13 & -0.92 & -0.62 \\
\hline \multirow[b]{2}{*}{ Barcelona } & MPI-hist & 10.74 & 10.01 & 8.68 & 7.98 & 6.51 & 5.35 & 4.78 & 5.22 & 6.01 & 7.51 & 8.97 & 10.61 & 7.69 \\
\hline & Differ. & -0.47 & 0.44 & 0.19 & -0.28 & -0.28 & -0.64 & -0.47 & -1.33 & -1.01 & -1.06 & -0.02 & -0.82 & -0.49 \\
\hline \multirow{2}{*}{ Madrid } & MPI-hist & 10.70 & 10.36 & 9.21 & 8.33 & 7.11 & 5.56 & 5.47 & 5.53 & 6.37 & 7.72 & 9.14 & 9.76 & 7.93 \\
\hline & Differ. & -0.32 & -0.12 & -0.20 & 0.12 & -0.27 & -0.40 & -0.37 & -0.73 & -0.83 & -0.94 & -0.54 & -0.30 & -0.41 \\
\hline \multirow{2}{*}{ Valencia } & MPI-hist & 10.74 & 10.09 & 8.49 & 8.17 & 6.20 & 5.25 & 4.57 & 4.72 & 5.57 & 7.13 & 8.60 & 10.20 & 7.46 \\
\hline & Differ. & -0.54 & 0.22 & 0.14 & -0.74 & -0.36 & -0.60 & 0.04 & -0.77 & -0.85 & -1.31 & 0.02 & -0.97 & -0.48 \\
\hline \multirow[b]{2}{*}{ Murcia } & MPI-hist & 10.24 & 9.67 & 8.19 & 7.92 & 6.11 & 5.10 & 4.45 & 4.69 & 5.44 & 7.07 & 8.36 & 9.46 & 7.21 \\
\hline & Differ. & -0.42 & -0.18 & 0.17 & -0.73 & -0.38 & -0.40 & 0.05 & -0.57 & -0.60 & -1.27 & -0.33 & -0.94 & -0.47 \\
\hline \multirow{2}{*}{ Sevilla } & MPI-hist & 9.65 & 9.08 & 7.71 & 7.80 & 6.12 & 5.41 & 4.62 & 4.91 & 5.64 & 7.21 & 8.26 & 8.84 & 7.09 \\
\hline & Differ. & -0.39 & -0.32 & 0.40 & -0.69 & -0.42 & -0.39 & 0.20 & -0.68 & -0.37 & -1.26 & -0.68 & -0.77 & -0.45 \\
\hline
\end{tabular}


Table 7. Monthly and annual averaged wind speed at $300 \mathrm{hPa}$ for each city and the difference between the future and the historical climates. Significant differences ( $t$-student $5 \%$ ) are shown in red (positive) and blue (negative).

\begin{tabular}{|c|c|c|c|c|c|c|c|c|c|c|c|c|c|c|}
\hline \multirow{2}{*}{\multicolumn{2}{|c|}{ CITIES }} & \multicolumn{13}{|c|}{ Months } \\
\hline & & January & February & March & April & May & June & July & August & September & October & November & December & Year \\
\hline \multirow{2}{*}{ Braga } & MPI-hist & 28.21 & 27.46 & 24.36 & 24.07 & 21.47 & 18.23 & 19.51 & 18.68 & 20.19 & 23.37 & 24.76 & 26.45 & 23.04 \\
\hline & Differ. & 2.83 & 3.15 & 2.76 & 1.57 & 2.14 & 0.16 & -3.09 & -3.24 & -2.31 & -2.06 & 3.18 & 1.76 & 0.55 \\
\hline \multirow{2}{*}{$\begin{array}{l}\text { Viana do } \\
\text { Castelo }\end{array}$} & MPI-hist & 28.20 & 27.42 & 24.30 & 24.07 & 21.47 & 18.15 & 19.31 & 18.62 & 20.20 & 23.48 & 24.83 & 26.49 & 23.03 \\
\hline & Differ. & 2.88 & 3.16 & 2.74 & 1.40 & 1.96 & 0.15 & -2.96 & -3.29 & -2.41 & -2.15 & 3.18 & 1.85 & 0.52 \\
\hline \multirow{2}{*}{ Vila Real } & MPI-hist & 28.17 & 27.43 & 24.36 & 24.07 & 21.48 & 18.39 & 19.90 & 18.91 & 20.30 & 23.30 & 24.68 & 26.33 & 23.0 \\
\hline & Differ. & 2.92 & 3.40 & 3.02 & 1.96 & 2.47 & 0.24 & -3.18 & -3.09 & -2.27 & -1.67 & 3.37 & 1.87 & 0.73 \\
\hline \multirow{2}{*}{ Bragança } & MPI-hist & 28.01 & 27.29 & 24.05 & 23.54 & 21.07 & 18.02 & 19.44 & 18.73 & 20.07 & 23.16 & 24.63 & 26.32 & 22.8 \\
\hline & Differ. & 2.54 & 3.07 & 2.50 & 1.78 & 2.23 & 0.33 & -3.36 & -3.44 & -2.30 & -1.92 & 2.93 & 1.46 & 0.46 \\
\hline Porto & Differ. & 2.91 & 3.28 & 2.90 & 1.58 & 2.28 & 0.01 & -3.09 & -3.11 & -2.37 & -2.06 & 3.15 & 1.82 & 0.59 \\
\hline \multirow{2}{*}{ Aveiro } & MPI-hist & 27.70 & 27.27 & 24.42 & 24.62 & 21.82 & 18.51 & 19.68 & 18.57 & 20.01 & 23.05 & 24.29 & 25.71 & 22.95 \\
\hline & Differ. & 3.02 & 3.28 & 3.12 & 1.56 & 2.45 & -0.05 & -3.07 & -2.86 & -2.44 & -1.85 & 3.16 & 1.89 & 0.67 \\
\hline \multirow{2}{*}{ Viseu } & MPI-hist & 28.29 & 27.81 & 24.63 & 24.70 & 21.57 & 18.07 & 19.23 & 18.13 & 19.56 & 22.92 & 24.52 & 26.03 & 22.93 \\
\hline & Differ. & 2.53 & 2.77 & 2.70 & 1.09 & 2.09 & -0.16 & -3.43 & -2.97 & -2.44 & -1.94 & 2.88 & 1.37 & 0.36 \\
\hline \multirow[b]{2}{*}{ Guarda } & MPI-hist & 28.00 & 27.41 & 24.18 & 24.09 & 21.09 & 17.90 & 19.06 & 18.00 & 19.27 & 22.48 & 24.04 & 25.71 & 22.58 \\
\hline & Differ. & 2.36 & 2.67 & 2.73 & 1.29 & 2.23 & -0.12 & -3.52 & -2.93 & -2.35 & -1.67 & 2.85 & 1.18 & 0.38 \\
\hline \multirow{2}{*}{ Coimbra } & MPI-hist & 28.09 & 27.64 & 24.55 & 24.73 & 21.45 & 17.88 & 18.83 & 17.63 & 19.08 & 22.45 & 24.08 & 25.62 & 22.65 \\
\hline & Differ. & 2.29 & 2.45 & 2.51 & 0.81 & 1.94 & -0.33 & -3.33 & -2.72 & -2.50 & -1.98 & 2.69 & 1.08 & 0.23 \\
\hline Castelo & MPI-hist & 27.97 & 27.66 & 24.55 & 24.85 & 21.56 & 18.05 & 18.84 & 17.66 & 18.98 & 22.09 & 23.72 & 25.32 & 22.58 \\
\hline \multirow{2}{*}{ Leiria } & MPI-hist & 27.98 & 27.66 & 24.65 & 24.98 & 21.59 & 17.94 & 18.71 & 17.48 & 18.96 & 22.34 & 23.98 & 25.40 & 22.61 \\
\hline & Differ. & 2.27 & 2.34 & 2.48 & 0.69 & 1.90 & -0.47 & -3.10 & -2.52 & -2.56 & -1.93 & 2.53 & 1.08 & 0.21 \\
\hline \multirow{2}{*}{ Santarém } & MPI-hist & 27.84 & 27.65 & 24.69 & 25.24 & 21.80 & 18.01 & 18.60 & 17.35 & 18.79 & 22.01 & 23.70 & 25.09 & 22.54 \\
\hline & Differ. & 2.13 & 2.20 & 2.39 & 0.63 & 1.82 & -0.42 & -2.92 & -2.30 & -2.60 & -1.71 & 2.41 & 0.90 & 0.20 \\
\hline \multirow{2}{*}{ Lisboa } & MPI-hist & 27.67 & 27.70 & 24.81 & 25.68 & 22.20 & 18.19 & 18.50 & 17.26 & 18.67 & 21.84 & 23.60 & 24.85 & 22.55 \\
\hline & Differ. & 2.12 & 2.13 & 2.38 & 0.56 & 1.60 & -0.37 & -2.59 & -2.00 & -2.58 & -1.55 & 2.14 & 0.77 & 0.20 \\
\hline \multirow{2}{*}{ Portalegre } & MPI-hist & 27.85 & 27.71 & 24.69 & 25.18 & 21.99 & 18.28 & 18.72 & 17.63 & 18.88 & 21.85 & 23.61 & 25.08 & 22.60 \\
\hline & Differ. & 2.07 & 2.23 & 2.47 & 0.99 & 1.95 & -0.31 & -2.83 & -2.37 & -2.52 & -1.24 & 2.62 & 0.90 & 0.32 \\
\hline
\end{tabular}


Table 7. Cont

\begin{tabular}{|c|c|c|c|c|c|c|c|c|c|c|c|c|c|c|}
\hline \multirow{2}{*}{\multicolumn{2}{|c|}{ CITIES }} & \multicolumn{13}{|c|}{ Months } \\
\hline & & January & February & March & April & May & June & July & August & September & October & November & December & Year \\
\hline \multirow{2}{*}{ Évora } & MPI-hist & 27.82 & 27.93 & 25.00 & 25.95 & 22.74 & 18.57 & 18.68 & 17.46 & 18.66 & 21.68 & 23.60 & 24.84 & 22.72 \\
\hline & Differ. & 1.95 & 2.14 & 2.35 & 0.86 & 1.54 & -0.28 & -2.51 & -2.00 & -2.48 & -1.13 & 2.31 & 0.72 & 0.28 \\
\hline \multirow{2}{*}{ Setúbal } & MPI-hist & 27.68 & 27.77 & 24.87 & 25.84 & 22.43 & 18.32 & 18.56 & 17.28 & 18.57 & 21.75 & 23.55 & 24.81 & 22.59 \\
\hline & Differ. & 2.06 & 2.10 & 2.36 & 0.62 & 1.53 & -0.32 & -2.56 & -1.95 & -2.49 & -1.41 & 2.13 & 0.69 & 0.22 \\
\hline \multirow{2}{*}{ Beja } & MPI-hist & 27.90 & 28.24 & 25.30 & 26.52 & 23.41 & 18.76 & 18.72 & 17.40 & 18.52 & 21.53 & 23.68 & 24.79 & 22.87 \\
\hline & Differ. & 1.90 & 2.04 & 2.25 & 0.86 & 1.27 & -0.02 & -2.34 & -1.75 & -2.35 & -0.95 & 2.07 & 0.56 & 0.28 \\
\hline \multirow{2}{*}{ Faro } & MPI-hist & 25.92 & 25.54 & 22.85 & 24.38 & 20.09 & 16.15 & 15.34 & 14.81 & 16.20 & 20.54 & 22.88 & 23.67 & 20.67 \\
\hline & Differ. & 2.40 & 2.72 & 2.33 & 0.06 & 0.57 & -1.53 & -1.81 & -1.86 & -1.75 & -2.61 & 0.74 & 0.06 & -0.07 \\
\hline Lugo & Differ. & 2.62 & 3.34 & 2.57 & 2.09 & 1.78 & 0.32 & -2.52 & -3.86 & -2.18 & -2.23 & 3.41 & 2.04 & 0.59 \\
\hline \multirow[b]{2}{*}{ Bilbao } & MPI-hist & 28.06 & 27.20 & 23.83 & 22.25 & 20.51 & 18.97 & 20.63 & 20.53 & 21.55 & 23.67 & 25.14 & 26.84 & 23.25 \\
\hline & Differ. & 2.43 & 3.69 & 2.81 & 3.46 & 3.40 & 0.89 & -2.85 & -3.92 & -2.46 & -1.38 & 2.57 & 2.20 & 0.88 \\
\hline \multirow{2}{*}{ Zaragoza } & MPI-hist & 28.96 & 27.62 & 24.35 & 23.59 & 21.21 & 18.02 & 17.38 & 18.25 & 19.81 & 23.74 & 25.72 & 27.67 & 23.01 \\
\hline & Differ. & 1.81 & 3.35 & 2.89 & 1.94 & 1.46 & -1.25 & -2.00 & -4.05 & -2.70 & -2.19 & 3.00 & 1.36 & 0.28 \\
\hline \multirow[b]{2}{*}{ Barcelona } & MPI-hist & 28.74 & 27.28 & 24.44 & 22.80 & 20.70 & 17.94 & 17.16 & 18.08 & 19.90 & 23.42 & 25.65 & 27.12 & 22.75 \\
\hline & Differ. & 1.78 & 3.50 & 3.09 & 2.18 & 1.89 & -0.76 & -1.93 & -3.80 & -2.89 & -2.25 & 2.53 & 1.71 & 0.40 \\
\hline \multirow{2}{*}{ Madrid } & MPI-hist & 27.97 & 27.59 & 24.44 & 23.94 & 21.40 & 18.48 & 19.38 & 18.59 & 19.72 & 21.91 & 23.72 & 25.25 & 22.67 \\
\hline & Differ. & 1.88 & 2.56 & 2.70 & 2.20 & 2.75 & 0.26 & -3.67 & -3.01 & -3.09 & -0.85 & 2.70 & 1.24 & 0.46 \\
\hline Valencia & MPI-hist & 28.36 & 27.00 & 23.99 & 23.30 & 20.44 & 17.19 & 16.65 & 16.62 & 18.35 & 21.74 & 24.53 & 25.38 & 21.94 \\
\hline \multirow[b]{2}{*}{ Murcia } & MPI-hist & 27.92 & 26.89 & 24.08 & 24.17 & 20.66 & 17.15 & 16.84 & 16.03 & 17.60 & 21.06 & 23.83 & 24.38 & 21.69 \\
\hline & Differ. & 1.47 & 2.97 & 2.83 & 1.44 & 1.89 & -1.04 & -2.50 & -1.64 & -2.14 & -1.77 & 1.79 & 0.88 & 0.33 \\
\hline \multirow{2}{*}{ Sevilla } & MPI-hist & 26.49 & 25.84 & 22.98 & 24.26 & 20.19 & 16.52 & 15.70 & 15.07 & 16.40 & 20.51 & 23.06 & 23.88 & 20.88 \\
\hline & Differ. & 2.04 & 2.77 & 2.46 & 0.46 & 0.90 & -1.61 & -2.11 & -1.81 & -1.70 & -2.37 & 1.05 & 0.13 & 0.00 \\
\hline
\end{tabular}


Table 8. Monthly and annual averaged wind shear 0-6 km for each city and the difference between the future and the historical climates. Significant differences ( $t$-student $5 \%)$ are shown in red (positive) and blue (negative).

\begin{tabular}{|c|c|c|c|c|c|c|c|c|c|c|c|c|c|c|}
\hline \multirow{2}{*}{\multicolumn{2}{|c|}{ CITIES }} & \multicolumn{13}{|c|}{ Months } \\
\hline & & January & February & March & April & May & June & July & August & September & October & November & December & Year \\
\hline \multirow{2}{*}{ Braga } & MPI-hist & 17.05 & 16.73 & 15.42 & 14.83 & 13.51 & 12.27 & 13.71 & 12.74 & 12.56 & 14.16 & 14.82 & 15.98 & 14.47 \\
\hline & Differ. & 1.41 & 1.34 & 0.81 & 1.03 & 1.60 & 0.99 & -1.10 & -1.22 & -0.98 & -1.75 & 1.35 & 0.64 & 0.33 \\
\hline $\begin{array}{l}\text { Viana do } \\
\text { Castelo }\end{array}$ & Differ. & 1.34 & 1.44 & 1.03 & 0.91 & 1.64 & 1.01 & -0.25 & -0.37 & -0.46 & -1.46 & 1.52 & 0.65 & 0.58 \\
\hline \multirow{2}{*}{ Vila Real } & MPI-hist & 17.31 & 16.73 & 15.59 & 15.15 & 13.43 & 12.72 & 13.78 & 12.72 & 12.76 & 14.29 & 15.06 & 15.98 & 14.62 \\
\hline & Differ. & 1.58 & 1.66 & 0.99 & 0.73 & 1.75 & 0.50 & -1.09 & -1.07 & -0.94 & -1.66 & 1.37 & 1.13 & 0.40 \\
\hline \multirow{2}{*}{ Bragança } & MPI-hist & 18.98 & 18.55 & 16.40 & 15.54 & 13.77 & 12.47 & 12.86 & 12.29 & 13.06 & 15.22 & 16.49 & 17.83 & 15.27 \\
\hline & Differ. & 1.08 & 1.30 & 0.95 & 0.95 & 1.14 & -0.10 & -0.90 & -1.57 & -1.53 & -1.87 & 1.11 & 0.87 & 0.11 \\
\hline Porto & Differ. & 1.84 & 1.65 & 1.28 & 1.06 & 1.86 & 0.64 & -1.11 & -1.18 & -1.02 & -1.60 & 1.53 & 1.02 & 0.49 \\
\hline \multirow{2}{*}{ Aveiro } & MPI-hist & 14.90 & 14.56 & 14.08 & 13.74 & 12.55 & 12.48 & 13.68 & 12.22 & 11.93 & 12.74 & 13.19 & 13.80 & 13.32 \\
\hline & Differ. & 1.36 & 1.08 & 0.91 & 0.93 & 2.12 & 0.65 & -0.64 & -0.31 & -0.77 & -1.16 & 1.03 & 0.41 & 0.46 \\
\hline \multirow{2}{*}{ Viseu } & MPI-hist & 18.30 & 17.72 & 16.20 & 15.87 & 14.03 & 13.30 & 14.51 & 13.18 & 13.12 & 14.53 & 15.41 & 16.85 & 15.24 \\
\hline & Differ. & 1.58 & 1.93 & 1.53 & 0.79 & 2.00 & 0.64 & -1.02 & -0.74 & -1.26 & -1.61 & 1.67 & 1.25 & 0.55 \\
\hline \multirow{2}{*}{ Guarda } & MPI-hist & 17.81 & 17.06 & 15.91 & 15.52 & 13.83 & 13.12 & 14.70 & 13.37 & 12.99 & 13.74 & 14.77 & 16.06 & 14.90 \\
\hline & Differ. & 0.73 & 1.67 & 0.92 & 0.99 & 2.11 & 0.89 & -0.99 & -0.58 & -1.31 & -1.05 & 1.33 & 0.63 & 0.44 \\
\hline \multirow{2}{*}{ Coimbra } & MPI-hist & 16.75 & 16.37 & 15.72 & 15.61 & 14.28 & 13.63 & 15.46 & 14.12 & 13.28 & 13.68 & 14.11 & 15.11 & 14.84 \\
\hline & Differ. & 1.37 & 1.71 & 1.25 & 1.08 & 2.39 & 0.87 & -1.30 & -0.55 & -1.04 & -1.14 & 1.37 & 0.78 & 0.56 \\
\hline $\begin{array}{l}\text { Castelo } \\
\text { Branco }\end{array}$ & MPI-hist & 16.83 & 16.46 & 15.81 & 15.72 & 14.17 & 14.19 & 16.05 & 14.61 & 13.70 & 14.15 & 14.40 & 15.11 & 15.10 \\
\hline \multirow[b]{2}{*}{ Leiria } & MPI-hist & 16.03 & 15.73 & 15.19 & 15.19 & 13.89 & 13.55 & 15.52 & 14.07 & 12.89 & 13.26 & 13.59 & 14.50 & 14.45 \\
\hline & Differ. & 1.62 & 1.48 & 1.25 & 0.93 & 2.47 & 0.94 & -1.18 & -0.17 & -0.77 & -1.19 & 1.03 & 0.60 & 0.58 \\
\hline \multirow{2}{*}{ Santarém } & MPI-hist & 16.19 & 15.90 & 15.50 & 15.44 & 13.98 & 13.81 & 15.94 & 14.52 & 13.32 & 13.64 & 13.86 & 14.61 & 14.72 \\
\hline & Differ. & 1.73 & 1.84 & 1.47 & 1.00 & 2.40 & 1.11 & -0.79 & 0.15 & -0.86 & -0.77 & 1.63 & 1.46 & 0.86 \\
\hline \multirow{2}{*}{ Lisboa } & MPI-hist & 15.72 & 15.50 & 15.35 & 15.38 & 14.14 & 14.79 & 17.31 & 15.65 & 13.74 & 13.64 & 13.57 & 14.13 & 14.91 \\
\hline & Differ. & 1.82 & 2.03 & 1.64 & 1.19 & 2.79 & 1.65 & -0.04 & 0.85 & -0.45 & -0.48 & 1.81 & 1.52 & 1.19 \\
\hline \multirow{2}{*}{ Portalegre } & MPI-hist & 16.09 & 15.80 & 15.48 & 15.71 & 14.49 & 15.09 & 17.40 & 15.80 & 13.96 & 13.82 & 13.69 & 14.33 & 15.14 \\
\hline & Differ. & 1.62 & 1.92 & 1.60 & 1.39 & 2.65 & 1.55 & -0.16 & 0.54 & -0.67 & -0.55 & 1.84 & 1.49 & 1.09 \\
\hline \multirow{2}{*}{ Évora } & MPI-hist & 15.72 & 15.59 & 15.42 & 15.77 & 14.61 & 15.44 & 17.98 & 16.35 & 13.99 & 13.67 & 13.34 & 13.89 & 15.15 \\
\hline & Differ. & 1.55 & 1.80 & 1.62 & 1.40 & 2.79 & 2.01 & 0.15 & 0.97 & -0.40 & -0.25 & 1.88 & 1.38 & 1.24 \\
\hline
\end{tabular}


Table 8. Cont.

\begin{tabular}{|c|c|c|c|c|c|c|c|c|c|c|c|c|c|c|}
\hline \multirow{2}{*}{\multicolumn{2}{|c|}{ CITIES }} & \multicolumn{13}{|c|}{ Months } \\
\hline & & January & February & March & April & May & June & July & August & September & October & November & December & Year \\
\hline \multirow{2}{*}{ Setúbal } & \multirow{2}{*}{$\begin{array}{l}\text { MPI-hist } \\
\text { Differ. }\end{array}$} & 15.48 & 15.37 & 15.29 & 15.41 & 14.32 & 15.22 & 17.82 & 16.13 & 13.93 & 13.56 & 13.33 & 13.82 & 14.97 \\
\hline & & 1.79 & 1.98 & 1.68 & 1.34 & 2.90 & 1.87 & 0.13 & 1.01 & -0.37 & -0.24 & 1.85 & 1.43 & 1.28 \\
\hline \multirow{2}{*}{ Beja } & MPI-hist & 15.61 & 15.49 & 15.28 & 15.68 & 14.70 & 15.17 & 17.45 & 15.95 & 13.48 & 13.41 & 13.18 & 13.53 & 14.91 \\
\hline & Differ. & 1.38 & 1.71 & 1.49 & 1.46 & 2.69 & 2.16 & 0.24 & 1.11 & -0.26 & -0.19 & 1.64 & 1.23 & 1.22 \\
\hline \multirow{2}{*}{ Faro } & MPI-hist & 14.82 & 14.29 & 13.84 & 13.88 & 12.02 & 10.95 & 10.96 & 9.98 & 10.42 & 12.01 & 12.79 & 13.30 & 12.43 \\
\hline & Differ. & 1.76 & 2.25 & 1.64 & 0.73 & 1.21 & -0.63 & -0.46 & -0.31 & -1.15 & -1.38 & 0.99 & 1.12 & 0.47 \\
\hline \multirow{2}{*}{ Lugo } & MPI-hist & 19.20 & 18.25 & 16.47 & 15.40 & 13.92 & 13.69 & 14.41 & 13.98 & 14.14 & 15.68 & 16.45 & 17.91 & 15.78 \\
\hline & Differ. & 0.83 & 1.30 & 1.16 & 1.08 & 1.42 & 0.30 & 0.00 & -1.22 & -0.99 & -1.65 & 1.87 & 1.51 & 0.46 \\
\hline Bilbao & Differ. & 0.58 & 1.51 & 0.45 & 2.20 & 2.43 & 0.92 & -1.01 & -2.09 & -1.27 & -1.08 & 0.50 & 0.90 & 0.32 \\
\hline \multirow{2}{*}{ Zaragoza } & MPI-hist & 17.38 & 16.60 & 15.11 & 13.54 & 12.30 & 11.41 & 11.05 & 11.44 & 12.30 & 13.97 & 15.47 & 16.50 & 13.91 \\
\hline & Differ. & 0.86 & 1.57 & 0.99 & 1.66 & 1.43 & -0.44 & -0.51 & -2.05 & -1.70 & -1.53 & 1.35 & 1.43 & 0.25 \\
\hline \multirow{2}{*}{ Barcelona } & MPI-hist & 19.87 & 19.09 & 17.58 & 16.11 & 14.53 & 13.18 & 12.64 & 13.36 & 14.47 & 16.31 & 17.66 & 18.69 & 16.11 \\
\hline & Differ. & 0.92 & 1.82 & 1.78 & 1.73 & 1.61 & -0.48 & -1.18 & -2.86 & -2.43 & -1.91 & 1.54 & 1.85 & 0.19 \\
\hline \multirow{2}{*}{ Madrid } & MPI-hist & 17.55 & 17.29 & 16.11 & 15.27 & 13.91 & 13.99 & 15.91 & 14.64 & 13.85 & 13.95 & 14.70 & 15.71 & 15.23 \\
\hline & Differ. & 0.71 & 1.06 & 1.07 & 1.65 & 2.78 & 1.33 & -1.31 & -0.67 & -1.59 & -0.60 & 1.34 & 0.96 & 0.56 \\
\hline \multirow{2}{*}{ Valencia } & MPI-hist & 19.19 & 18.05 & 16.62 & 15.35 & 13.45 & 11.88 & 11.43 & 11.40 & 12.77 & 14.76 & 16.88 & 17.47 & 14.92 \\
\hline & Differ. & 1.29 & 2.00 & 1.50 & 1.52 & 1.94 & -0.54 & -1.21 & -1.92 & -2.29 & -1.63 & 1.28 & 1.72 & 0.30 \\
\hline Murcia & MPI-hist & 18.16 & 17.17 & 15.79 & 15.22 & 13.14 & 11.02 & 11.08 & 10.38 & 11.75 & 13.30 & 15.11 & 15.85 & 13.98 \\
\hline \multirow[b]{2}{*}{ Sevilla } & MPI-hist & 15.95 & 15.18 & 14.34 & 14.11 & 11.99 & 10.63 & 10.57 & 9.70 & 10.33 & 12.41 & 13.57 & 14.28 & 12.74 \\
\hline & Differ. & 1.39 & 1.89 & 1.22 & 0.64 & 1.09 & -0.67 & -0.79 & -0.64 & -1.29 & -1.52 & 1.10 & 1.05 & 0.28 \\
\hline
\end{tabular}


Table 9. Monthly and annual averaged storm-relative helicity (SRH) 0-3 km for each city and the difference between the future and the historical climates. Significant differences $(t$-student $5 \%$ ) are shown in red (positive) and blue (negative).

\begin{tabular}{|c|c|c|c|c|c|c|c|c|c|c|c|c|c|c|}
\hline \multirow{2}{*}{\multicolumn{2}{|c|}{ CITIES }} & \multicolumn{13}{|c|}{ Months } \\
\hline & & January & February & March & April & May & June & July & August & September & October & November & December & Year \\
\hline \multirow{2}{*}{ Braga } & MPI-hist & 8.09 & 6.06 & 3.98 & 2.29 & -1.43 & -0.41 & -3.87 & -4.81 & -2.19 & 3.27 & 5.07 & 5.80 & 1.80 \\
\hline & Differ. & -1.46 & -0.47 & 0.39 & -3.32 & -2.62 & -3.86 & 0.42 & 2.11 & -0.63 & -4.37 & -0.70 & 1.11 & -1.11 \\
\hline $\begin{array}{l}\text { Viana do } \\
\text { Castelo }\end{array}$ & Differ. & -1.49 & -1.11 & 1.29 & -0.47 & 1.96 & 4.56 & 5.29 & 8.43 & 3.71 & -0.35 & 0.70 & 2.14 & 2.08 \\
\hline \multirow{2}{*}{ Vila Real } & MPI-hist & 2.63 & 1.29 & 2.41 & 1.21 & -1.26 & 4.27 & 7.19 & 4.10 & 2.53 & 1.08 & 0.62 & 0.98 & 2.26 \\
\hline & Differ. & 0.14 & 0.67 & 1.11 & -1.79 & 3.01 & 3.12 & 4.91 & 8.10 & 1.10 & -0.14 & 1.94 & 3.20 & 2.14 \\
\hline \multirow{2}{*}{ Bragança } & MPI-hist & 24.74 & 20.26 & 17.01 & 8.18 & 4.15 & 1.42 & 0.38 & -1.53 & 2.94 & 10.62 & 18.88 & 20.11 & 10.55 \\
\hline & Differ. & -1.06 & -1.60 & 0.21 & -0.05 & -1.53 & -0.79 & 4.75 & 5.95 & -0.27 & -4.68 & -2.01 & 2.43 & 0.14 \\
\hline Porto & Differ. & 1.55 & 1.35 & 2.45 & -3.23 & -0.84 & -1.67 & 2.00 & 2.24 & 0.19 & -4.39 & 0.73 & 4.21 & 0.39 \\
\hline \multirow{2}{*}{ Aveiro } & MPI-hist & 2.99 & 2.27 & 3.39 & 2.92 & 0.34 & 1.19 & -3.07 & -2.77 & 0.16 & 3.27 & 3.38 & 1.37 & 1.27 \\
\hline & Differ. & 1.34 & 1.22 & 3.11 & -2.06 & 0.39 & -2.23 & -0.35 & -1.53 & 0.15 & -1.16 & 2.11 & 4.94 & 0.50 \\
\hline \multirow{2}{*}{ Viseu } & MPI-hist & 11.69 & 6.07 & 5.37 & 3.30 & 1.79 & 3.22 & 2.50 & 2.32 & 4.55 & 6.47 & 9.60 & 10.42 & 5.61 \\
\hline & Differ. & -2.62 & 2.03 & 1.48 & -0.69 & 1.67 & -1.54 & 2.28 & 2.67 & -2.30 & -1.80 & -1.59 & 2.20 & 0.15 \\
\hline \multirow{2}{*}{ Guarda } & MPI-hist & -3.04 & -4.44 & -1.17 & -0.51 & 0.59 & 2.59 & 2.28 & 2.17 & 0.50 & 0.62 & -0.98 & -4.22 & -0.45 \\
\hline & Differ. & -1.40 & -0.51 & -0.62 & -1.71 & 0.02 & 0.90 & 6.44 & 5.95 & 2.06 & -2.18 & -3.28 & -0.63 & 0.44 \\
\hline \multirow{2}{*}{ Coimbra } & MPI-hist & -0.70 & -1.85 & -0.87 & -0.92 & -0.52 & 0.99 & -4.88 & -3.99 & -2.33 & -0.08 & -2.16 & -3.46 & -1.74 \\
\hline & Differ. & -3.54 & 0.29 & -0.30 & -1.61 & -0.70 & -3.60 & 5.25 & 3.68 & -0.07 & -1.74 & -1.71 & 0.20 & -0.31 \\
\hline $\begin{array}{l}\text { Castelo } \\
\text { Branco }\end{array}$ & MPI-hist & 4.23 & 2.78 & 4.95 & 3.77 & 5.46 & 10.40 & 12.31 & 10.99 & 8.61 & 8.40 & 6.63 & 4.24 & 6.92 \\
\hline \multirow[b]{2}{*}{ Leiria } & MPI-hist & 0.91 & 1.06 & 1.34 & 1.29 & 3.08 & 8.20 & 4.75 & 2.48 & 1.19 & 1.67 & -0.55 & -0.69 & 2.06 \\
\hline & Differ. & -1.86 & -0.16 & 0.20 & -0.45 & 0.54 & -0.10 & 10.42 & 6.97 & 1.41 & -0.86 & -1.13 & 0.40 & 1.31 \\
\hline \multirow{2}{*}{ Santarém } & MPI-hist & 1.44 & 1.39 & 3.19 & 2.46 & 6.21 & 12.52 & 9.79 & 8.21 & 5.54 & 5.73 & 4.89 & 2.66 & 5.35 \\
\hline & Differ. & 1.95 & 1.81 & 2.49 & 1.43 & 2.07 & 1.96 & 10.02 & 6.06 & 3.97 & 0.61 & -0.47 & 4.27 & 3.04 \\
\hline \multirow{2}{*}{ Lisboa } & MPI-hist & 2.03 & 2.02 & 4.21 & 3.02 & 6.53 & 16.04 & 18.39 & 14.20 & 7.53 & 7.14 & 5.99 & 3.62 & 7.60 \\
\hline & Differ. & 3.46 & 2.15 & 3.32 & 2.06 & 3.88 & 7.50 & 12.50 & 11.30 & 7.92 & 1.82 & -0.62 & 4.07 & 4.98 \\
\hline \multirow{2}{*}{ Portalegre } & MPI-hist & 4.48 & 4.34 & 5.53 & 4.61 & 7.09 & 14.88 & 18.99 & 16.03 & 10.94 & 9.07 & 6.38 & 4.95 & 8.97 \\
\hline & Differ. & 2.37 & 1.02 & 4.27 & 2.13 & 5.00 & 5.17 & 6.31 & 8.52 & 4.83 & 2.42 & 0.16 & 3.16 & 3.81 \\
\hline \multirow{2}{*}{ Évora } & MPI-hist & 3.73 & 2.64 & 3.49 & 2.11 & 5.93 & 11.88 & 16.27 & 12.21 & 7.22 & 6.87 & 5.90 & 3.77 & 6.87 \\
\hline & Differ. & 1.12 & 0.64 & 4.05 & 2.09 & 3.14 & 5.18 & 3.07 & 10.84 & 7.46 & 2.50 & -1.75 & 2.12 & 3.39 \\
\hline
\end{tabular}


Table 9. Cont

\begin{tabular}{|c|c|c|c|c|c|c|c|c|c|c|c|c|c|c|}
\hline \multirow{2}{*}{\multicolumn{2}{|c|}{ CITIES }} & \multicolumn{13}{|c|}{ Months } \\
\hline & & January & February & March & April & May & June & July & August & September & October & November & December & Year \\
\hline \multirow{2}{*}{ Setúbal } & MPI-hist & 2.54 & 1.91 & 3.31 & 1.86 & 5.13 & 12.68 & 14.95 & 10.71 & 4.99 & 5.99 & 5.11 & 2.96 & 6.04 \\
\hline & Differ. & 1.87 & 0.34 & 2.56 & 1.89 & 3.62 & 6.09 & 7.17 & 10.42 & 8.61 & 1.92 & -1.49 & 2.78 & 3.84 \\
\hline \multirow{2}{*}{ Beja } & MPI-hist & 3.12 & 1.82 & 1.40 & -0.11 & 1.21 & 2.01 & -0.71 & 0.27 & 0.21 & 2.80 & 3.35 & 2.96 & 1.53 \\
\hline & Differ. & -1.86 & -0.96 & 1.80 & 0.21 & 1.40 & -1.28 & 0.33 & 5.92 & 4.22 & 0.90 & -3.37 & -1.07 & 0.54 \\
\hline \multirow[b]{2}{*}{ Faro } & MPI-hist & 2.97 & 1.48 & 4.23 & 2.10 & 2.22 & 2.97 & 2.80 & 2.61 & 2.17 & 3.00 & 4.99 & 3.81 & 2.95 \\
\hline & Differ. & 2.71 & 1.73 & 0.84 & -1.39 & -0.19 & 0.32 & 0.65 & 0.71 & 0.96 & -0.11 & 0.02 & 0.89 & 0.59 \\
\hline \multirow{2}{*}{ Lugo } & MPI-hist & 34.58 & 26.61 & 22.51 & 15.19 & 9.85 & 10.31 & 10.76 & 7.62 & 9.55 & 21.28 & 30.54 & 29.88 & 19.03 \\
\hline & Differ. & 1.98 & 4.01 & 3.28 & -2.41 & 0.11 & 0.16 & 9.05 & 10.66 & 2.18 & -5.30 & -0.96 & 8.17 & 2.60 \\
\hline Bilbao & Differ. & -4.60 & -3.00 & 0.40 & -1.78 & -1.22 & -2.02 & -4.03 & -1.10 & -2.51 & -3.99 & -3.72 & -9.37 & -3.09 \\
\hline \multirow{2}{*}{ Zaragoza } & MPI-hist & -3.49 & -2.77 & -2.60 & -1.42 & -0.28 & 4.19 & 7.79 & 5.63 & 1.02 & -1.49 & -3.00 & -4.37 & -0.05 \\
\hline & Differ. & -1.89 & -0.43 & 0.72 & 1.15 & 3.08 & 3.79 & 5.32 & 5.52 & 2.36 & 0.46 & -2.41 & -0.33 & 1.46 \\
\hline \multirow[b]{2}{*}{ Barcelona } & MPI-hist & 5.94 & 4.23 & 2.26 & 3.87 & 0.31 & 2.67 & 2.13 & 1.65 & 4.79 & 3.38 & 6.00 & 7.24 & 1.00 \\
\hline & Differ. & -1.99 & -2.96 & -0.69 & -4.60 & 2.41 & -0.98 & 3.87 & 1.05 & -0.84 & 0.44 & 0.36 & -1.70 & -0.44 \\
\hline \multirow{2}{*}{ Madrid } & MPI-hist & 4.16 & 2.21 & 4.31 & 3.31 & 4.63 & 7.98 & 10.96 & 7.56 & 5.46 & 5.53 & 4.98 & 1.71 & 5.25 \\
\hline & Differ. & 3.00 & 0.84 & 2.52 & 1.25 & 4.06 & 3.74 & 2.04 & 6.10 & 2.98 & 1.35 & 0.02 & 4.46 & 2.72 \\
\hline \multirow[b]{2}{*}{ Valencia } & MPI-hist & -6.95 & -4.00 & -1.78 & -2.98 & -1.13 & 3.10 & 5.56 & 3.14 & 2.68 & -2.83 & -2.56 & -7.48 & -1.26 \\
\hline & Differ. & 1.68 & -2.11 & -0.91 & 2.43 & 3.02 & 0.94 & 3.96 & 4.04 & 0.81 & 3.42 & -0.72 & 1.74 & 1.56 \\
\hline Murcia & MPI-hist & -6.11 & -6.05 & -4.23 & -3.72 & -1.35 & 3.88 & 4.44 & 4.58 & 2.04 & -3.45 & -4.71 & -6.43 & -1.74 \\
\hline \multirow{2}{*}{ Sevilla } & MPI-hist & 6.16 & 3.58 & 5.57 & 3.60 & 3.25 & 6.98 & 7.64 & 6.78 & 5.31 & 5.54 & 8.23 & 6.33 & 5.76 \\
\hline & Differ. & 4.27 & 2.14 & 1.86 & -0.49 & 1.91 & 0.89 & 0.77 & 0.84 & 1.29 & -0.14 & -1.23 & 2.49 & 1.22 \\
\hline
\end{tabular}




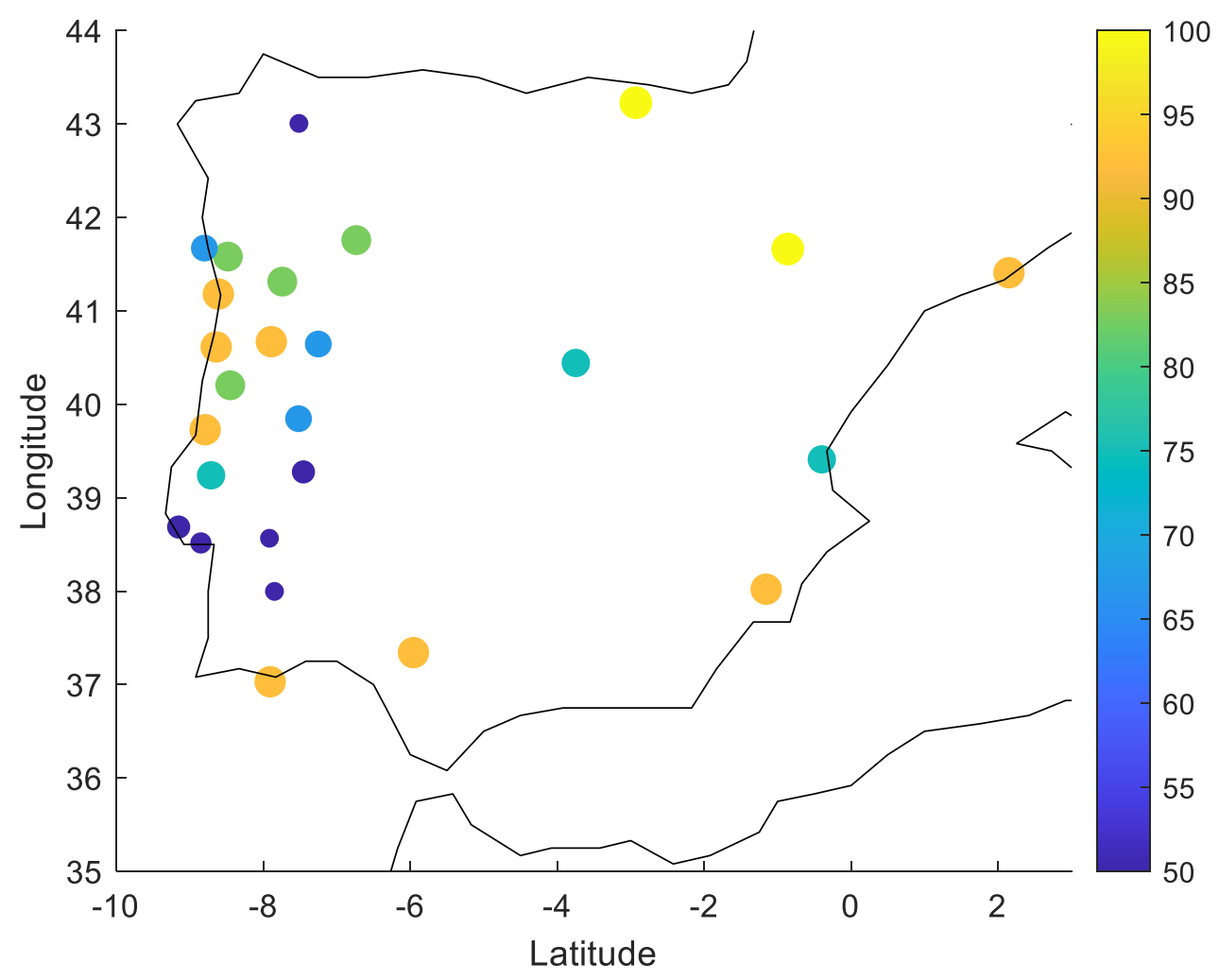

Figure 4. Average percentage of time in one year that near surface wind speed differences, future minus historic, are negative.

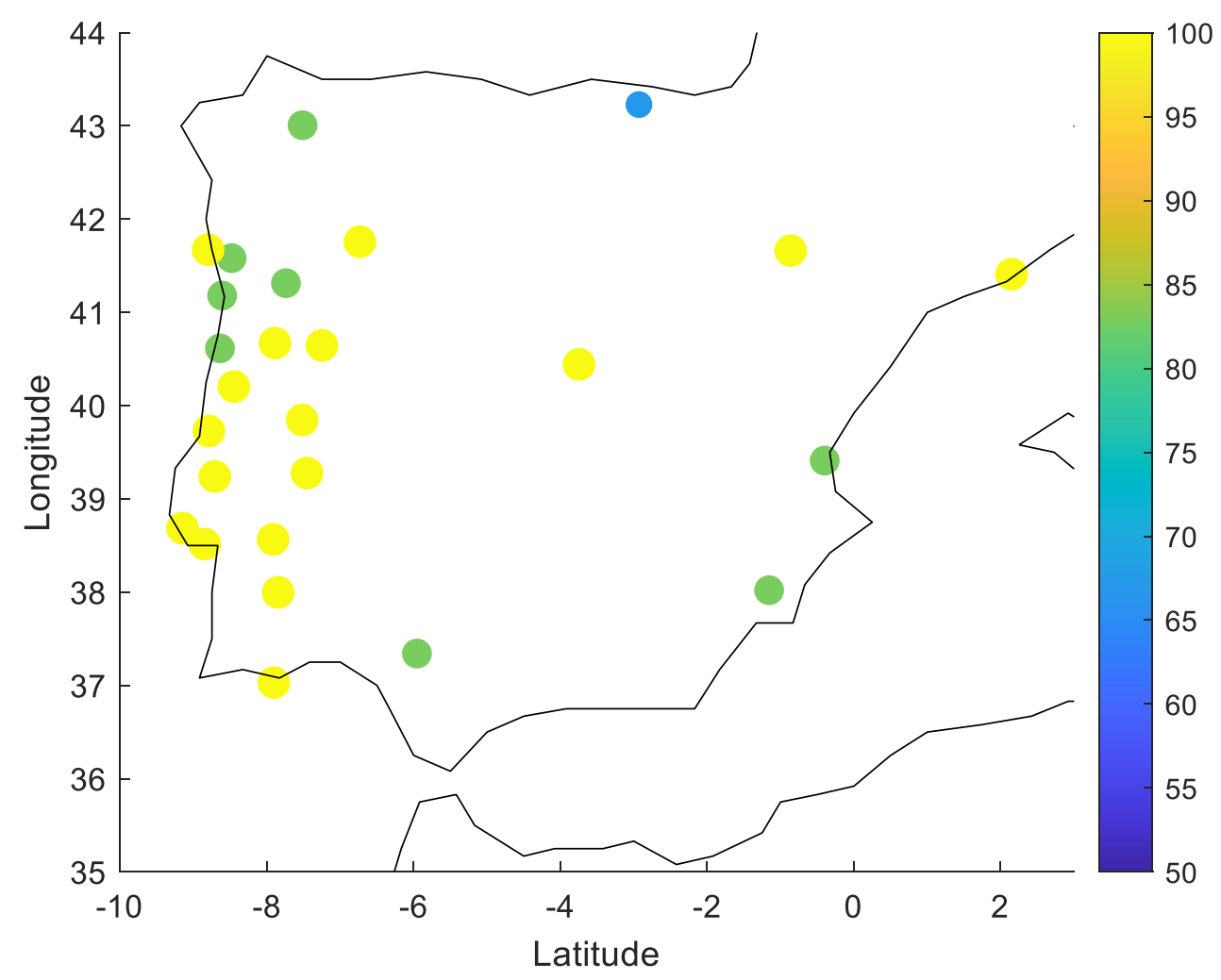

Figure 5. Average percentage of time for the May-October period that $850 \mathrm{hPa}$ wind speed differences, future minus historic, are negative. 


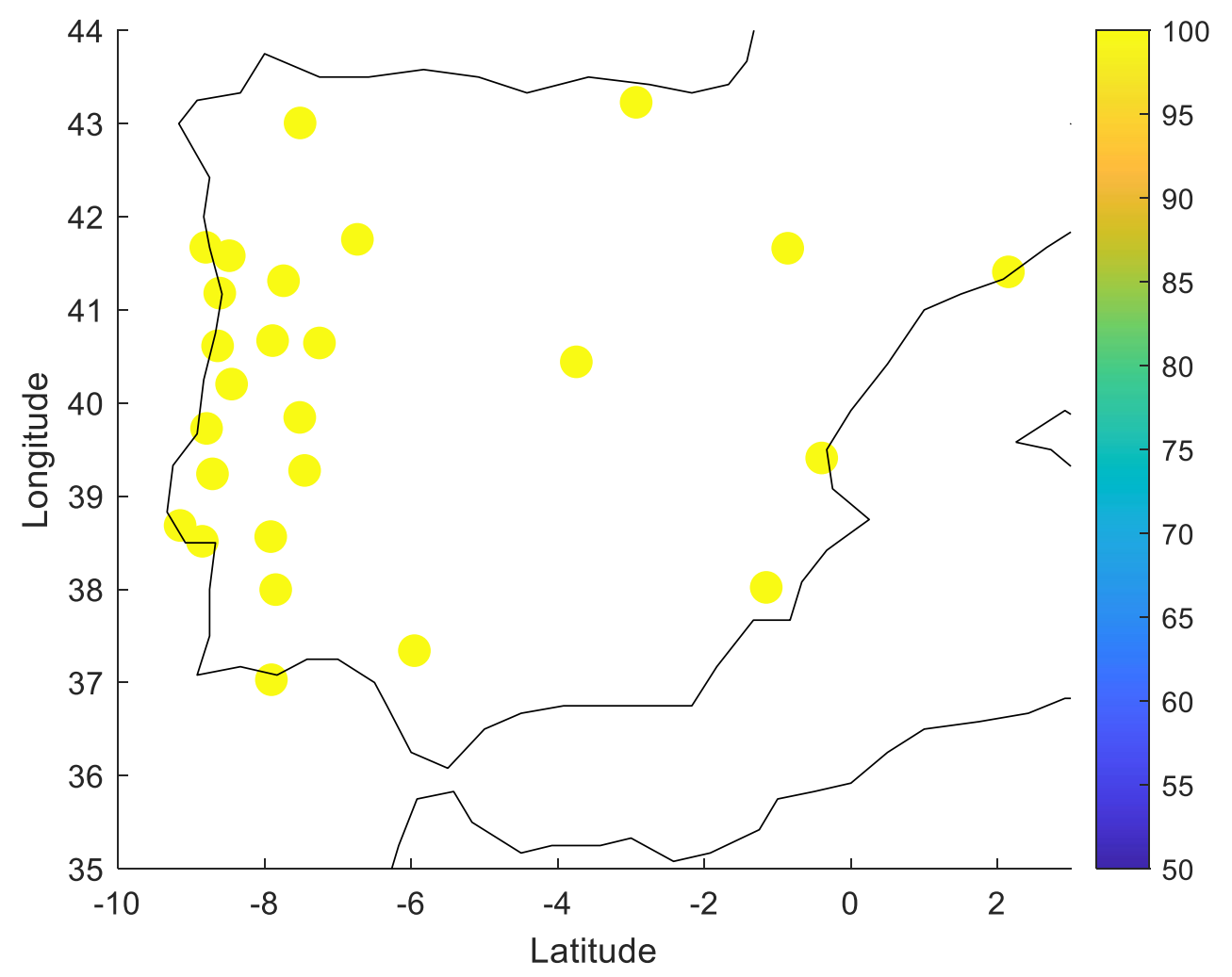

Figure 6. Average percentage of time for the July-September period that $300 \mathrm{hPa}$ wind speed differences, future minus historic, are negative.

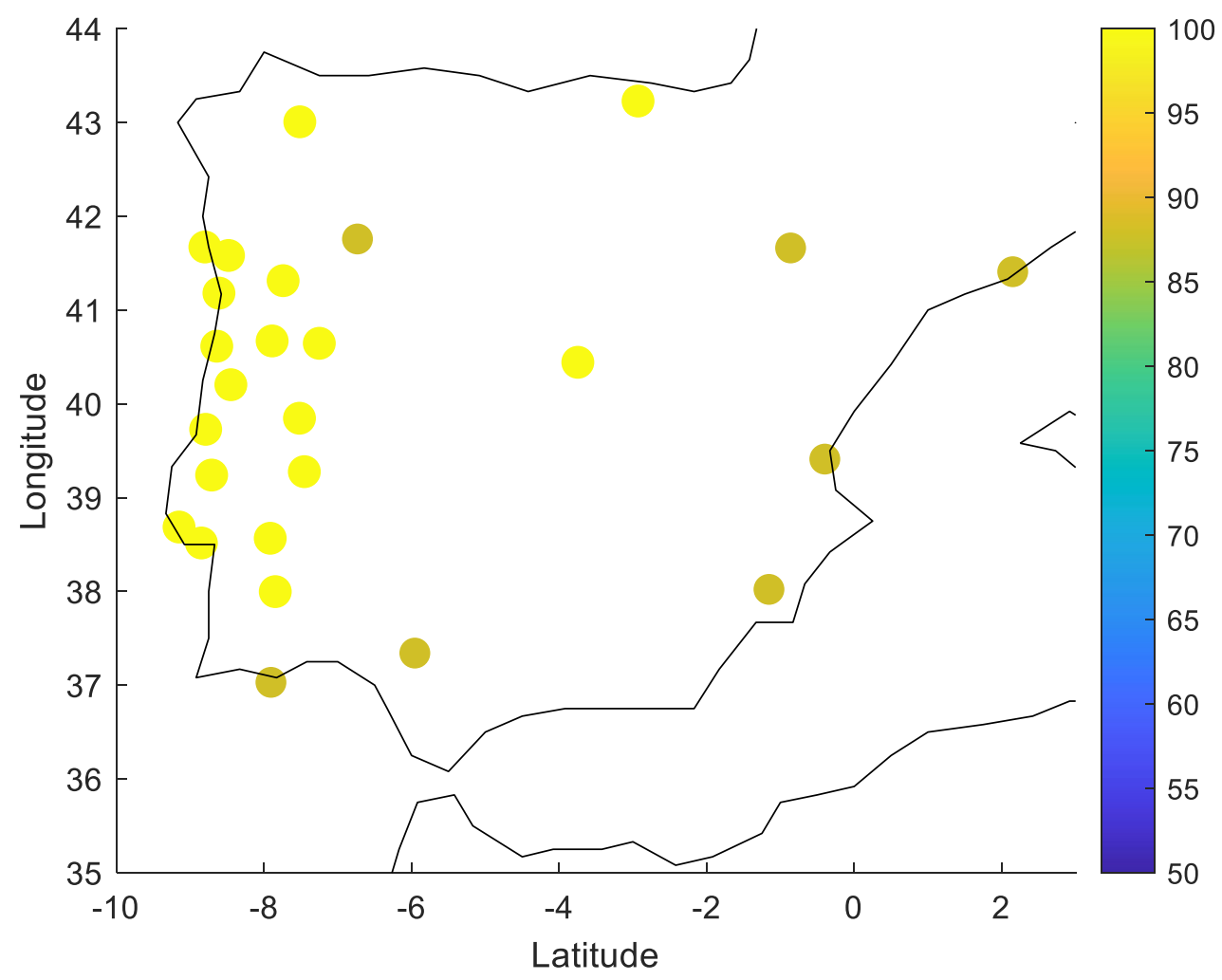

Figure 7. Average percentage of time for the November-June period that wind shear 0-6 km differences, future minus historic, are positive. 


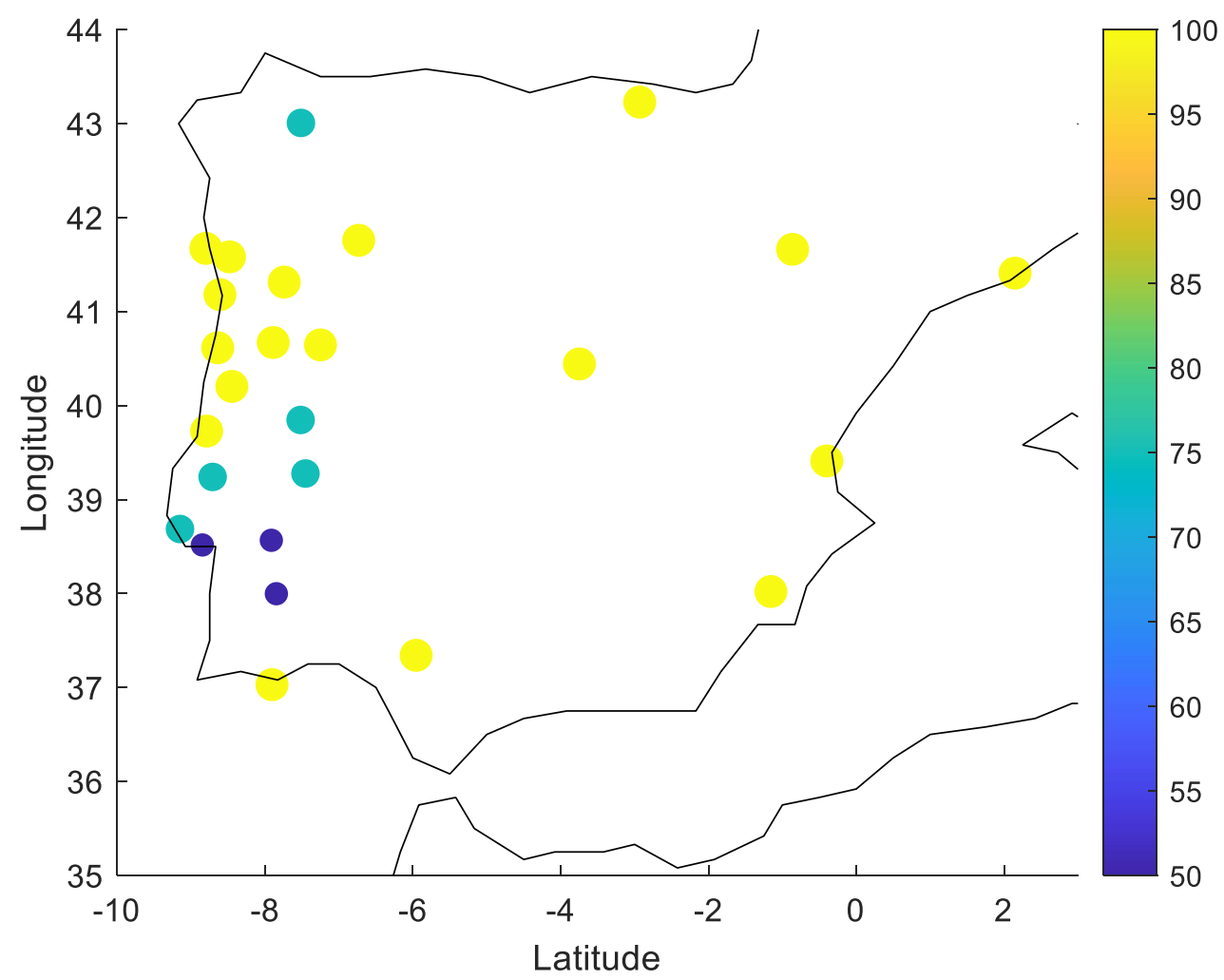

Figure 8. Average percentage of time for the July-October period that wind shear 0-6 km differences, future minus historic, are negative.

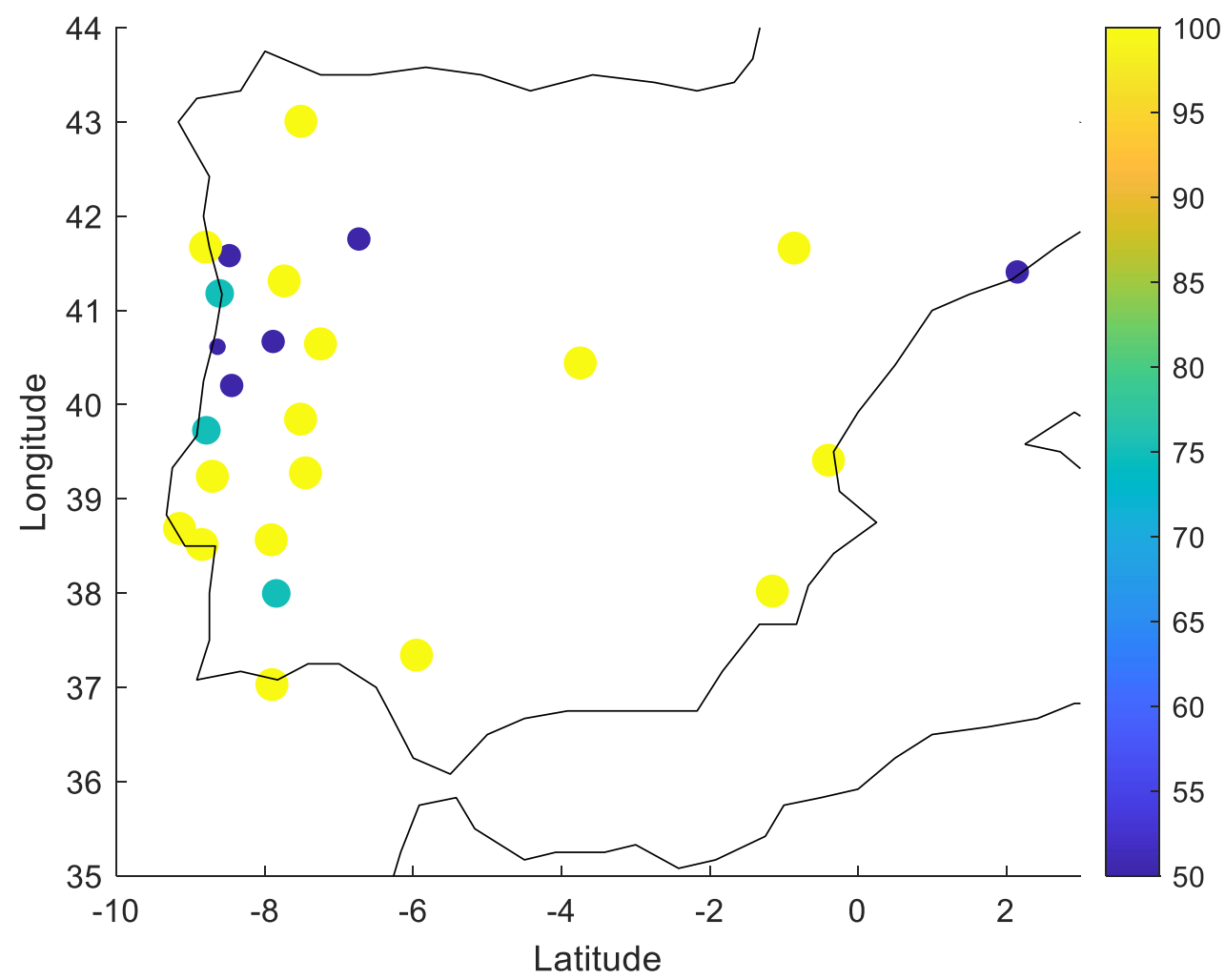

Figure 9. Average percentage of time for the June-September period that storm-relative helicity (SRH) 0-3 km differences, future minus historic, are positive. 
These changes broadly represent a reduction of wind speed in the lower troposphere during the year and in the upper troposphere only during summer. The latter also occurs for wind shear 0-6 km with a simultaneous increase of storm-relative helicity (SRH) $0-3 \mathrm{~km}$. These conditions are clearly a signature of climate change, particularly of tropospheric warming in the region and a shift towards a more tropical-like environment with more favourable conditions for convective storms during summer (i.e., more helicity and weaker horizontal winds and wind shear).

In Figure 10, the hodograph for Lisbon is represented as an illustration. The hodograph was calculated from 0 to $6 \mathrm{~km}$ (for every $1 \mathrm{~km}$ ). The hodographs for all cities are generally similar, (not shown). These show an increase of wind shear for the whole 0-6 km layers and an anticlockwise rotation of the wind between 5 and $10^{\circ}$.

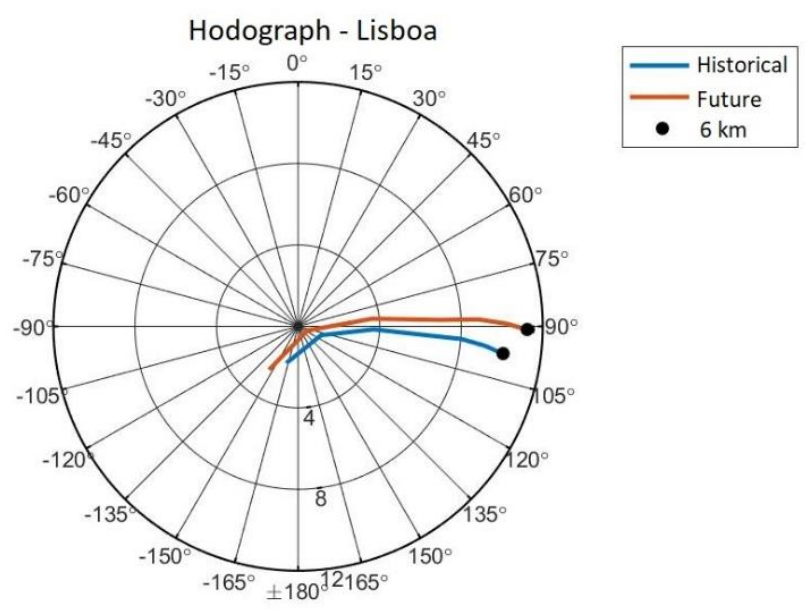

Figure 10. Hodograph for Lisboa, from 0 to $6 \mathrm{~km}$, for historical (blue line) and future (orange line) climates.

\subsection{Distributional Changes}

In this section, the statistical distribution of the variables is studied for both climates. The quantiles were calculated and their future minus historic difference is obtained. This facilitates the identification of changes across the whole range of distributions. This analysis is performed for annual and seasonal conditions.

\subsubsection{Wind at $850 \mathrm{hPa}$}

Figure 11 shows $850 \mathrm{hPa}$-wind speed quantile differences between future and historic climates for all cities, for both annual and seasonal conditions. For the former, the differences are generally negative across the distribution. Regarding the seasonal analysis, winter is the only period depicting nearly zero differences. Largest negative differences are observed for summer and autumn. These represent, for annual conditions, a reduction of wind speed in the future of about $5 \%$ or $0.5 \mathrm{~m} \mathrm{~s}^{-1}$, on average, for all cities with some dispersion across cities. This may represent a reduction of resources for wind energy applications. Extreme right quantiles are about $15 \mathrm{~m} \mathrm{~s}^{-1}$ for quantile $0.90,19 \mathrm{~m} \mathrm{~s}^{-1}$ for quantile 0.95 , and $25 \mathrm{~m} \mathrm{~s}^{-1}$ for quantile 0.99 . Maximum values are about $48 \mathrm{~m} \mathrm{~s}^{-1}$. 
(a) Diff

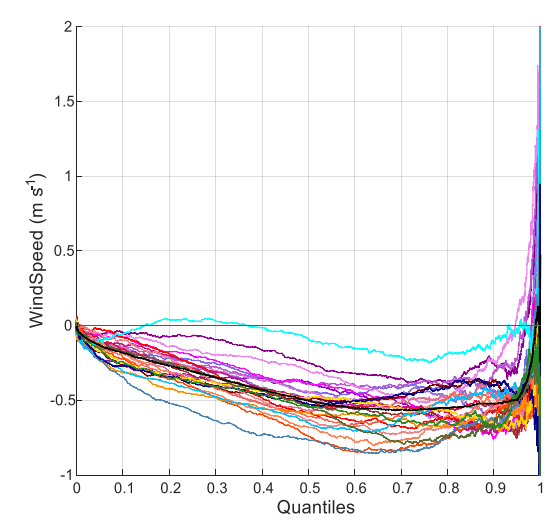

(b) $\operatorname{Diff}(\%)$

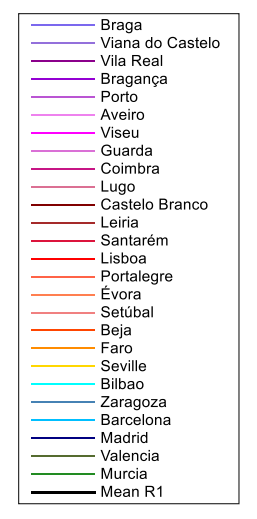

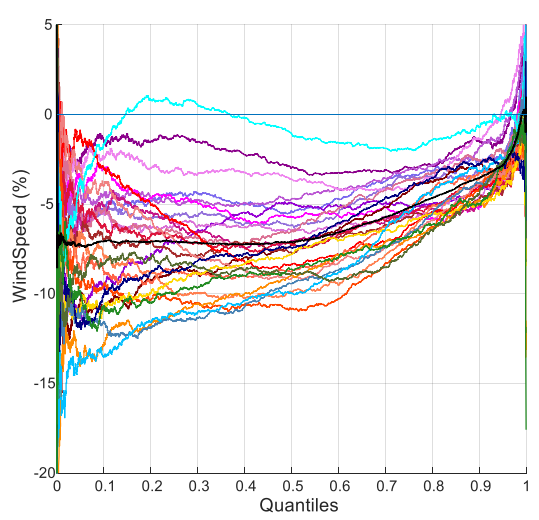

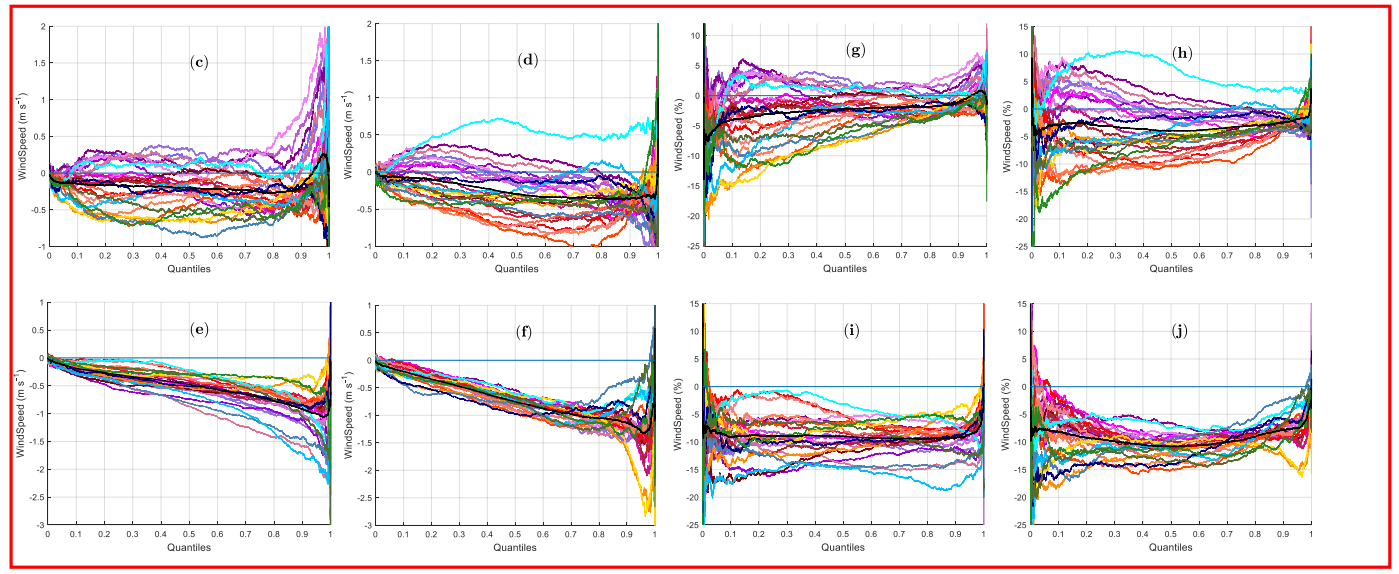

Figure 11. Differences (future-historic) for wind speed at $850 \mathrm{hPa}$ for annual and seasonal conditions, as a function of the quantiles of the corresponding empirical distributions. (a) Absolute differences (m $\mathrm{s}^{-1}$ ) and (b) relative percentual differences (\%). Seasonal absolute differences are also represented for (c) winter, (d) spring, (e) summer and (f) autumn, and Seasonal percentual differences for (g) winter, (h) spring, (i) summer and (j) autumn. The black line (Mean R1) refers to the mean of region 1 (total area).

\subsubsection{Wind at $300 \mathrm{hPa}$}

Figure 12 presents the quantile differences for wind speed at $300 \mathrm{hPa}$. For annual conditions, upper tropospheric wind speed changes have the opposite sign on the left and right parts of the distribution. For lower quantiles, there is a reduction of about $4 \%$ or $0.6 \mathrm{~m} \mathrm{~s}^{-1}$ in the future relative to the historic periods, whereas, for the quantiles above the median wind speed, it is expected to increase by $7 \%$ or $4 \mathrm{~m} \mathrm{~s}^{-1}$. For this variable, extreme right quantiles are about $38 \mathrm{~m} \mathrm{~s}^{-1}$ for quantile $0.90,45 \mathrm{~m}$ $\mathrm{s}^{-1}$ for quantile 0.95 , and $55 \mathrm{~m} \mathrm{~s}^{-1}$ for quantile 0.99 . Maximum values are about $75 \mathrm{~m} \mathrm{~s}^{-1}$. Concerning the seasonal conditions, autumn reveals a similar pattern to annual conditions. For winter and spring, differences are positive across the distribution, whereas for summer these are negative. The polar jet stream is strongest during winter and is typically identified at this pressure level when wind speed is over about $26 \mathrm{~m} \mathrm{~s}^{-1}$. The jet stream may reach wind speeds of about $130 \mathrm{~m} \mathrm{~s}^{-1}$ in its core. In this analysis, equatorward excursions of the polar jet stream in the region of study may be represented by simulated wind speeds at $300 \mathrm{hPa}$ above quantiles 0.6-0.7. Additionally, the position and intensity of the polar jet stream determine, to a great extent, the development of surface depressions and storm tracks. Therefore, the results found may represent a southward shift of the jet stream, an increase of its wind speed, or a more meandering jet as a result of climate change. It is beyond the scope of this paper to study these processes, but wind speed changes identified here may be related to these. These processes in the scope of climate change are fully discussed by some authors (e.g., [65-68]). 
(a) Diff

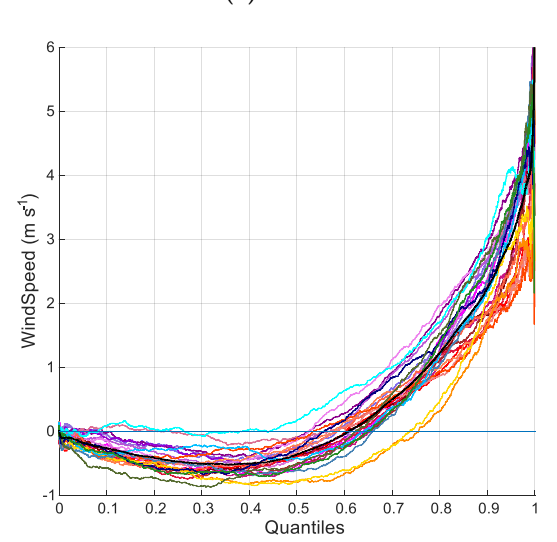

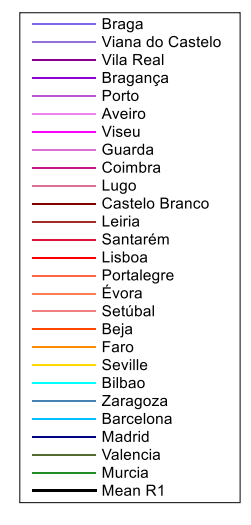

(b) Diff (\%)

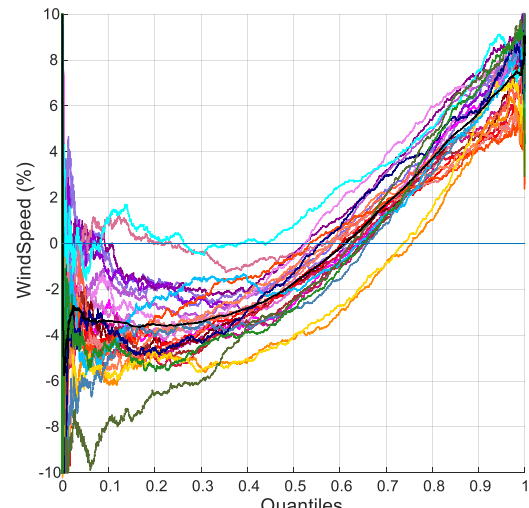

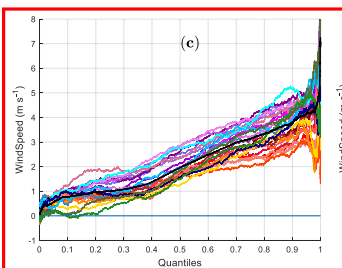

(e)

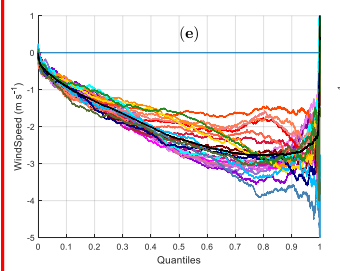

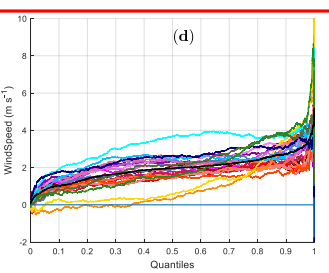

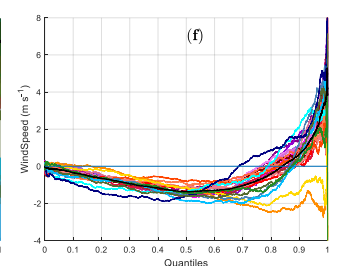

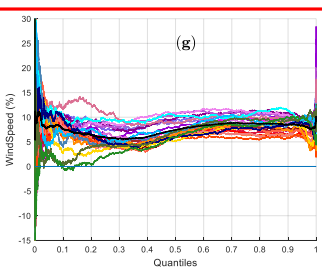
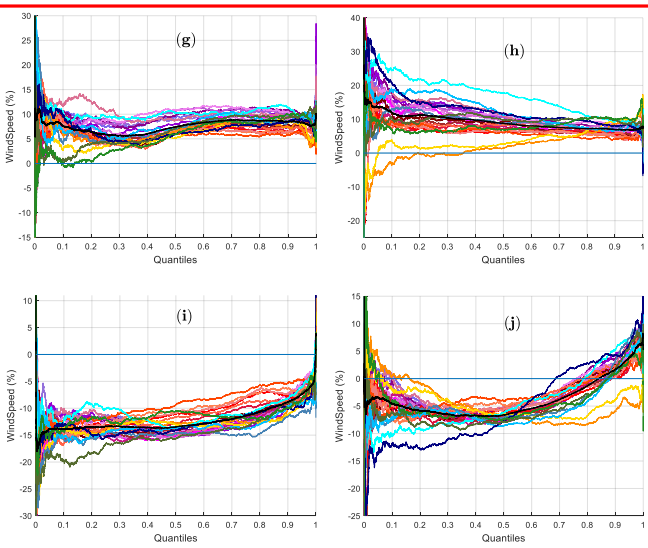

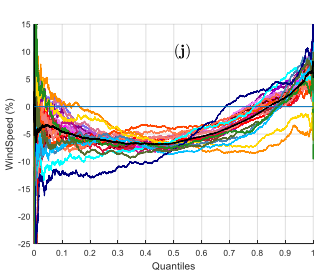

Figure 12. Differences (future-historic) for wind speed at $300 \mathrm{hPa}$ for annual and seasonal conditions, as a function of the quantiles of the corresponding empirical distributions. (a) Absolute differences (m $\mathrm{s}^{-1}$ ) and (b) relative percentual differences (\%). Seasonal absolute differences are also represented for (c) winter, (d) spring, (e) summer and (f) autumn, and Seasonal percentual differences for (g) winter, (h) spring, (i) summer and (j) autumn. The black line (Mean R1) refers to the mean of region 1 (total area).

\subsubsection{Wind Shear $(0-6 \mathrm{~km})$}

Figure 13 shows $0-6 \mathrm{~km}$ wind shear quantile differences between future and historic climates for all cities for annual and seasonal conditions. For the former, the differences represent an increase reduction of wind shear in the extreme right of the distribution in the future of about $5 \%$ or $1.5 \mathrm{~m} \mathrm{~s}^{-1}$ on average for all cities with some dispersion across cities.

For this variable, the extreme right quantiles are about $25 \mathrm{~m} \mathrm{~s}^{-1}$ for quantile $0.90,30 \mathrm{~m} \mathrm{~s}^{-1}$ for quantile 0.95 and $35 \mathrm{~m} \mathrm{~s}^{-1}$ for quantile 0.99 . Maximum values are about 51 to $75 \mathrm{~m} \mathrm{~s}^{-1}$. These extreme values are well within the range of reference values shown in Tables 2 and 3 for different types of storms/tornados. An increase of $5 \%$ in these extreme right quantiles means more frequent conditions for the development of intense storms in the region. For winter and spring, differences are positive across the distribution with increases of the order of $10-15 \%$ in the future relative to the historic climate. 
(a) Diff

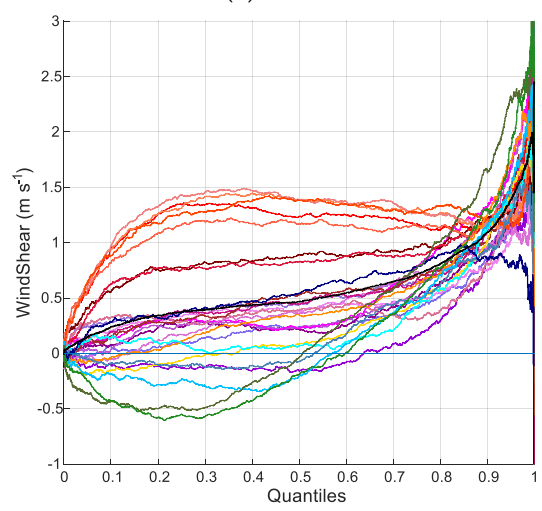

(b) Diff (\%)

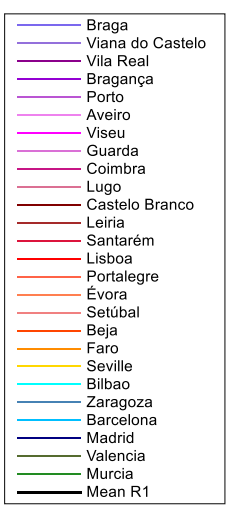

(b) $\operatorname{Diff}(\%)$
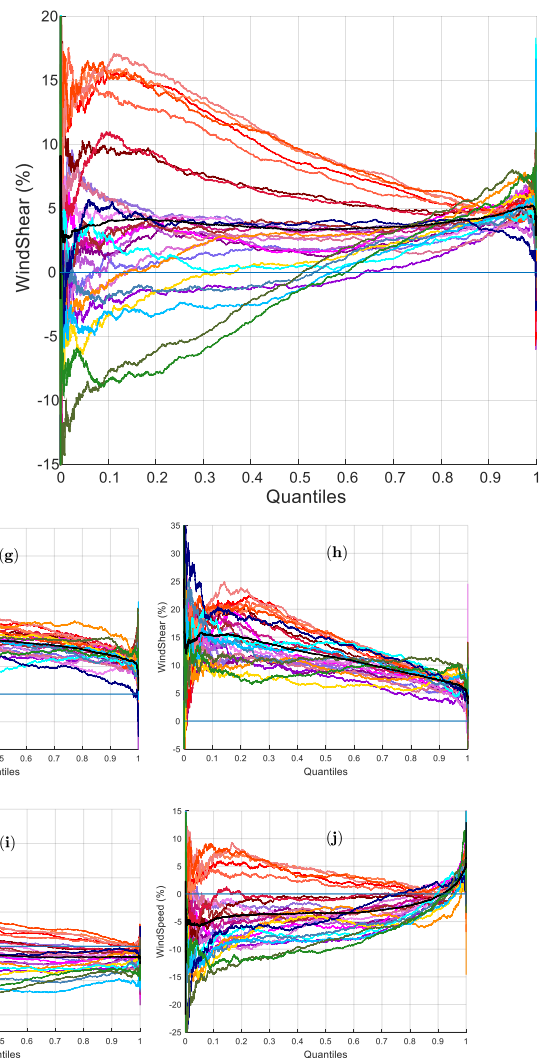

Figure 13. Differences (future-historic) for wind shear 0-6 km for annual and seasonal conditions, as a function of the quantiles of the corresponding empirical distributions. (a) Absolute differences ( $\mathrm{m} \mathrm{s}^{-1}$ ) and $(\mathbf{b})$ relative percentual differences $(\%)$. Seasonal absolute differences are also represented for (c) winter, (d) spring, (e) summer and (f) autumn, and Seasonal percentual differences for (g) winter, (h) spring, (i) summer and (j) autumn.The black line (Mean R1) refers to the mean of region 1 (total area).

\subsubsection{Helicity $(0-3 \mathrm{~km})$}

Figure 14 shows 0-3 km SRH quantile differences between future and historic climates for all cities for annual and seasonal conditions. For the former, differences represent a reduction of helicity in the future of about $-10 \%$ or $-5 \mathrm{~m}^{2} \mathrm{~s}^{2}$ for the left extreme tail and $+10 \%$ or $10 \mathrm{~m}^{2} \mathrm{~s}^{2}$ for the right extreme tail, on average, for all cities, with some dispersion across cities. For seasonal conditions, spring and summer are similar to annual, whereas, for autumn and winter, differences are less pronounced.

For this variable, only extreme positive values are relevant for right moving storms, since their extreme right quantiles are about $40 \mathrm{~m}^{2} \mathrm{~s}^{2}$ for quantile $0.90,60 \mathrm{~m}^{2} \mathrm{~s}^{2}$ for quantile 0.95 and $120 \mathrm{~m}^{2} \mathrm{~s}^{2}$ for quantile 0.99 . The maximum values across cities range from 275 to $744 \mathrm{~m}^{2} \mathrm{~s}^{2}$. These extreme values are well within the range of reference values shown in Tables $2-4$ for different types of storms/tornados. An increase of $10 \%$ in these extreme right quantiles means more frequent conditions for the development of intense storms in the region and, at least, F0 and F1 tornados. 


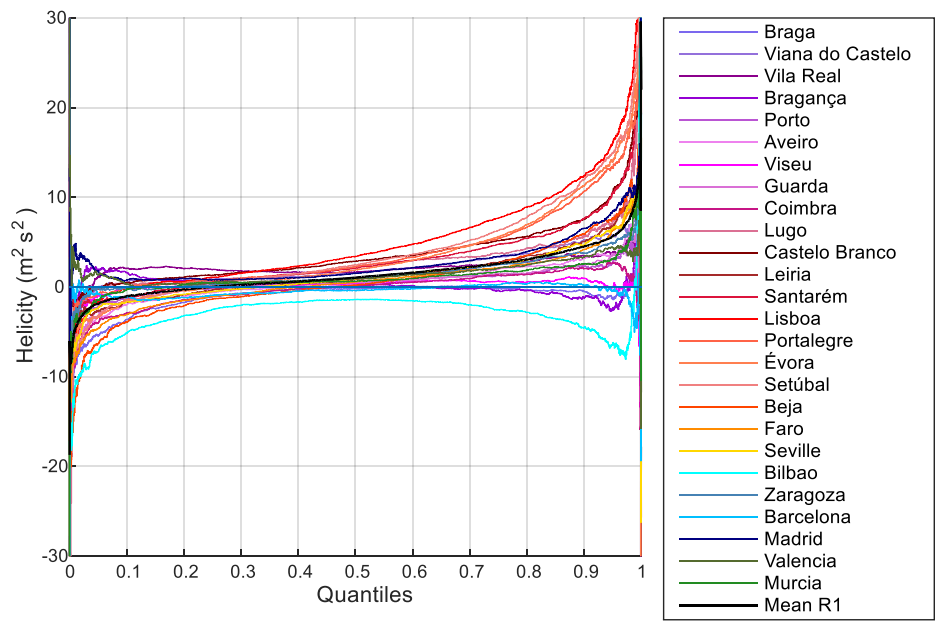

(a)

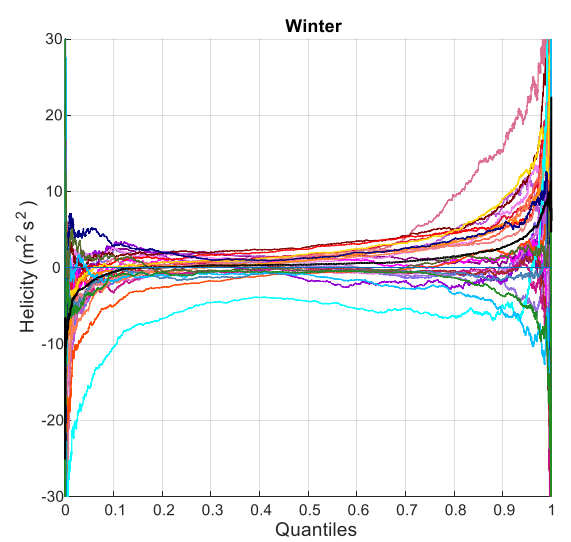

(b)

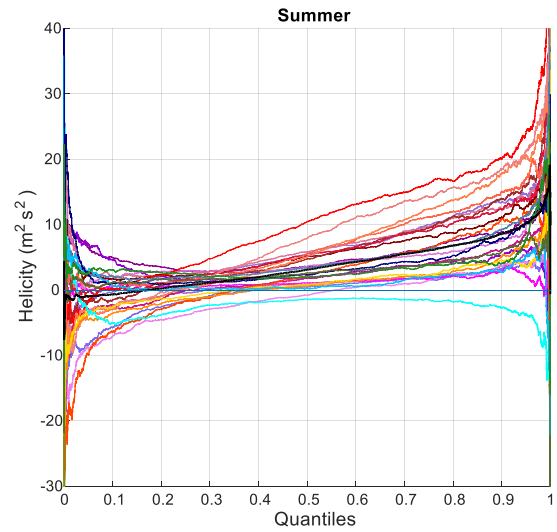

(d)

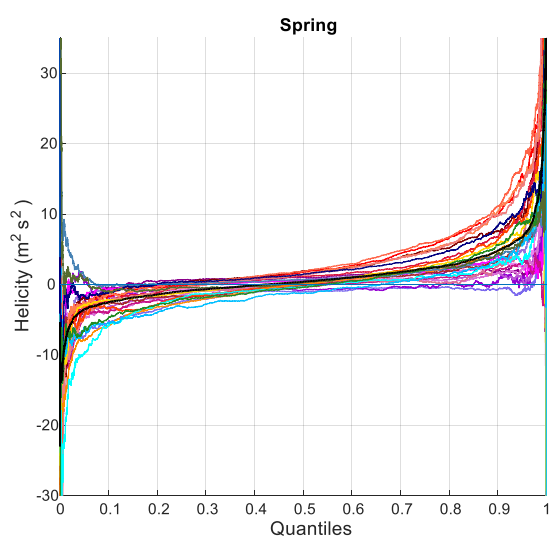

(c)

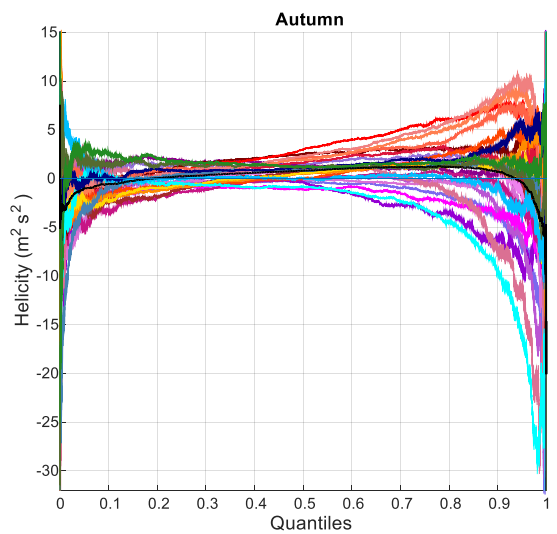

(e)

Figure 14. Absolute differences $\left(\mathrm{m}^{2} \mathrm{~s}^{2}\right.$, future-historic) for helicity $0-3 \mathrm{~km}$ for annual and seasonal conditions, as a function of the quantiles of the corresponding empirical distributions. The black line (Mean R1) refers to the mean of region 1 (total area). (a) annual, (b) winter, (c) spring, (d) summer, (e) winter. 


\subsection{Extreme Event Changes in Averages}

\subsubsection{Wind at $850 \mathrm{hPa}$}

Figure 15 displays a comparison between historical (blue) and future periods (red) in all cities, while taking into account the average duration of events (Figure 15a) and number of extreme events per year (Figure 15b), for the wind speed at $850 \mathrm{hPa}$. The average duration of extreme events identically decreases in all cities. As for the number of events, it markedly decreases in the future, maintaining the same pattern as in the historical period.

(a)

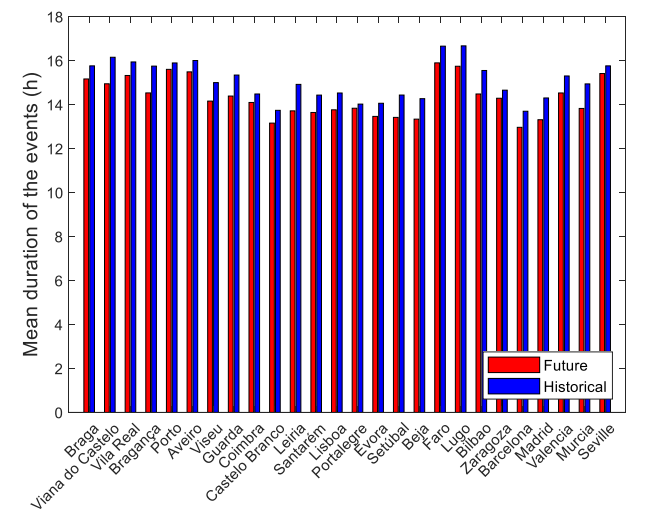

(b)

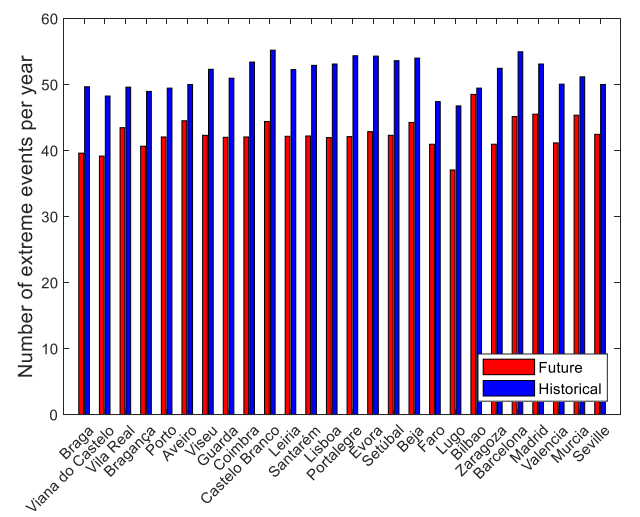

Figure 15. (a) Mean duration (hours) of the extreme events of the wind speed at $850 \mathrm{hPa}$ and (b) the number of extreme events per year, for both the historical (blue) and future (red) climates and all cities.

\subsubsection{Wind at $300 \mathrm{hPa}$}

Figure 16 represents the comparison graphs between historical (blue) and future periods (red) in all cities, while taking into account the average duration of events in hours (Figure 16a) and the number of extreme events per year (Figure 16b), for the wind speed at $300 \mathrm{hPa}$. There are some differences when comparing with the wind speed at $850 \mathrm{hPa}$. In this case, the average duration of extreme events increases sharply, while the number of extreme events increases in the north and decreases to the south.

(a)

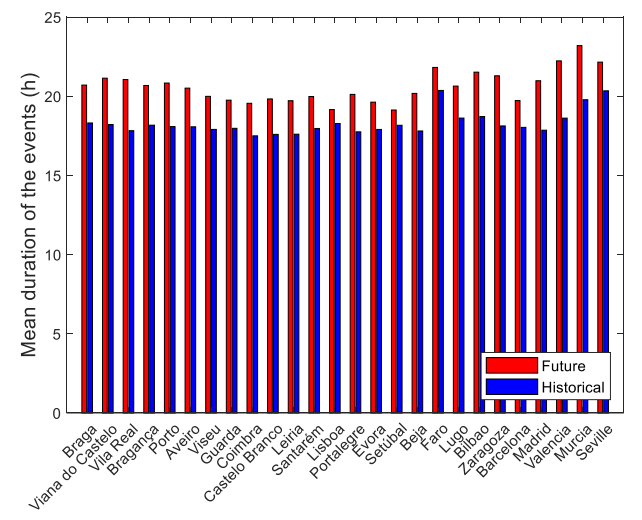

(b)

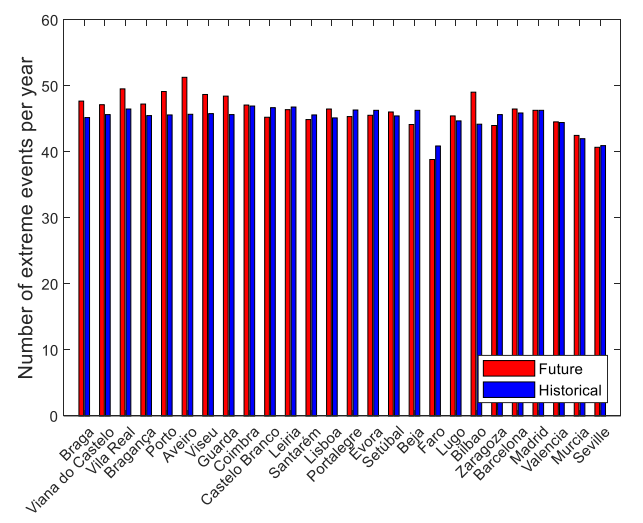

Figure 16. (a) Mean duration (hours) of extreme events of the wind speed at $300 \mathrm{hPa}$ and (b) the number of extreme events per year, for historical (blue) and future (red) climates and for all cities.

\subsubsection{Wind Shear $(0-6 \mathrm{~km})$}

Figure 17 shows the comparison graphs between historical (blue) and future periods (red) in all cities, while taking into account the average duration of events in hours (Figure 17a) and the number 
of extreme events per year (Figure 17b), for the intensity of the wind shear between 0 and $6 \mathrm{~km}$. For the intensity of the wind shear, once again it appears that the average duration of the extreme events increases in the future, in this case, mainly in the south. On the other hand, the number of extreme events per year increases in the future, except in the south (Faro, Valencia, Murcia, and Seville). The increase is more accentuated in central/southern Portugal.

(a)

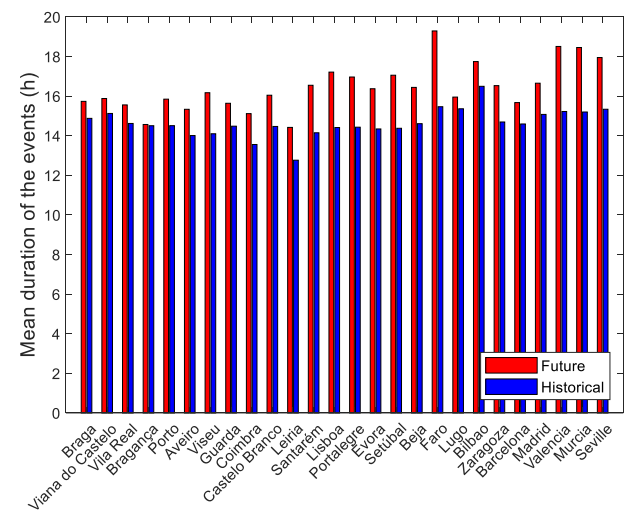

(b)

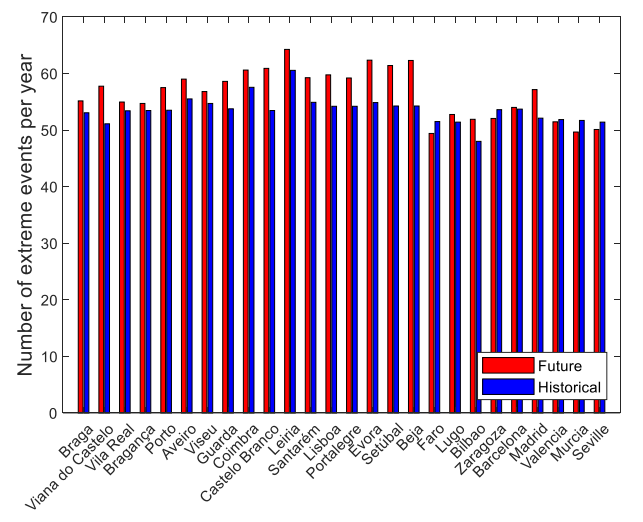

Figure 17. (a) Mean duration (hours) of the extreme events of the wind shear and (b) number of extreme events per year, for historical (blue) and future (red) climates for all cities.

\subsubsection{Helicity $(0-3 \mathrm{~km})$}

In the case of helicity (Figure 18), the effect of climate change is manifested by an increase in the mean event duration and the total number of events per year, emerging a north-south contrast in the latter index.

(a)

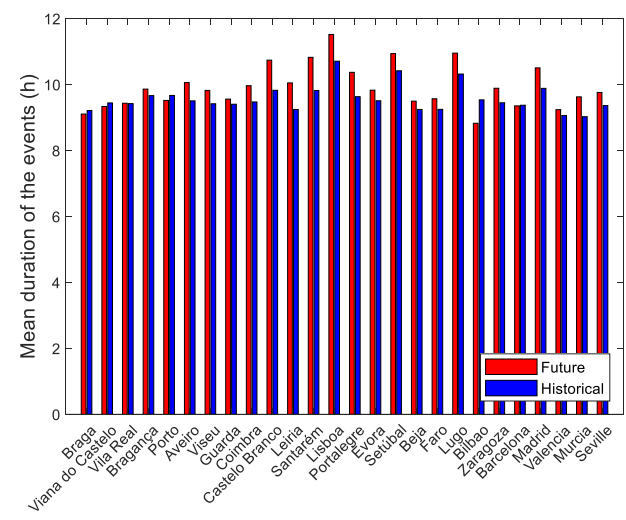

(b)

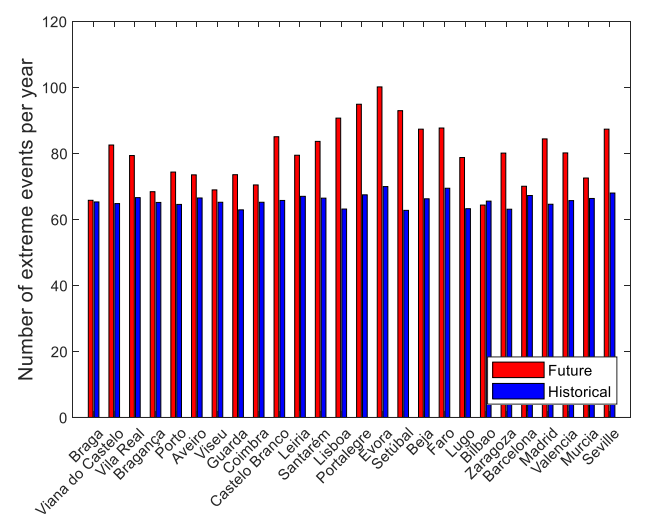

Figure 18. (a) Mean duration (hours) of the extreme events of the helicity and (b) number of extreme events per year, for historical (blue) and future (red) climates for all cities.

\subsection{Extreme Event Changes-Changes by Event Type}

\subsubsection{Wind at $850 \mathrm{hPa}$}

The wind speed shows that for these type of events, region, and season, when the difference between the historical and the future periods is statistically significant, its value is negative, indicating that the number of events that occurred in the past is greater than the number of events projected for the future (Figure 19). The cells are shown in the same layout as Figure 2. 


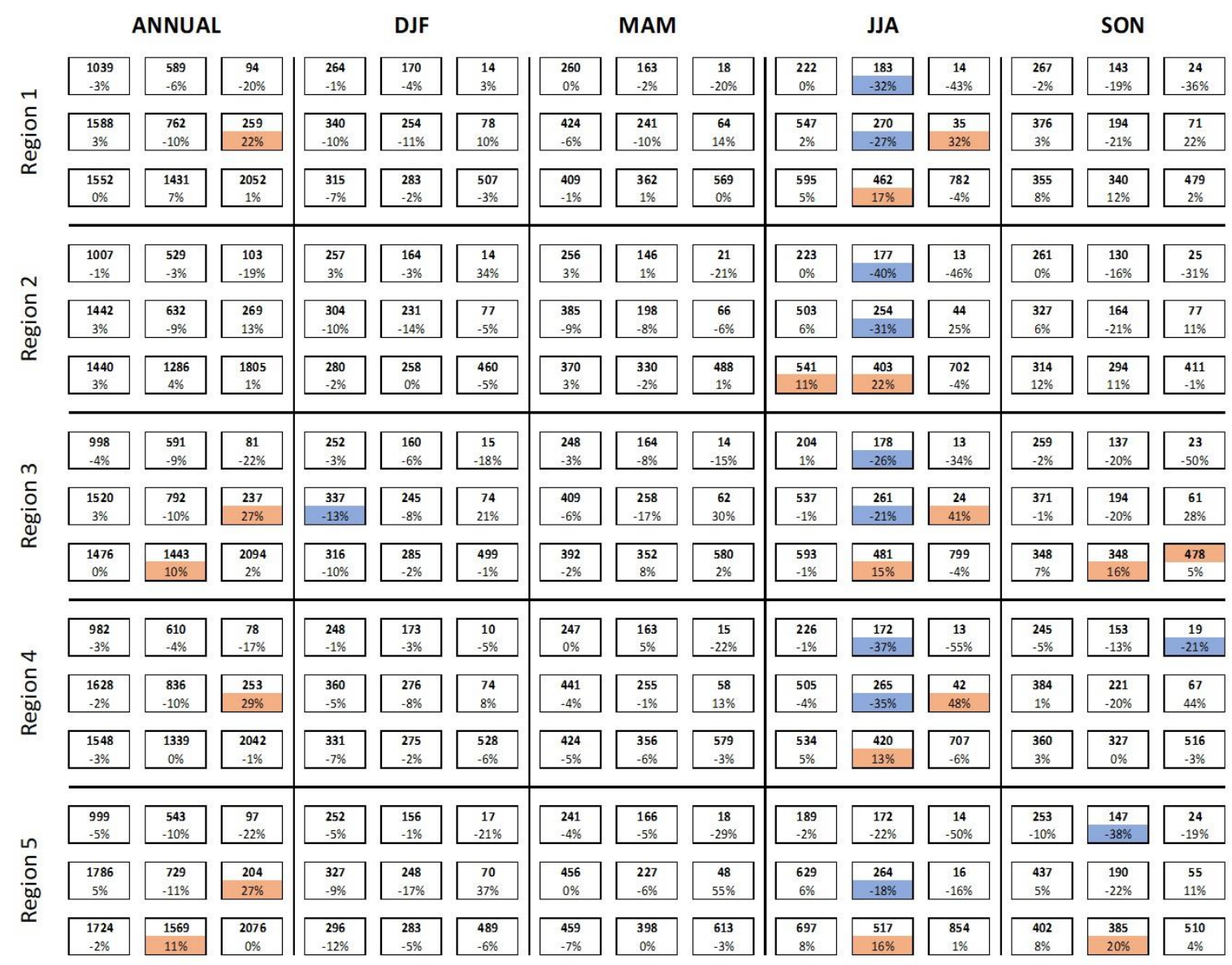

Figure 19. The number of wind speed events at $850 \mathrm{hPa}$ for each type of event, region and season. For the historical climate (1st line of the cell) and the difference between the future and historical (2nd line of the cell). Shaded values indicate significant differences ( $t$-student $5 \%$ ) positive (red) and negative (blue).

The types with the highest number of cells indicative of statistical significance are types 2, 3, 7, and 8. The types of events with the highest values are type 7 , followed by 8 , which will decrease the most in terms of their occurrence in the future. In annual conditions, it is projected an increase between $22 \%$ and $27 \%$ for the number of episodes of type 7 . This category represents episodes with mean intensity between $5 \mathrm{~m} \mathrm{~s}^{-1}\left(18 \mathrm{~km} \mathrm{~h}^{-1}\right)$ and $16 \mathrm{~m} \mathrm{~s}^{-1}\left(57.6 \mathrm{~km} \mathrm{~h}^{-1}\right)$ approx. and duration above $36 \mathrm{~h}$ (Table A2).

June, July, and August reveal the highest number of statistically significant differences. Region 3 is the one with the highest number of statistically significant differences, followed by region 4 and 5 . Therefore, it is generally expected that events of type 3 and 8 will occur less frequently, and events of type 2 and 7 will be more frequent in the future period. This is particularly clear for the summer months.

\subsubsection{Wind at $300 \mathrm{hPa}$}

The wind speed at $300 \mathrm{hPa}$ (Figure 20) shows that most statistically significant differences are positive, which suggests an increase in the number of extreme events for some regions, seasons and types in the future. In annual conditions, it is projected an increase between $49 \%$ and $60 \%$ for the number of episodes of type 9 . This category represents episodes with a mean intensity above $39 \mathrm{~m} \mathrm{~s}^{-1}$ $\left(140 \mathrm{~km} \mathrm{~h}^{-1}\right)$ approx. and duration above $36 \mathrm{~h}$ (Table A2). 


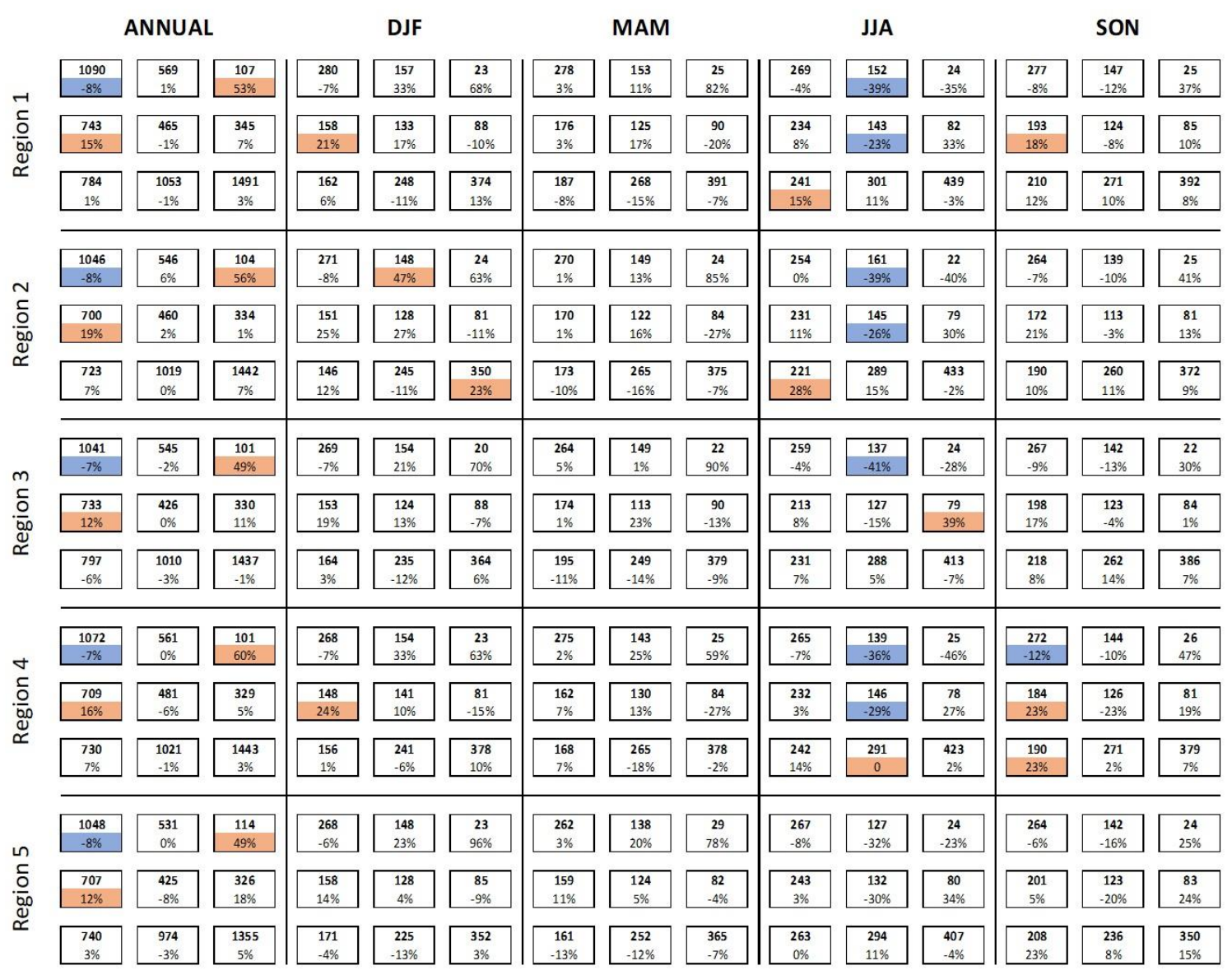

Figure 20. The number of wind speed events at $300 \mathrm{hPa}$ for each type of event, region and season. For the historical climate (1st line of the cell) and the difference between the future and historical (2nd line of the cell). Shaded values indicate significant differences ( $t$-student $5 \%$ ) positive (red) and negative (blue).

The negative statistically significant differences occur mainly for types 5, 8, and 3 (high intensity), while type 4 and 1 (short duration-less than $6 \mathrm{~h}$ ) undergoes positive differences. Summer shows the highest number of statistically significant differences (June, July and August), being mostly negative. In this case, the annual dataset does not reflect what happens in summer, but mainly in the months between December and February, where the difference between historical and future climate is mainly positive. No region stands out concerning the number of cells that are indicative of positive statistical significance. Therefore, it is generally expected that extreme events will occur in a smaller number during summer for type 8 and 3, while a higher occurrence is projected for September to February, mainly for type 4 events.

\subsubsection{Wind Shear $(0-6 \mathrm{~km})$}

Concerning the wind shear intensity (Figure 21), the majority of statistically significant values are negative differences. Autumn reveals less cells with statistically significant differences. Type 9 events (i.e., duration above $36 \mathrm{~h}$ and intensity above $25 \mathrm{~m} \mathrm{~s}^{-1}$ always have positive differences (Table A2) meaning an increase of this type of event in the future. This result is statistically significant in every region of the annual conditions, where the increase of this type of events is between $47 \%$ and $99 \%$. For winter, the expected increase of events of type 9 is between $98 \%$ and $377 \%$ and for spring months between $92 \%$ and $151 \%$. 


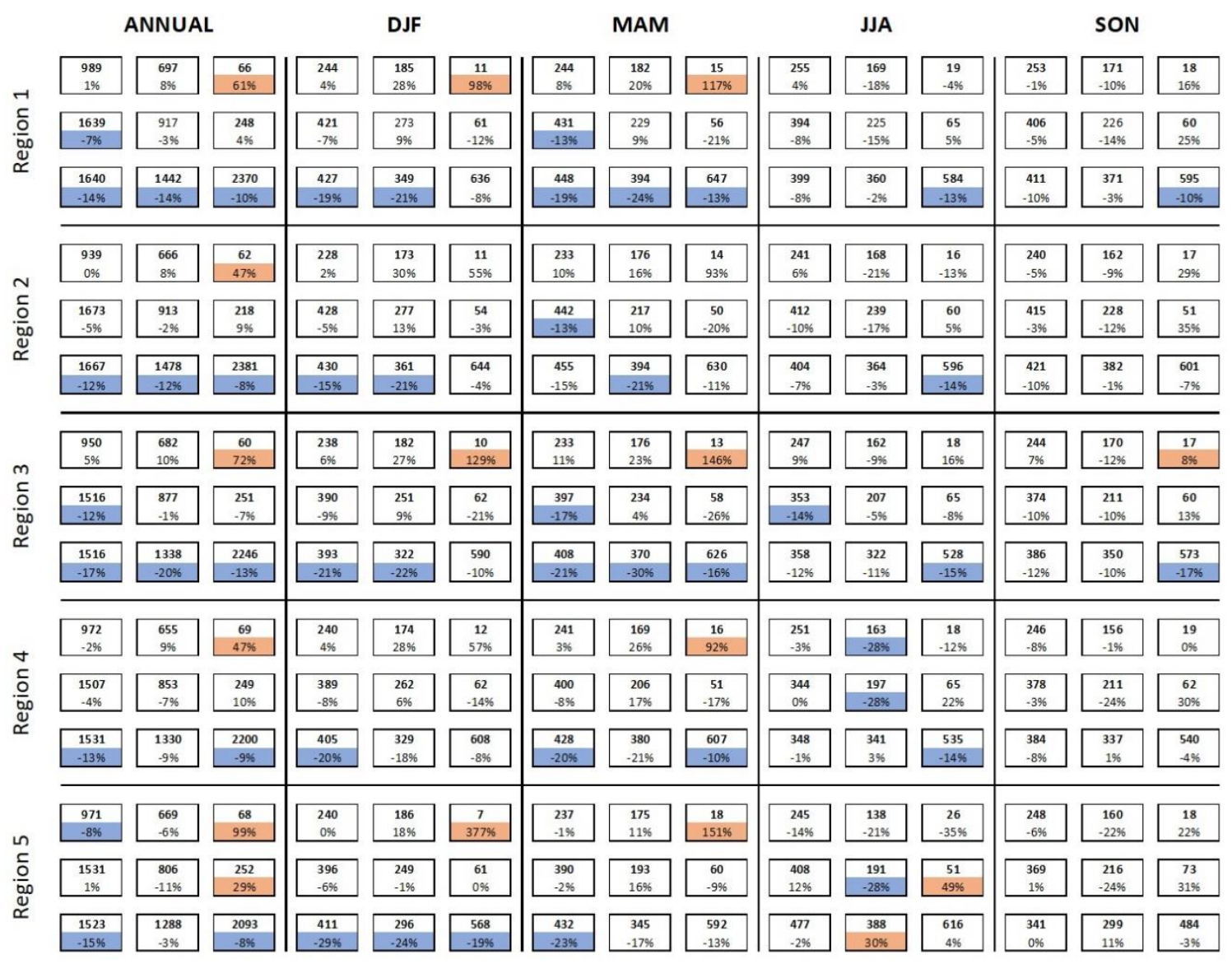

Figure 21. The number of events of the wind shear intensity between 0 and $6 \mathrm{~km}$ for each type of event, region and season. For the historical climate (1st line of the cell) and the difference between the future and historical (2nd line of the cell). Shaded values indicate significant differences (t-student $5 \%$ ) positive (red) and negative (blue).

Events with lower intensity (type 1,2 and 6-less than $8 \mathrm{~m} \mathrm{~s}^{-1}\left(28.8 \mathrm{~km} \mathrm{~h}^{-1}\right)$ approx.) are the ones presenting the majority of negative statistically significant values. For annual conditions, events of type 1 will decrease between $12 \%$ and $17 \%$, and events of type 6 between $8 \%$ and $13 \%$.

\subsubsection{Helicity $(0-3 \mathrm{~km})$}

For the helicity (Figure 22), there are many statistically significant differences between historical and future periods. There will be an overall increase in the occurrences for events with higher intensity and longer duration.

In the seasonal analysis, summer is the season that presents more positive differences. Region 3 and the summer months present the highest number of statistically significant differences. Events of type 8 (intensity above than $39 \mathrm{~m} \mathrm{~s}^{-1}\left(140 \mathrm{~km} \mathrm{~h}^{-1}\right)$ approx. (Table A2)) and 3 (intensity between $-10 \mathrm{~m}$ $\mathrm{s}^{-1}\left(-36 \mathrm{~km} \mathrm{~h}^{-1}\right)$ and $\left.39 \mathrm{~m} \mathrm{~s}^{-1}\right)$ with duration between $6 \mathrm{~h}$ and $36 \mathrm{~h}$, will tend to increase between $45-57 \%$ and $15-24 \%$, respectively. Events of type 1 and 5 (less than six hours of duration) will tend to decrease between $5 \%$ and $9 \%$ (type 1 ) and between 18\% and 26\% (type 5).

Some cells are not marked as statistically significant but present important differences. This occurs mainly for type 9 (more than $36 \mathrm{~h}$ of duration and intensity above $39 \mathrm{~m} \mathrm{~s}^{-1}\left(140 \mathrm{~km} \mathrm{~h}^{-1}\right)$ approx. (Table A2)), on region 2 during spring (317\%) and summer (300\%), and region 3 and 4 during summer ( $220 \%$ and $700 \%$, respectively). 


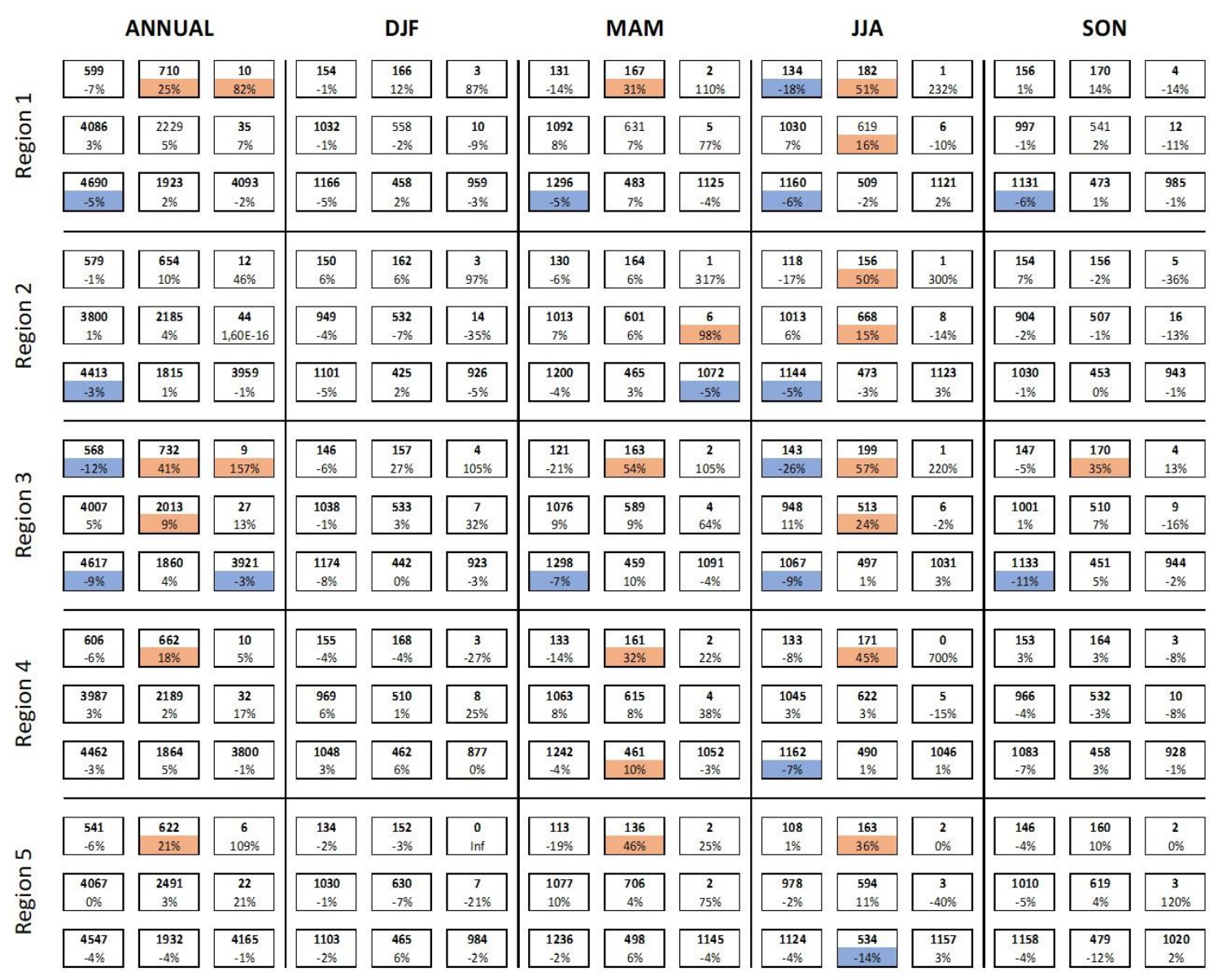

Figure 22. The number of events of helicity between 0 and $3 \mathrm{~km}$ for each type of event, region and season. For the historical climate (1st line of the cell) and the difference between the future and historical (2nd line of the cell). Shaded values indicate significant differences ( $t$-student $5 \%$ ) positive (red) and negative (blue).

\subsection{Extreme Events—Return Period}

\subsubsection{Wind at $850 \mathrm{hPa}$}

The events with a 40-year return period have a higher number of events per year and longer duration relative to the events with a 10-year return period (Figure 23a,b, respectivelly). In general, in all cities, there is a decrease in the number of events per year for both return periods (10 and 40 years), except for Bilbao. Regarding the duration of the events (days/year), it is shown that, in the future period, the events will have a shorter duration (Figure 23a).

\subsubsection{Wind at $300 \mathrm{hPa}$}

The events with a 40-year return period have a higher number of events per year relative to the 10-year return period, which means that events with a 40-year return period will be more frequent in future climates (Figure 24b). Comparing the historical and future values for both cases, there is no relation between cities. In Viseu, Coimbra, Castelo Branco, Leiria, Santarém, Portalegre, Évora, Beja, Madrid, Valencia, and Murcia, it is expected a decrease in the number of events per year for the 10-year return period events. For the 40-year return period events, the same cities remain plus Guarda and Lisboa. The remaining cities present an increase in the number of events per year. For the duration of the events, once again, the 40-year return period events are more frequent for both historical and future climates (Figure 24a). It is expected an increase for most cities, except for Évora, Beja, Faro, and Seville. 


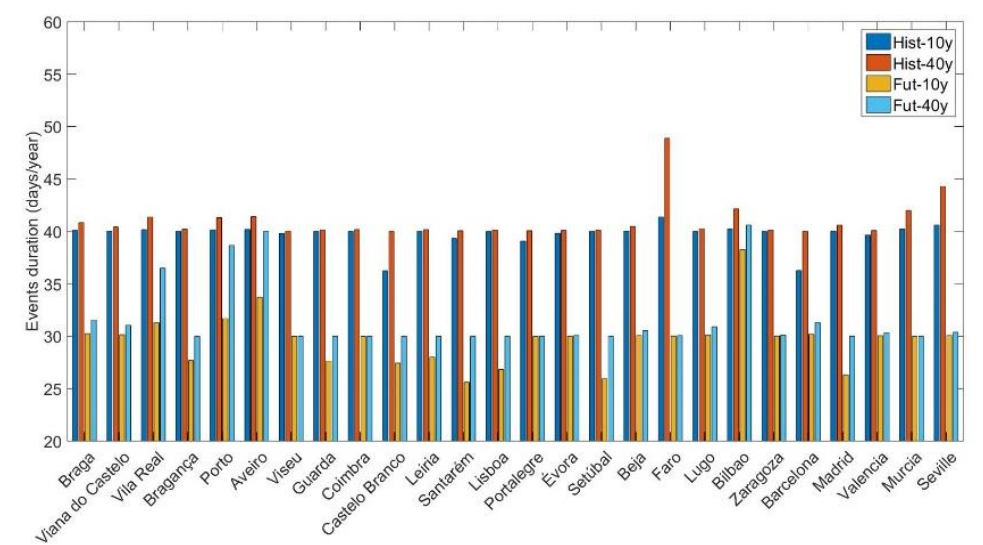

(a)

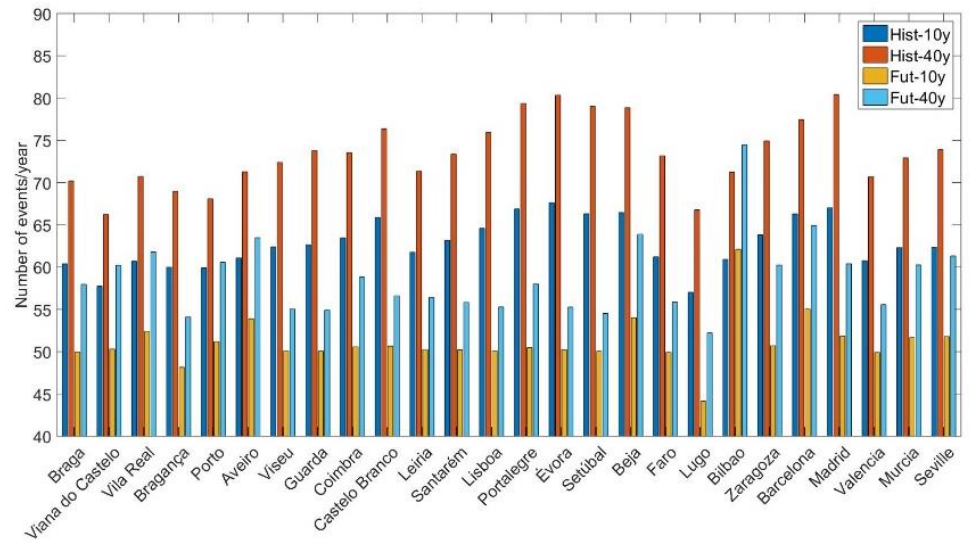

(b)

Figure 23. Comparison of the (a) event duration (days/year) and (b) the number of events (year) in the historical (blue) and future (red) period for extreme events with a return period of 10 years (full line) and 40 years (dashed line) for wind speed at $850 \mathrm{hPa}$.

\subsubsection{Wind Shear $(0-6 \mathrm{~km})$}

For the majority of the cities, the duration of extreme events of wind shear will be longer in the future, apart from Braga and Bilbao (Figure 25a). A slight difference between events with different return periods is apparent. The events with a 40-year return period are longer in some cities, but they will be lengthier than the events with a 10-year return period in most cases in the future. For the number of events per year, similar findings were obtained as for the duration of the events, but, in this case, with a stronger difference between future and historical climate and between events with 10- and 40-year return periods (Figure 25b).

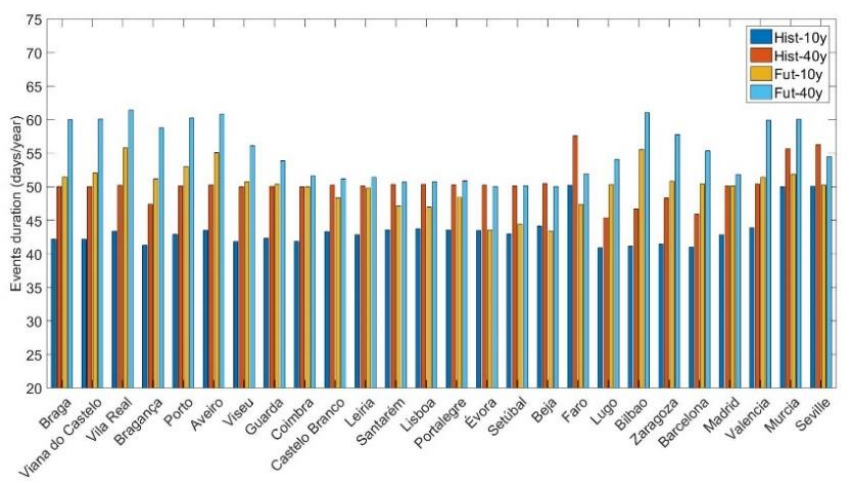

(a)

Figure 24. Cont. 


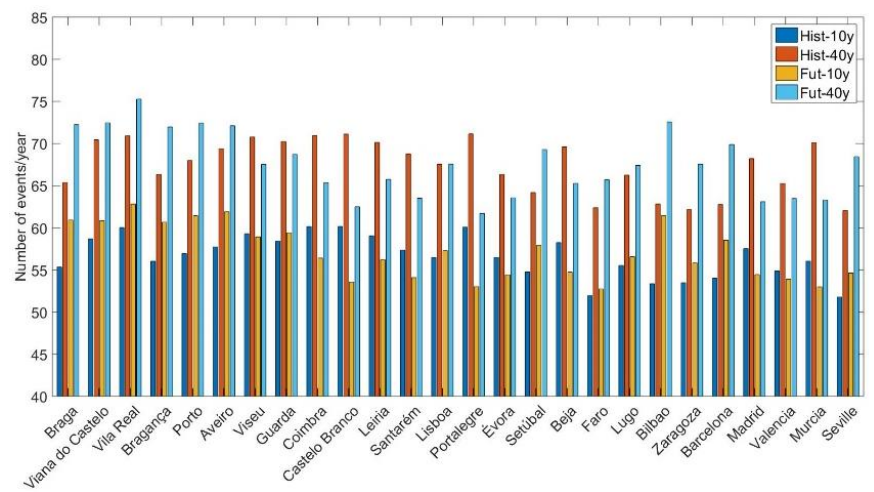

(b)

Figure 24. Comparison of the (a) event duration (days/year) and (b) the number of events (year) in the historical (blue) and future (red) period for extreme events with a return period of 10 years (full line) and 40 years (dashed line) for wind speed at $300 \mathrm{hPa}$.

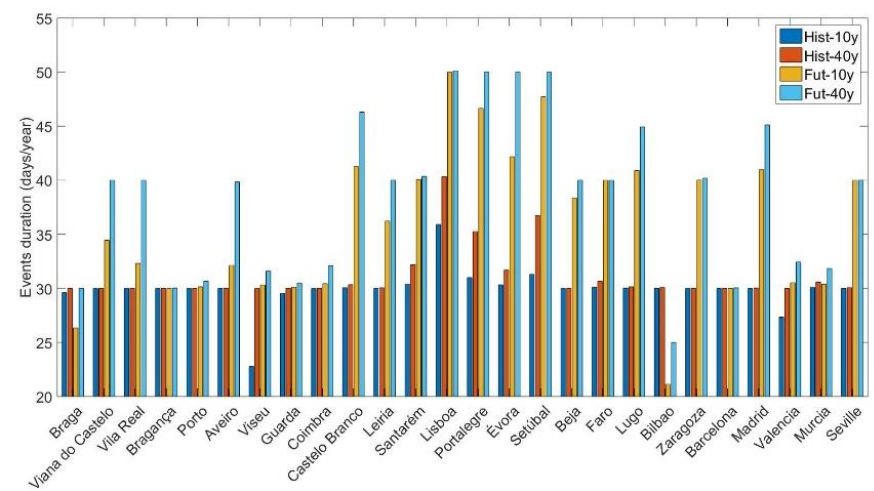

(a)

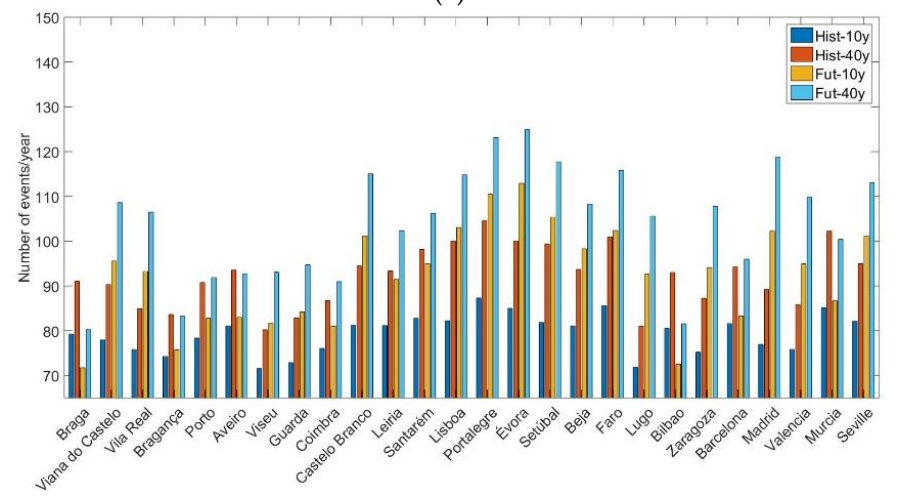

(b)

Figure 25. Comparison of the (a) event duration (days/year) and (b) the number of events (year) in the historical (blue) and future (red) period for extreme events with a return period of 10 years (full line) and 40 years (dashed line) for wind shear $(0-3 \mathrm{~km})$.

\subsubsection{Helicity $(0-3 \mathrm{~km})$}

For helicity, it is expected an increase in the duration of the events for the future (Figure 26a). It is clear that events with a return period of 40 years are longer and this difference is more evident in the future climate. Regarding the number of events per year, the same pattern is verified (Figure 26b). More events are expected in the future, except in Faro and Seville, and the events with a return period of 40 years are, and will be, more frequent than events with a 10-year return period. 


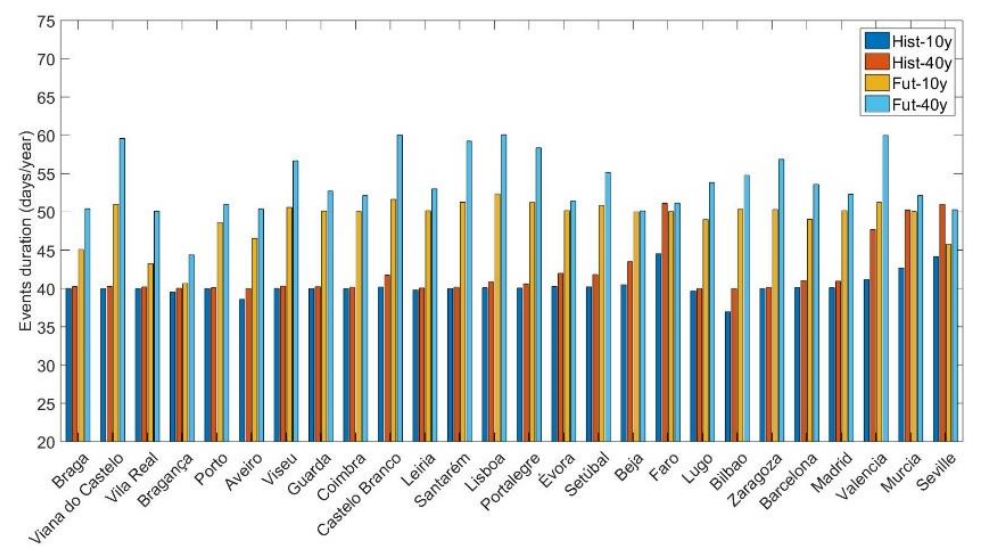

(a)

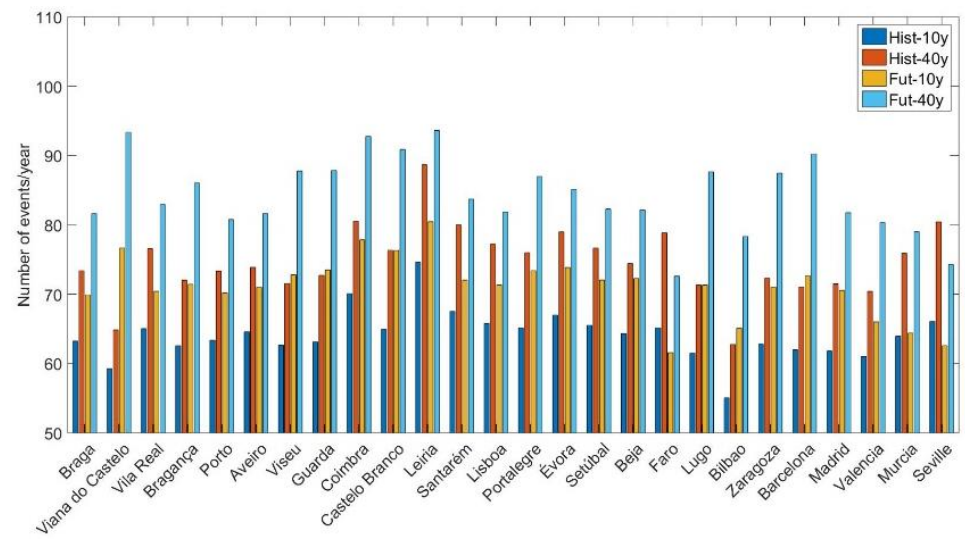

(b)

Figure 26. Comparison of the (a) event duration (days/year) and (b) the number of events (year) in the historical (blue) and future (red) period for extreme events with a return period of 10 years (full line) and 40 years (dashed line) for helicity $(0-6 \mathrm{~km})$.

\section{Discussion and Conclusions}

This study aimed to compare the historical and future climates concerning the wind speed near the surface, at $850 \mathrm{hPa}$ and $300 \mathrm{hPa}$, as well as the wind shear intensity and helicity. Although it only covers a relatively small number of cities in the Iberian Peninsula (26), it reveals some noteworthy modifications in these variables under a climate change scenario (RCP8.5). Based on the projections for a long-term future period (2080-2099), it is possible to conclude that a decrease in mean near-surface wind speed should be expected throughout most of the peninsula. Similar considerations can be made for the wind speed at $850 \mathrm{hPa}$ (decrease of approx. $5 \%$, i.e., approximately $5 \mathrm{~m} \mathrm{~s}^{-1}$ ). Furthermore, the duration and number of extreme events are projected to decrease, particularly during summer, while the corresponding return periods are expected to consistently increase. A decrease in the mean wind speed at $300 \mathrm{hPa}$ is also projected, mostly during summer. For the lower quantiles, it is expected a decrease of $4 \%\left(0.6 \mathrm{~m} \mathrm{~s}^{-1}\right)$, while an increase of $7 \%\left(4 \mathrm{~m} \mathrm{~s}^{-1}\right)$ is projected for the upper quantiles (above the median). The number and duration of extreme events will increase for events with shorter duration during winter, whereas a decrease in extreme events with high intensity is projected for summer. The return period for extreme events is projected to decrease, apart from a few exceptions. Furthermore, it is estimated that the mean wind shear intensity, duration, and extreme events (longer duration and higher intensity) will tend to increase, while extreme events with low intensity will tend to decrease. Regarding the quantiles, there is a large difference between cities. However, on average, for the superior quantiles, the difference is of approximately $5 \%\left(1.5 \mathrm{~m} \mathrm{~s}^{-1}\right)$. Accordingly, it is expected a decrease in the return period for extreme events. Lastly, regarding the helicity, it is expected a strong increase that can reach $700 \%$ with respect to the average helicity in the historical 
climate. For the extremely low quantiles, it is expected a decrease of $10 \%$, whereas, for the extremely high quantiles, an increase of $10 \%$ is projected. All types of extreme events (but, particularly, the most extreme) are projected to occur more frequently and with longer duration, mainly during summer and in the southern cities. In most cases, the return period for extreme events will probably decrease.

Many studies have revealed a decrease in the intensity of the wind at the surface in several regions, such as France [12], Czech Republic [13], Holland [14], Turkey [15], Spain, and Portugal [16]. Previous studies identified a northward displacement of the jet stream during winter, showing an increase in its intensity [23,69]. In the summer months, due to the warming of the Arctic and the weakening of the polar vortex, it is observed an equatorward displacement of the westerly jet, accompanied by a decrease in its intensity and an increase in swell. A study that was developed by [48] reveals that projections for the 21st century indicate a robust increase in the cutting wind between June and November for the tropical region of the Atlantic Ocean and East Pacific. Changes in the cutting wind over the Atlantic Ocean are related to changes in the zonal wind in the upper troposphere.

Overall, these projections will certainly have an important impact on two major socioeconomic sectors: wind energy generation and civil aviation, mainly at take-off and landing, but also on the planning of aviation routes and flight times, especially on transatlantic flights. This study also contributes to improving our knowledge on the future tornadic conditions over the Iberian Peninsula. However, this deserves further research, mainly by adding more climate model projections and anthropogenic radiative forcing scenarios, so as to increase the robustness of the present study outcomes. Climate change projection for wind are of foremost relevance for many other sectors, providing guidelines to adapt and reduce the vulnerability to climate change risks.

Author Contributions: Conceptualization, J.M., A.R., C.V., S.C.P. and J.A.S.; methodology, J.M. and A.R.; software, J.M., C.V. and S.C.P.; validation, J.M., A.R., C.V., S.C.P. and J.A.S.; formal analysis, J.M. and A.R.; investigation, J.M. and A.R.; resources, J.M., A.R., C.V., S.C.P.; data curation, J.M. and A.R.; writing-original draft preparation, J.M., A.R. and J.A.S.; writing-review and editing, J.M., A.R. and J.A.S.; visualization, J.M. and A.R.; supervision, J.M. and A.R.; project administration, J.M. and A.R.; funding acquisition, A.R. All authors have read and agreed to the published version of the manuscript.

Funding: This research was partially funded by FCT/MCTES through financial support to CESAM (UIDB/50017/2020+UIDP/50017/2020) and was was also supported by FCT-Portuguese Foundation for Science and Technology, under the project UIDB/04033/2020.

Conflicts of Interest: The authors declare no conflict of interest. The funders had no role in the design of the study; in the collection, analyses, or interpretation of data; in the writing of the manuscript, or in the decision to publish the results.

\section{Appendix A}

Table A1. List of approximate altitude (m) and pressure (hPa) for each eta level.

\begin{tabular}{ccc}
\hline Level & Altitude (m) & Pressure (hPa) \\
\hline 1 & 27 & 1009 \\
2 & 94 & 1001 \\
3 & 187 & 990 \\
4 & 305 & 977 \\
5 & 454 & 959 \\
6 & 644 & 938 \\
7 & 876 & 912 \\
8 & 1221 & 874 \\
9 & 1668 & 828 \\
10 & 2137 & 781 \\
\hline
\end{tabular}


Table A1. Cont.

\begin{tabular}{ccc}
\hline Level & Altitude (m) & Pressure (hPa) \\
\hline 11 & 2630 & 734 \\
12 & 3354 & 670 \\
13 & 4293 & 593 \\
14 & 5227 & 524 \\
15 & 6157 & 461 \\
16 & 7080 & 406 \\
17 & 7997 & 356 \\
18 & 8910 & 311 \\
19 & 9823 & 271 \\
\hline
\end{tabular}

Table A2. Table of percentile 25 and 90 based on the annual mean intensity values for wind speed at 850 and $300 \mathrm{hPa}$, wind shear (0-6) and SREH (0-3).

\begin{tabular}{|c|c|c|c|c|c|c|c|c|c|}
\hline \multirow[b]{2}{*}{ Region } & \multirow[b]{2}{*}{ Cities } & \multicolumn{2}{|c|}{ Wind at $850 \mathrm{hPa}$} & \multicolumn{2}{|c|}{ Wind at $300 \mathrm{hPa}$} & \multicolumn{2}{|c|}{ Wind Shear 0-6 } & \multicolumn{2}{|c|}{ SREH 0-3 } \\
\hline & & p25 & p90 & p25 & p90 & p25 & p90 & p25 & p90 \\
\hline \multirow{10}{*}{2} & Braga & 5.16 & 16.95 & 13.59 & 39.55 & 8.38 & 25.42 & -12.28 & 33.03 \\
\hline & Viana do Castelo & 5.03 & 17.57 & 13.45 & 39.62 & 8.28 & 24.04 & -9.01 & 41.84 \\
\hline & Vila Real & 5.49 & 16.89 & 13.75 & 39.37 & 8.53 & 25.53 & -12.40 & 36.06 \\
\hline & Bragança & 4.76 & 15.83 & 13.35 & 39.48 & 8.76 & 27.10 & -12.24 & 64.64 \\
\hline & Porto & 5.18 & 16.88 & 13.74 & 39.24 & 8.12 & 24.59 & -11.92 & 34.37 \\
\hline & Aveiro & 5.28 & 17.50 & 13.93 & 38.85 & 7.79 & 23.30 & -11.96 & 31.63 \\
\hline & Viseu & 4.26 & 14.38 & 13.49 & 39.77 & 9.27 & 26.01 & -11.12 & 43.64 \\
\hline & Guarda & 4.38 & 14.55 & 13.30 & 39.00 & 9.04 & 25.43 & -12.43 & 28.16 \\
\hline & Coimbra & 4.20 & 13.91 & 13.34 & 39.38 & 9.15 & 25.04 & -15.96 & 31.00 \\
\hline & Lugo & 5.29 & 18.55 & 13.49 & 40.48 & 9.34 & 27.56 & -5.11 & 71.19 \\
\hline \multirow{10}{*}{3} & Castelo Branco & 4.15 & 13.41 & 13.47 & 38.98 & 9.56 & 25.04 & -8.07 & 42.48 \\
\hline & Leiria & 4.29 & 13.99 & 13.45 & 39.22 & 8.77 & 24.72 & -14.53 & 40.35 \\
\hline & Santarém & 4.24 & 13.52 & 13.57 & 38.79 & 9.40 & 24.30 & -9.18 & 39.56 \\
\hline & Lisboa & 4.31 & 13.85 & 13.69 & 38.78 & 9.59 & 24.36 & -7.78 & 44.08 \\
\hline & Portalegre & 4.16 & 14.15 & 13.71 & 38.70 & 9.60 & 25.01 & -5.44 & 42.90 \\
\hline & Évora & 4.09 & 14.53 & 13.84 & 38.75 & 9.65 & 24.97 & -7.88 & 42.52 \\
\hline & Setúbal & 4.23 & 14.13 & 13.68 & 38.74 & 9.64 & 24.56 & -8.35 & 40.90 \\
\hline & Beja & 3.99 & 14.86 & 13.94 & 38.94 & 9.39 & 24.80 & -11.23 & 33.40 \\
\hline & Faro & 4.09 & 13.20 & 11.68 & 36.93 & 7.56 & 21.35 & -7.20 & 26.52 \\
\hline & Seville & 3.87 & 13.04 & 11.92 & 37.14 & 7.58 & 22.19 & -5.64 & 31.86 \\
\hline \multirow{4}{*}{4} & Bilbao & 5.35 & 16.48 & 13.70 & 40.10 & 8.96 & 27.68 & -10.67 & 52.63 \\
\hline & Zaragoza & 4.98 & 16.51 & 13.22 & 40.33 & 8.11 & 24.38 & -9.92 & 26.24 \\
\hline & Barcelona & 3.81 & 14.85 & 12.97 & 40.04 & 9.82 & 27.43 & -15.63 & 46.54 \\
\hline & Madrid & 4.41 & 14.45 & 13.68 & 38.87 & 9.36 & 25.76 & -6.80 & 36.54 \\
\hline \multirow{2}{*}{5} & Valencia & 3.61 & 14.95 & 12.70 & 38.54 & 9.12 & 25.40 & -15.56 & 33.04 \\
\hline & Murcia & 3.79 & 13.62 & 12.68 & 38.01 & 8.35 & 24.39 & -16.04 & 30.90 \\
\hline
\end{tabular}

\section{References}

1. McVicar, T.R.; Van Niel, T.G.; Li, L.T.; Roderick, M.L.; Rayner, D.P.; Ricciardulli, L.; Donohue, R.J. Wind speed climatology and trends for Australia, 1975-2006: Capturing the stilling phenomenon and comparison with near-surface reanalysis output. Geophys. Res. Lett. 2008, 35. [CrossRef]

2. Fernandes, P.M.; Barros, A.M.G.; Pinto, A.; Santos, J.A. Characteristics and controls of extremely large wildfires in the western Mediterranean Basin. J. Geophys. Res. Biogeosci. 2016, 121, 2141-2157. [CrossRef]

3. McInnes, K.L.; Macadam, I.; Hubbert, G.D.; O'Grady, J.G. A modelling approach for estimating the frequency of sea level extremes and the impact of climate change in southeast Australia. Nat. Hazards 2009, 51, 115-137. [CrossRef]

4. Pirazzoli, P.A.; Tomasin, A. Recent near-surface wind changes in the central Mediterranean and Adriatic areas. Int. J. Climatol. 2003, 23, 963-973. [CrossRef]

5. Nechaj, P.; Gaál, L.; Bartok, J.; Vorobyeva, O.; Gera, M.; Kelemen, M.; Polishchuk, V. Monitoring of low-level wind shear by ground-based 3D lidar for increased flight safety, protection of human lives and health. Int. J. Environ. Res. Public Health 2019, 16, 4584. [CrossRef] 
6. Santos, J.A.; Woollings, T.; Pinto, J.G. Are the winters 2010 and 2012 archetypes exhibiting extreme opposite behavior of the north atlantic jet stream. Mon. Weather Rev. 2013, 141, 3626-3640. [CrossRef]

7. Woollings, T.; Pinto, J.G.; Santos, J.A. Dynamical evolution of North Atlantic ridges and Poleward Jet stream displacements. J. Atmos. Sci. 2011, 68, 954-963. [CrossRef]

8. Santos, J.; Pinto, J.; Ulbrich, U. On the development of strong ridge episodes over the eastern North Atlantic. Geophys. Res. Lett. 2009, 36. [CrossRef]

9. Andrade, C.; Santos, J.; Pinto, J.; Corte-Real, J. Large-scale atmospheric dynamics of the wet winter 2009-2010 and its impact on hydrology in Portugal. Clim. Res. 2011, 46, 29-41. [CrossRef]

10. Santos, J.A.; Rochinha, C.; Liberato, M.L.R.; Reyers, M.; Pinto, J.G. Projected changes in wind energy potentials over Iberia. Renew. Energy 2015, 75, 68-80. [CrossRef]

11. Bichet, A.; Wild, M.; Folini, D.; Schr, C. Causes for decadal variations of wind speed over land: Sensitivity studies with a global climate model. Geophys. Res. Lett. 2012, 39, 4-9. [CrossRef]

12. Najac, J.; Boé, J.; Terray, L. A multi-model ensemble approach for assessment of climate change impact on surface winds in France. Clim. Dyn. 2009, 32, 615-634. [CrossRef]

13. Brázdil, R.; Chromá, K.; Dobrovolny, P.; Tolasz, R. Climate fluctuations in the Czech Republic during the period 1961-2005. Int. J. Climatol. 2009, 29, 223-242. [CrossRef]

14. Cusack, S. A 101 year record of windstorms in the Netherlands. Clim. Chang. 2013, 116, 693-704. [CrossRef]

15. Dadaser-Celik, F.; Cengiz, E. Wind speed trends over Turkey from 1975 to 2006. Int. J. Climatol. 2014, 34, 1913-1927. [CrossRef]

16. Vicente-Serrano, S.M.; Lopez-Moreno, J.I.; Beguería, S.; Lorenzo-Lacruz, J.; Sanchez-Lorenzo, A.; García-Ruiz, J.M.; Azorin-Molina, C.; Morán-Tejeda, E.; Revuelto, J.; Trigo, R.; et al. Evidence of increasing drought severity caused by temperature rise in southern Europe. Environ. Res. Lett. 2014, 9, 044001. [CrossRef]

17. Holt, E.; Wang, J. Trends in wind speed at wind turbine height of $80 \mathrm{~m}$ over the contiguous United States using the north American Regional Reanalysis (NARR). J. Appl. Meteorol. Climatol. 2012, 51, 2188-2202. [CrossRef]

18. Earl, N.; Dorling, S.; Hewston, R.; Von Glasow, R. 2010-1980 Variability in U.K. surface wind climate. J. Clim. 2013, 26, 1172-1191. [CrossRef]

19. Jerez, S.; Trigo, R.M.; Vicente-Serrano, S.M.; Pozo-VáZquez, D.; Lorente-Plazas, R.; Lorenzo-Lacruz, J.; Santos-Alamillos, F.; MontáVez, J.P. The impact of the north atlantic oscillation on renewable energy resources in Southwestern Europe. J. Appl. Meteorol. Climatol. 2013, 52, 2204-2225. [CrossRef]

20. Wan, H.; Wang, X.L.; Swail, V.R. Homogenization and trend analysis of Canadian near-surface wind speeds. J. Clim. 2010, 23, 1209-1225. [CrossRef]

21. Carvalho, D.; Rocha, A.; Gómez-Gesteira, M.; Silva Santos, C. Potential impacts of climate change on European wind energy resource under the CMIP5 future climate projections. Renew. Energy 2017, 101, $29-40$. [CrossRef]

22. Irvine, E.A.; Shine, K.P.; Stringer, M.A. What are the implications of climate change for trans-Atlantic aircraft routing and flight time? Transp. Res. Part D Transp. Environ. 2016, 47, 44-53. [CrossRef]

23. Peings, Y.; Cattiaux, J.; Vavrus, S.; Magnusdottir, G. Late twenty-first-century changes in the midlatitude atmospheric circulation in the CESM large ensemble. J. Clim. 2017, 30, 5943-5960. [CrossRef]

24. IPCC. Managing the Risks of Extreme Events and Disasters to Advance Climate Change Adaptation: A Special Report of Working Groups I and II of the Intergovernmental Panel on Climate Change; Cambridge University Press: Cambridge, UK, 2012; ISBN 9781107607804.

25. Donat, M.G.; Leckebusch, G.C.; Pinto, J.G.; Ulbrich, U. Examination of wind storms over Central Europe with respect to circulation weather types and NAO phases. Int. J. Climatol. 2010, 30, 1289-1300. [CrossRef]

26. McBean, G. An integrated approach to air pollution, climate and weather hazards. Policy Options 2006, 27, $18-24$.

27. Doswell, C.A.; Evans, J.S. Proximity sounding analysis for derechos and supercells: An assessment of similarities and differences. Atmos. Res. 2003, 67-68, 117-133. [CrossRef]

28. Thompson, R.L.; Edwards, R.; Hart, J.A.; Elmore, K.L.; Markowski, P. Close proximity soundings within supercell environments obtained from the rapid update cycle. Weather Forecast. 2003, 18, 1243-1261. [CrossRef] 
29. Groenemeijer, P.H.; van Delden, A. Sounding-derived parameters associated with large hail and tornadoes in the Netherlands. Atmos. Res. 2007, 83, 473-487. [CrossRef]

30. Rodríguez, O.; Bech, J. Sounding-derived parameters associated with tornadic storms in Catalonia. Int. J. Climatol. 2018, 38, 2400-2414. [CrossRef]

31. Rasmussen, E.N.; Blanchard, D.O. A baseline climatology of sounding-derived supercell and tornado forecast parameters. Weather Forecast. 1998, 13, 1148-1164. [CrossRef]

32. Antonescu, B.; Schultz, D.M.; Holzer, A.; Groenemeijer, P. Tornadoes in Europe: An underestimated threat. Bull. Am. Meteorol. Soc. 2017, 98, 713-728. [CrossRef]

33. Antonescu, B.; Schultz, D.M.; Lomas, F.; Kühne, T. Tornadoes in Europe: Synthesis of the observational datasets. Mon. Weather Rev. 2016, 144, 2445-2480. [CrossRef]

34. Gayà, M. Els Fiblons a Espanya: Climatologia i Catàleg de Tornados i Trombes; Edicions UIB: Palma, Spain, 2015; ISBN 978-84-8384-315-4.

35. Gayà, M.; Llasat, M.C.; Arús, J. Erratum: Tornadoes and waterspouts in Catalonia (1950-2009) (Natural Hazards and Earth System Science (2011) 11 (1875-1883)). Nat. Hazards Earth Syst. Sci. 2011, 11, 3023. [CrossRef]

36. Riesco Martín, J.; Polvorinos Pascual, F.; Núñez Mora, J.Á.; Soriano Romero, J.D.; Jiménez Alonso, C. Climatología de Tornados en España Peninsular y Baleares; Agencia Estatal de Meteorología: Madrid, Spain, 2015.

37. Doswell III, C.; Schultz, D. On the use of indices and parameters in forecasting. Electron. J. Sev. Storms Meteorol. 2006, 1, 1-22.

38. Rauhala, J.; Schultz, D.M. Severe thunderstorm and tornado warnings in Europe. Atmos. Res. 2009, 93, 369-380. [CrossRef]

39. Miglietta, M.M.; Rotunno, R. An EF3 multivortex tornado over the ionian region: Is it time for a dedicated warning system over Italy? Bull. Am. Meteorol. Soc. 2016, 97, 337-344. [CrossRef]

40. Boldingh Debernard, J.; Petter Røed, L. Future wind, wave and storm surge climate in the Northern Seas: A revisit. Tellus Ser. A Dyn. Meteorol. Oceanogr. 2008, 60 A, 427-438. [CrossRef]

41. Donat, M.G.; Leckebusch, G.C.; Wild, S.; Ulbrich, U. Future changes in European winter storm losses and extreme wind speeds inferred from GCM and RCM multi-model simulations. Nat. Hazards Earth Syst. Sci. 2011, 11, 1351-1370. [CrossRef]

42. Donat, M.G.; Leckebusch, G.C.; Pinto, J.G.; Ulbrich, U. European storminess and associated circulation weather types: Future changes deduced from a multi-model ensemble of GCM simulations. Clim. Res. 2010, 42, 27-43. [CrossRef]

43. Pinto, J.G.; Fröhlich, E.L.; Leckebusch, G.C.; Ulbrich, U. Changing European storm loss potentials under modified climate conditions according to ensemble simulations of the ECHAM5/MPI-OM1 GCM. Nat. Hazards Earth Syst. Sci. 2007, 7, 165-175. [CrossRef]

44. Sterl, A.; Van Den Brink, H.; De Vries, H.; Haarsma, R.; Van Meijgaard, E. An ensemble study of extreme storm surge related water levels in the North Sea in a changing climate. Ocean Sci. 2009, 5, 369-378. [CrossRef]

45. Cardoso, R.M.; Soares, P.M.M.; Lima, D.C.A.; Miranda, P.M.A. Mean and extreme temperatures in a warming climate: EURO CORDEX and WRF regional climate high-resolution projections for Portugal. Clim. Dyn. 2019, 52, 129-157. [CrossRef]

46. Santos, M.; Fonseca, A.; Fragoso, M.; Santos, J.A. Recent and future changes of precipitation extremes in mainland Portugal. Theor. Appl. Climatol. 2019, 137, 1305-1319. [CrossRef]

47. Soares, P.M.M.; Cardoso, R.M.; Lima, D.C.A.; Miranda, P.M.A. Future precipitation in Portugal: High-resolution projections using WRF model and EURO-CORDEX multi-model ensembles. Clim. Dyn. 2017, 49, 2503-2530. [CrossRef]

48. Vecchi, G.A.; Soden, B.J. Increased tropical Atlantic wind shear in model projections of global warming. Geophys. Res. Lett. 2007, 34, 1-5. [CrossRef]

49. Viceto, C.; Marta-Almeida, M.; Rocha, A. Future climate change of stability indices for the Iberian Peninsula. Int. J. Climatol. 2017, 37, 4390-4408. [CrossRef]

50. Marsh, P.T.; Brooks, H.E.; Karoly, D.J. Preliminary investigation into the severe thunderstorm environment of Europe simulated by the Community Climate System Model 3. Atmos. Res. 2009, 93, 607-618. [CrossRef]

51. Mcinnes, K.L.; Erwin, T.A.; Bathols, J.M. Global Climate Model projected changes in $10 \mathrm{~m}$ wind speed and direction due to anthropogenic climate change. Atmos. Sci. Lett. 2011, 12, 325-333. [CrossRef] 
52. Marta-Almeida, M.; Teixeira, J.C.; Carvalho, M.J.; Melo-Gonçalves, P.; Rocha, A.M. High resolution WRF climatic simulations for the Iberian Peninsula: Model validation. Phys. Chem. Earth 2016, 94, 94-105. [CrossRef]

53. Dee, D.P.; Uppala, S.M.; Simmons, A.J.; Berrisford, P.; Poli, P.; Kobayashi, S.; Andrae, U.; Balmaseda, M.A.; Balsamo, G.; Bauer, P.; et al. The ERA-Interim reanalysis: Configuration and performance of the data assimilation system. Q. J. R. Meteorol. Soc. 2011, 137, 553-597. [CrossRef]

54. Giorgetta, M.A.; Jungclaus, J.; Reick, C.H.; Legutke, S.; Bader, J.; Böttinger, M.; Brovkin, V.; Crueger, T.; Esch, M.; Fieg, K.; et al. Climate and carbon cycle changes from 1850 to 2100 in MPI-ESM simulations for the Coupled Model Intercomparison Project phase 5. J. Adv. Model. Earth Syst. 2013, 5, 572-597. [CrossRef]

55. Sillmann, J.; Kharin, V.V.; Zwiers, F.W.; Zhang, X.; Bronaugh, D. Climate extremes indices in the CMIP5 multimodel ensemble: Part 2. Future climate projections. J. Geophys. Res. Atmos. 2013, 118, 2473-2493. [CrossRef]

56. Stocker, T.F.; Dahe, Q.; Plattner, G.-K.; Alexander, L.V.; Allen, S.K.; Bindoff, N.L.; Bréon, F.-M.; Church, J.A.; Cubash, U.; Emori, S.; et al. IPCC Technical Summary AR5; Climate Change 2013 Physical Science Basis. Contribution of Working Group I to Fifth Assessment Report of the Intergovernmental Panel on Climate Change; IPCC: Geneva, Switzerland, 2013; pp. 33-115.

57. Amengual, A.; Homar, V.; Romero, R.; Alonso, S.; Ramis, C. A statistical adjustment of regional climate model outputs to local scales: Application to Platja de Palma, Spain. J. Clim. 2012, 25, 939-957. [CrossRef]

58. Viceto, C.; Pereira, S.C.; Rocha, A. Climate change projections of extreme temperatures for the Iberian Peninsula. Atmosphere 2019, 10, 229. [CrossRef]

59. Cardoso Pereira, S.; Marta-Almeida, M.; Carvalho, A.C.; Rocha, A. Extreme precipitation events under climate change in the Iberian Peninsula. Int. J. Climatol. 2020, 40, 1255-1278. [CrossRef]

60. Burgess, D.; Foster, M. Test of Helicity as a Tornado Forecast Parameter; Preprints; American Meteor Society: Kananaskis Park, AB, Canada, 1990.

61. Thompson, R.L.; Mead, C.M.; Edwards, R. Effective storm-relative helicity and bulk shear in supercell thunderstorm environments. Weather Forecast. 2007, 22, 102-115. [CrossRef]

62. Kerr, B.W.; Darkow, G.L. Storm-relative winds and helicity in tornadic thunderstorm environments. Weather Forecast. 1996, 11, 489-505. [CrossRef]

63. Lai, C.-D.; Xie, M. Weibull Distributions and Their Applications. In Springer Handbook of Engineering Statistics; Springer: Berlin/Heidelberg, Germany, 2006; pp. 63-78.

64. Forbes, C.; Evans, M.; Hastings, N.; Peacock, B. Extreme Value (Gumbel) Distribution. In Statistical Distributions, 4th ed.; John Wiley and Sons: Hoboken, NJ, USA, 2010; pp. 98-101.

65. Barnes, E.A.; Polvani, L. Response of the Midlatitude Jets, and of Their Variability, to Increased Greenhouse Gases in the CMIP5 Models. J. Clim. 2013, 26, 7117-7135. [CrossRef]

66. Butler, A.; Thompson, D.; Heikes, R. The Steady-State Atmospheric Circulation Response to Climate Change-like Thermal Forcings in a Simple General Circulation Model. J. Clim. 2010, 23, 3474-3496. [CrossRef]

67. Chen, G.; Held, I.M. Phase speed spectra and the recent poleward shift of Southern Hemisphere surface westerlies. Geophys. Res. Lett. 2007, 34. [CrossRef]

68. Harvey, B.J.; Shaffrey, L.C.; Woollings, T.J. Equator-to-pole temperature differences and the extra-tropical storm track responses of the CMIP5 climate models. Clim. Dyn. 2014, 43, 1171-1182. [CrossRef]

69. Barnes, E.A.; Simpson, I.R. Seasonal sensitivity of the Northern Hemisphere jet streams to Arctic temperatures on subseasonal time scales. J. Clim. 2017, 30, 10117-10137. [CrossRef]

(C) 2020 by the authors. Licensee MDPI, Basel, Switzerland. This article is an open access article distributed under the terms and conditions of the Creative Commons Attribution (CC BY) license (http://creativecommons.org/licenses/by/4.0/). 